\title{
WestVirginiaUniversity
}

THE RESEARCH REPOSITORY @ WVU

Graduate Theses, Dissertations, and Problem Reports

2004

\section{Super resolution of fingerprints}

Kaustubh R. Deshpande
West Virginia University

Follow this and additional works at: https://researchrepository.wvu.edu/etd

\section{Recommended Citation}

Deshpande, Kaustubh R., "Super resolution of fingerprints" (2004). Graduate Theses, Dissertations, and Problem Reports. 1485.

https://researchrepository.wvu.edu/etd/1485

This Thesis is protected by copyright and/or related rights. It has been brought to you by the The Research Repository @ WVU with permission from the rights-holder(s). You are free to use this Thesis in any way that is permitted by the copyright and related rights legislation that applies to your use. For other uses you must obtain permission from the rights-holder(s) directly, unless additional rights are indicated by a Creative Commons license in the record and/ or on the work itself. This Thesis has been accepted for inclusion in WVU Graduate Theses, Dissertations, and Problem Reports collection by an authorized administrator of The Research Repository @ WVU. For more information, please contact researchrepository@mail.wvu.edu. 


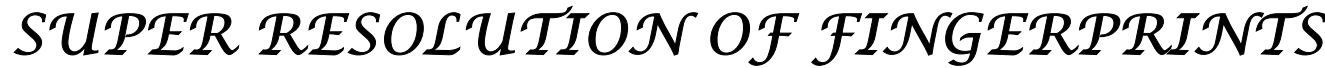

\author{
by \\ Kaustu6h RDeshpande \\ Thesis submitted to the College of Engineering and Mineral Resources \\ at West Virginia University \\ in partial fulfillment of the requirements \\ for the degree of \\ Master of Electrical Engineering \\ in \\ Communications and Signal Processing
}

Approved by

Dr. Xin Li, Committee Chairperson

Dr. Arun Ross

Dr. Natalia Schmid

Dr. Donald Adjeroh

Lane Department of Computer Science and Electrical Engineering

Morgantown, West Virginia

2004

Keywords:

Super Resolution, Biometrics, Fingerprints, Blind Deconvolution, Anisotropic Coherence Enhancement, Fingerprint Enhancement, Partial Differential Equations

Copyright 2004 Kaustubh R. Deshpande 


\section{ABSTRACT \\ SUPER RESOLUTION OF FINGERPRINTS}

\section{by Kaustubh R. Deshpande}

The goal of any biometric recognition algorithm has been to target $100 \%$ recognition rate. Even a failure of $0.1 \%$ of the cases may be disastrous to the overall performance of the algorithm given the nature of the application. The performance of the recognition module is dependent directly on the quality of the images fed to this algorithm. It is no wonder thus that enhancing these images before they are fed into the recognition algorithm has gained importance in the recent years.

In this work, we develop a super-resolution method with a specific goal to improve the resolution of biometric images. This method can be used as a preprocessing step in a variety of biometric applications including fingerprint enhancement with the purpose of detecting pores on fingerprints, compression of biometric data using distributed coding, and increasing the resolution of facial images with the purpose to improve performance of the face based recognition systems. This preprocessing step is of great importance since it admits the use of low quality of-the-shelf sensors for data collection in place of high resolution expensive sensors to capture biometric images.

The implementation of the algorithm can be divided into two steps. This can be also visualized as passing a sequence of low resolution images through a system with two sequentially connected modules. The first module uses the available multiple copies of the same subject to construct a raw high resolution image. The second module is a stand alone restoration module which enhances the raw high resolution image to provide the final refined image. The first module is a general module which does not use any information about the nature of the image for enhancing the resolution whereas the restoration module uses the a priori information about the nature of the image and enhances it accordingly.

For the registration module, we have combined the widely accepted global phase correlation method to align the multiple images together and a novel application of the projection onto the convex sets theory for interpolating these aligned images and mapping them on the high resolution pixel grid.

The restoration module is an amalgam of three different processes viz. blind deconvolution, coherence based anisotropic diffusion and the image inpainting which stand alone by themselves as efficient and productive means of image enhancement. The blind deconvolution technique does not assume any knowledge of the blurring kernel and deblurrs the image effectively. The coherence based anisotropic diffusion is a technique for smoothing which takes into account geometry of the image along with its contrast and thus is effective for fingerprints. The image inpainting technique on the other hand tries to fill up small discontinuities such as holes or scars on the fingerprint and thus, adds texture information in the image. 


\section{ACKNOWLEDGMENTS}

A few lines of acknowledgement do not fully express my gratitude and appreciation for those who guided and supported me through these last two years. I have been fortunate to be surrounded by teachers, family and friends with whom I can share all my joy and frustrations.

I have been privileged to have Dr. Xin Li as my advisor. His attention to detail, quest for excellence, love for perfection, ingenious ideas and understanding has always motivated me to give my best time and again. He has helped me develop and tune my academic skills including research, presentation and writing skills. I am greatly indebted to him for making my masters experience a memorable one.

I am grateful to Dr. Donald Adjeroh, Dr. Arun Ross and Dr. Natalia Schmid for their expert guidance and understanding both in academics as well as in my personal life. When faced with difficulties and dilemmas, I always knew I could rely on them for guidance. This was a plus for me psychologically because for international students like me this is like a home away from home. I would also take this opportunity to thank Mr. Vic Baker and the team at the GVL for their support during my first year at WVU. I would also like to thank Dr. Lixin Shen and Dr. Matthew Valenti for giving me a chance to study under their guidance.

I would also like to thank my lab mates at the Image and Video Coding Lab for their support and interactive technical discussions which often gave me ideas for my research. I wish all of them success in their future endeavors. I thank my friends for their continuing support both from India, as well as from the United States.

A special mention has to be made of all the professors at WVU for creating a conducive environment for studying and conducting quality research. This would not have been possible without the help of the department secretaries and the systems group. My heartfelt thanks to them for their excellent work and support.

Finally, I would like to thank my grandparents, my parents, my brother and my relatives for their support, efforts and sacrifice without which I would not have come this far. Last but not the least I thank my girl friend, Asawari for understanding me, standing by me and sharing my joys and frustrations. I know there is a great future awaiting us.

To all of you I dedicate this work. 


\section{TABLE OF CONTENTS}

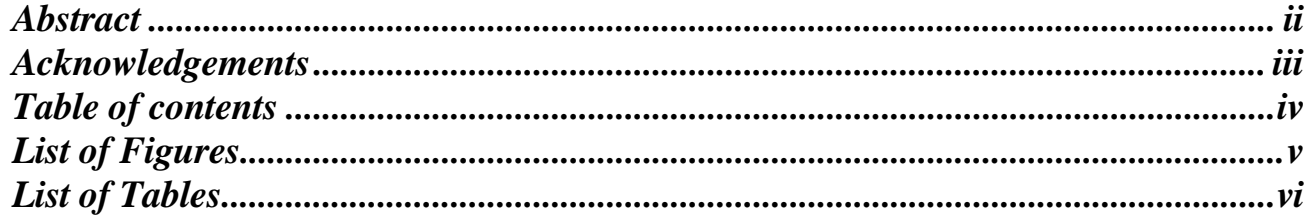

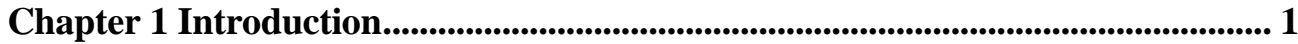

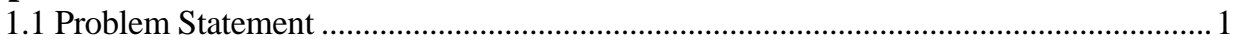

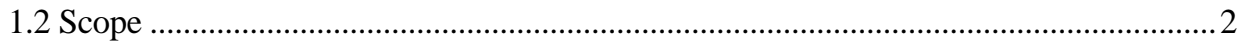

1.3 Background and Related Work .......................................................................................

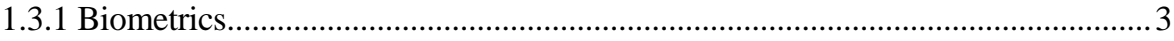

1.3.1.1 Fingerprints ...........................................................................................

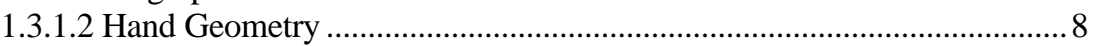

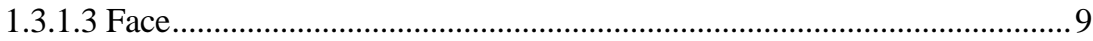

1.3.1.4 Iris ................................................................................................ 10

1.3.2 Fingerprint Enhancement ................................................................................11

1.3.3 Super Resolution .............................................................................................. 13

1.3.4 Image Alignment................................................................................................ 15

1.3.5 Image Interpolation .....................................................................................17

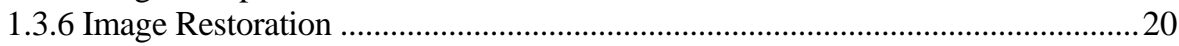

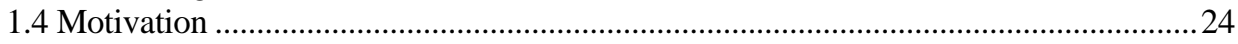

1.5 Thesis Organization....................................................................................................26

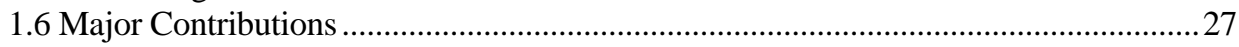

Chapter 2 Image Registration ...................................................................................... 30

2.1 Image Alignment........................................................................................................... 31

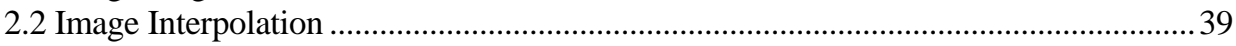

Chapter 3 Enhancement Techniques.......................................................................... 49

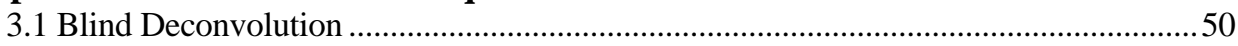

3.2 Anisotropic Coherence Enhancement.............................................................................54

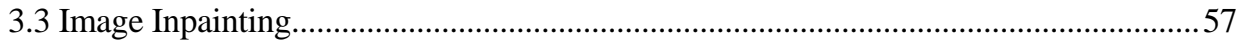

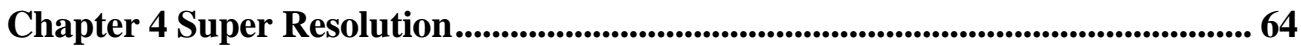

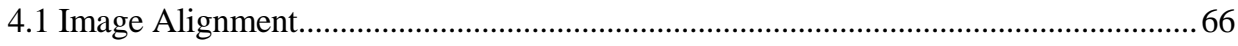

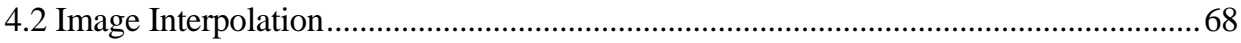

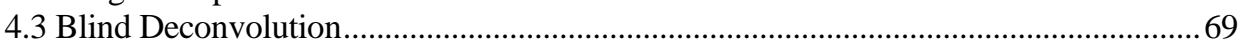

4.4 Anisotropic Coherence Enhancement ......................................................................... 70

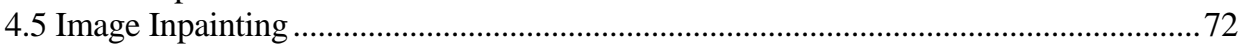

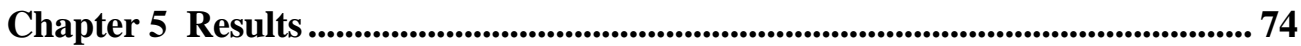

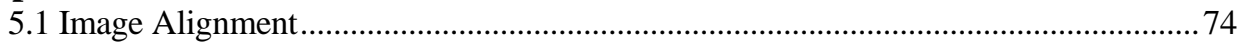

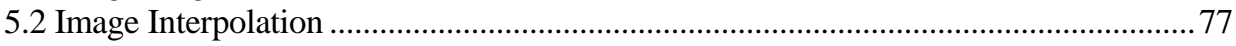

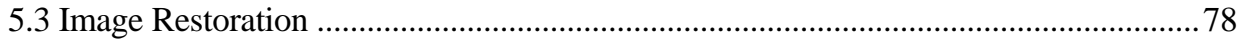

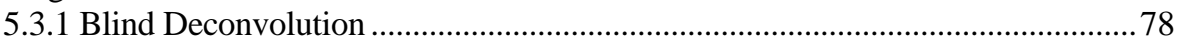

5.3.2 Anisotropic Coherence Enhancement.................................................................... 79

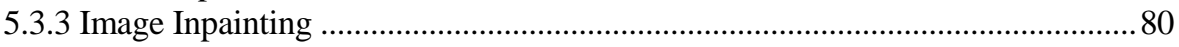

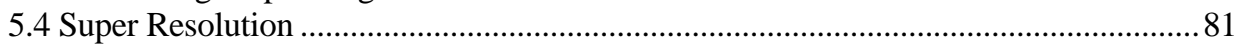

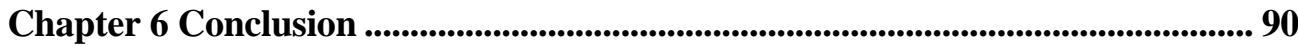

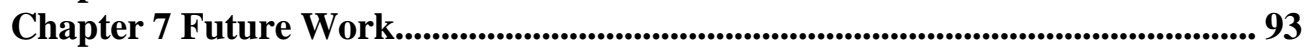

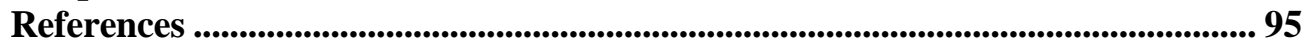




\section{LIST OF FIGURES}

Figure 1: Examples of some biometric traits associated with an individual ................ 4

Figure 2: Components of a simple biometric system ................................................. 6

Figure 3: Features of a fingerprint ……………….................................................. 7

Figure 4: Hand Geometry features and acquired image................................................ 8

Figure 5: Principal component analysis for face recognition, the eigen vector

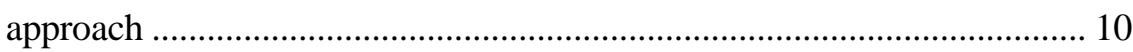

Figure 6: Original iris image and its processed version .............................................. 10

Figure 7: Images (a) and (c) are enhanced to achieve a better quality image

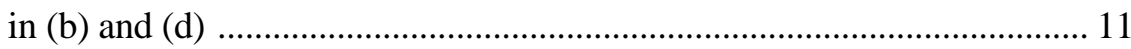

Figure 8: Image restoration results ....................................................................... 21

Figure 9: Correlation peak location for translated images only ................................. 33

Figure 10: Fingerprint images and their log polar transforms …................................ 34

Figure 11: Images with sub pixel displacements and their cross power spectra with noise and after noise removal ........................................................ 35

Figure 12: Spatial and frequency response of polyphase filter ..................................... 37

Figure 13: Flowchart for estimating the affine parameters …………………............. 38

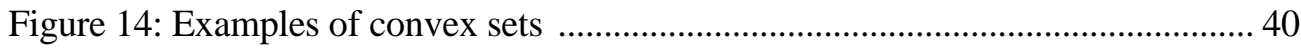

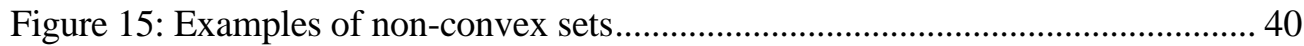

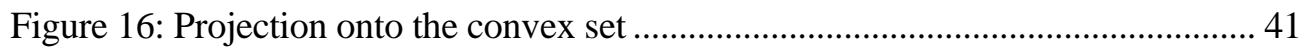

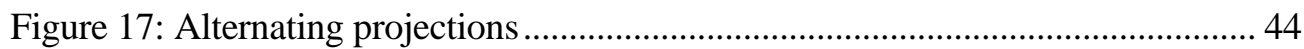

Figure 18: An image degradation model .................................................................... 49

Figure 19: Techniques for blind image deconvolution ................................................ 51

Figure 20: Feedback system diagram for blind image deconvolution.......................... 52

Figure 21: Results of the blind deconvolution algorithm and the MSE ...................... 53

Figure 22: Anisotropic coherence enhancement of fingerprints................................ 57

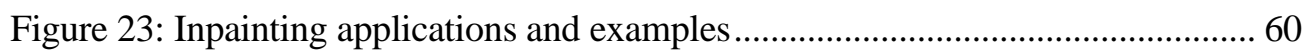

Figure 24: Right choice of inpainting direction ........................................................... 62

Figure 25: Relation between a low resolution and a high resolution image

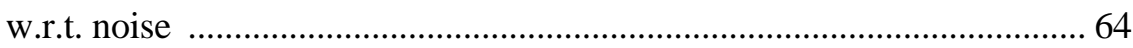

Figure 26: Image interpolated by bilinear interpolation and by a super resolution technique 64 
Figure 27: Proposed super resolution process ...............................................................6 66

Figure 28: Iris image alignment using phase correlation ............................................. 75

Figure 29: Fingerprint image alignment using phase correlation ............................... 76

Figure 30: POCS based image interpolation .............................................................. 77

Figure 31: Blind Deconvolution Restoration (example i)........................................... 78

Figure 32: Blind Deconvolution Restoration (example ii) ......................................... 79

Figure 33: Coherence Enhancement (fingerprint : whorl) …....................................... 79

Figure 34: Coherence Enhancement (fingerprint : loop) ………................................. 80

Figure 35: Image inpainting (filling holes and scars)............................................... 81

Figure 36: Super Resolution process for case (i) Arch ............................................. 82

Figure 37: Super Resolution process for case (i) Whorl .............................................. 83

Figure 38: Super Resolution process for case (i) Iris .................................................. 84

Figure 39: The four copies of the fingerprint which are used to construct

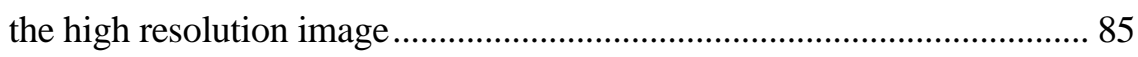

Figure 40: Visual comparison between the original image and the reconstructed image from low resolution copies (case ii)........................... 86

Figure 41: Visual comparison between the original image and the reconstructed image from low resolution copies (case ii).......................... 87

Figure 42: Resolution improvement for detecting pores case (iii) Loop...................... 88

Figure 43: Resolution improvement for detecting pores case (iii) Whorl ................... 89 


\section{LIST OF TABLES}

Table 1: Comparison of theoretical and achieved affine parameters for iris image.....75

Table 2: Comparison of theoretical and achieved affine parameters for fingerprint....76

Table 3: Comparison of mean square errors for fingerprint images..................86 
Introduction

\section{Chapter 1}

\section{Introduction}

\subsection{Problem Statement}

Resolution of biometric images (viz., fingerprint or face) is a problem which has gained importance in recent years. There has been a debate to determine the optimal resolution of fingerprint images, as investing in high quality sensors give a better resolution thus enabling the recognition algorithm to detect singular points (i.e. core and delta) more reliably and accurately. But, with high resolution images, one has to deal with the added computational time and storage space. Thus, choosing the optimal size of the images is a crucial issue. The high quality sensors also imply a higher manufacturing price. Thus, though a better resolution of images has a variety of advantages; one has to also look at the feasibility of investing in them.

To deal with this problem, more recent trend is towards the development of high quality images from multiple low quality images. One of the advantages of doing this is that the developer can still use the low quality sensors available of-the-shelf, obtain multiple copies of the same biometric, and then use them to construct a high quality image. Another application is feature selection/image coding. With the proposed method, it will take a fewer bits to compress a biometric image and thus achieve the necessary coding gain [1].

The process of using multiple aligned frames to create one high resolution image is called super-resolution. Existing super-resolution techniques incorporate specific knowledge about the camera's optics and CCD array density. They determine how the low resolution image was created, by estimating the distortion operations, such as blurring and warping, 
performed by the camera. Most of these algorithms require the accurate alignment of multiple frames. Frame alignment is achieved by image registration. We have used the Fourier analysis for the frame alignment.

The high resolution image thus constructed may be blurred to a certain degree. This image then undergoes a sequence of enhancement techniques viz anisotropic coherence enhancement, blind deconvolution, and inpainting to give the desired high quality image.

\subsection{Scope}

In the image sequence corresponding to multiple copies of the same fingerprint, it is easy to see that the obtained images will have translational and rotational differences with respect to each other. The process of aligning these multiple images onto a high resolution grid is called image registration. For the time being, we consider the transformations to be rigid in nature and not elastic as may be the situation in case of fingerprints. We have assumed that there is no scale difference between the images. Thus, the affine parameters relating two copies of the same fingerprint are the translational and the rotational offsets.

After the alignment of the multiple copies onto the high resolution grid, the next step is to fill out the holes that the process has left behind. This is done by a novel method of image interpolation where each copy of the fingerprint is considered to be one coset of the image subspace. Here we assume that the cosets have a uniform shift with respect to each other and non-linear distortions involved between the copies are absent.

This high resolution image needs to be tuned to obtain a useful image. For this, we use three different enhancement processes. The first technique, blind deconvolution, restores linearly degraded images without explicit knowledge of either the original image or point spread function. This is then coupled with an edge enhancing diffusion, which is based on the idea that a better estimate of the perceptual significant orientation of the edge 
direction can be obtained by constructing a diffusion tensor based on an orientation estimate derived from observing the image at a larger scale. The name "edge enhancing diffusion' was introduced by Weickert [2], although it might be better called 'edge preserving smoothing'. Finally an inpainting algorithm fills in any holes or blotches that may have been introduced due to the super resolution process.

\subsection{Background and Related Work}

\subsubsection{Biometrics}

As the society is becoming more concerned about the personal as well as public security due to increase in frauds, the need for robust, reliable and inexpensive personal authentication is mounting [2a]. Reliable authentication systems guarantee that only authorized users can access the application or service [2b]. Such authentication schemes grant secure access to vulnerable places such as research institutions and financial organizations, to confidential data/information, and applications like ATMs, workstations, and cellular phones [2c]. Authentication systems also find great importance in the forensic applications like criminal identification and prison security.

Passwords (knowledge-based security) have been traditionally used to verify authenticity of the user. However, passwords are secure only as long as they are not guessed or disclosed to a possible imposter. The simpler the password, the easier it is for the legitimate user to memorize and the worse it is for the security, as it can be easily guessed by an attacker [2c]. Personal ID cards (token-based security) is another way of personal identification. However, with token-based authentication systems, security is often questionable when ID cards or tokens are stolen or lost. A robust security system certainly needs the information about 'who you are' than just 'what you know' or 'what you possess'. Biometrics is a rapidly evolving technology which provides the basis for the robust authentication. 
Biometrics refers to an automated method of identification (or verification) of an individual on the basis of certain physiological or behavioral characteristics [2a, $2 b, 2 c$, 3]. As Figure 1 exemplifies, authentication systems make use of characteristics such as fingerprint, face, hand geometry, iris, retina, signature, voice signature, and gait for identifying the legitimate users.

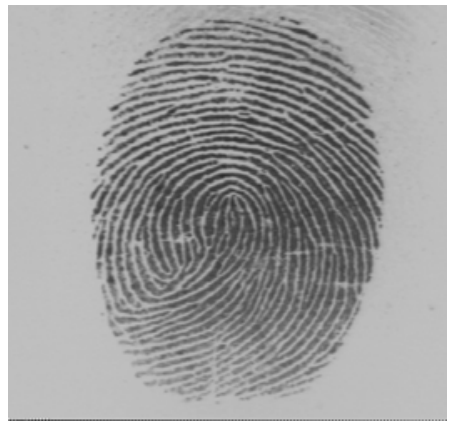

(1) Fingerprint

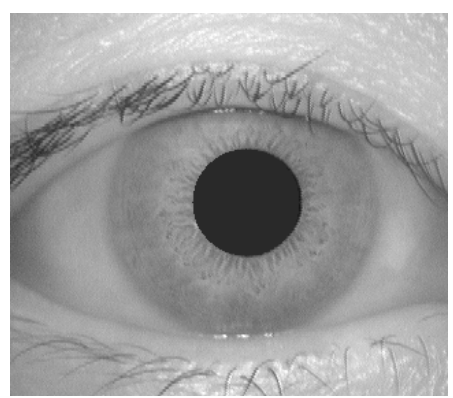

(4) Iris

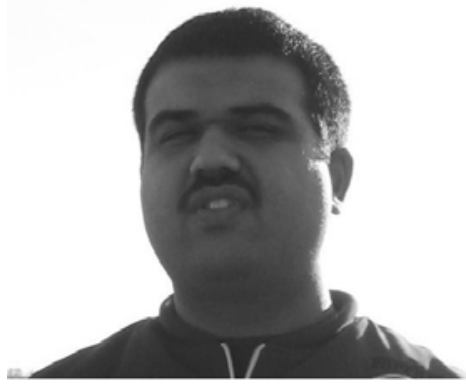

(2) Face

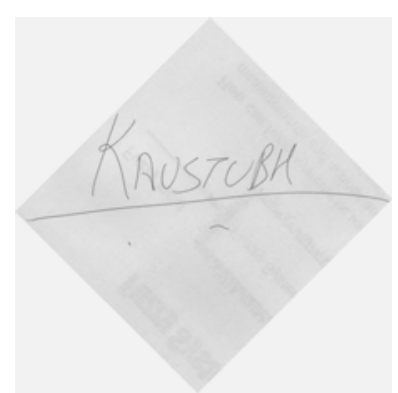

(5) Signature

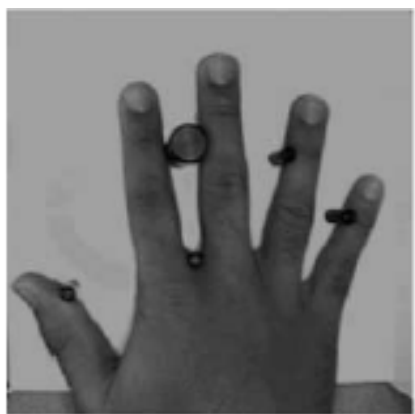

(3) Hand Geometry

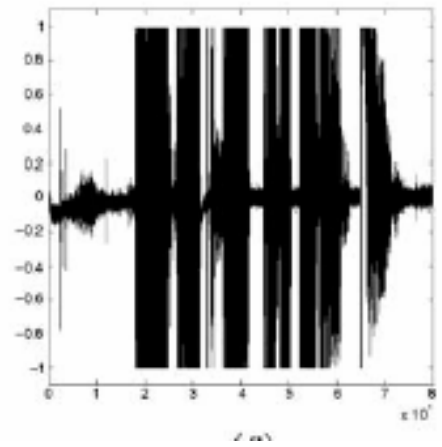

(6) Voice

Figure 1: Examples of some biometric traits associated with an individual

The primary goal of biometrics in authentication systems is to provide identity assurance or the capability to accurately recognize individuals, with greater reliability, speed and convenience at lower costs. Biometrics have an advantage over the traditional authentication methods in a sense that they are difficult to steal, replicate, or share [2c]. Robustness and distinctiveness are the key features that motivate the rapid development of biometrics.

A typical biometric system first acquires biometric data, extracts feature set from the acquired data, and then compares it against the template feature sets in the database. A biometric system operates in two modes: 
Introduction

(i) Authentication (verification), and

(ii) Identification (recognition) [2c].

In the authentication or verification mode, the biometric system compares the acquired biometric signature or the feature set against the template feature set corresponding to the claimed identity, residing in the database, to determine whether the claimed identity is correct or not. In an identification mode or recognition mode, the acquired feature set is compared against the templates corresponding to all the entries in the databases (enrolled users).

In general, a simple biometric system comprises of the following components (see Figure 2) [3]:

(1) Sensor module: It represents the acquisition unit of a biometric system. It captures biometric data of an individual either by enrolling in the system or by requesting an access or service.

(2) Feature extraction module: It extracts a feature set from the acquired biometric data in order to generate a biometric signature for the user.

(3) Matching module: It compares the acquired feature set with the template feature set in the database and generates matching scores.

(4) Decision-making module: The matching scores aid in determining the identity of a user or verifying a claimed identity.

The False Acceptance Rate (FAR) and the False Rejection Rate (FRR) are used as the performance indicators of accuracy and reliability in a biometric system. The FAR describes the fraction of times the unauthorized users are accepted, whereas, the FRR defines the fraction of times the authorized user are rejected by the authentication system. The lower the FAR and FRR, the better is the rating of the biometric system [4].

The effectiveness and suitability of a particular biometric for a specific access control application vary significantly. Among all the factors determining the suitability of a biometric for an application, the user acceptability is the utmost important factor. The 
user acceptability and the distinctiveness of a biometric are interrelated. A biometric with high distinctiveness may not be acceptable by the users because of its ability to violate an individual's privacy. In such situations, it is suggested to use a biometric with less distinctiveness, just enough to verify the person's identity [5].

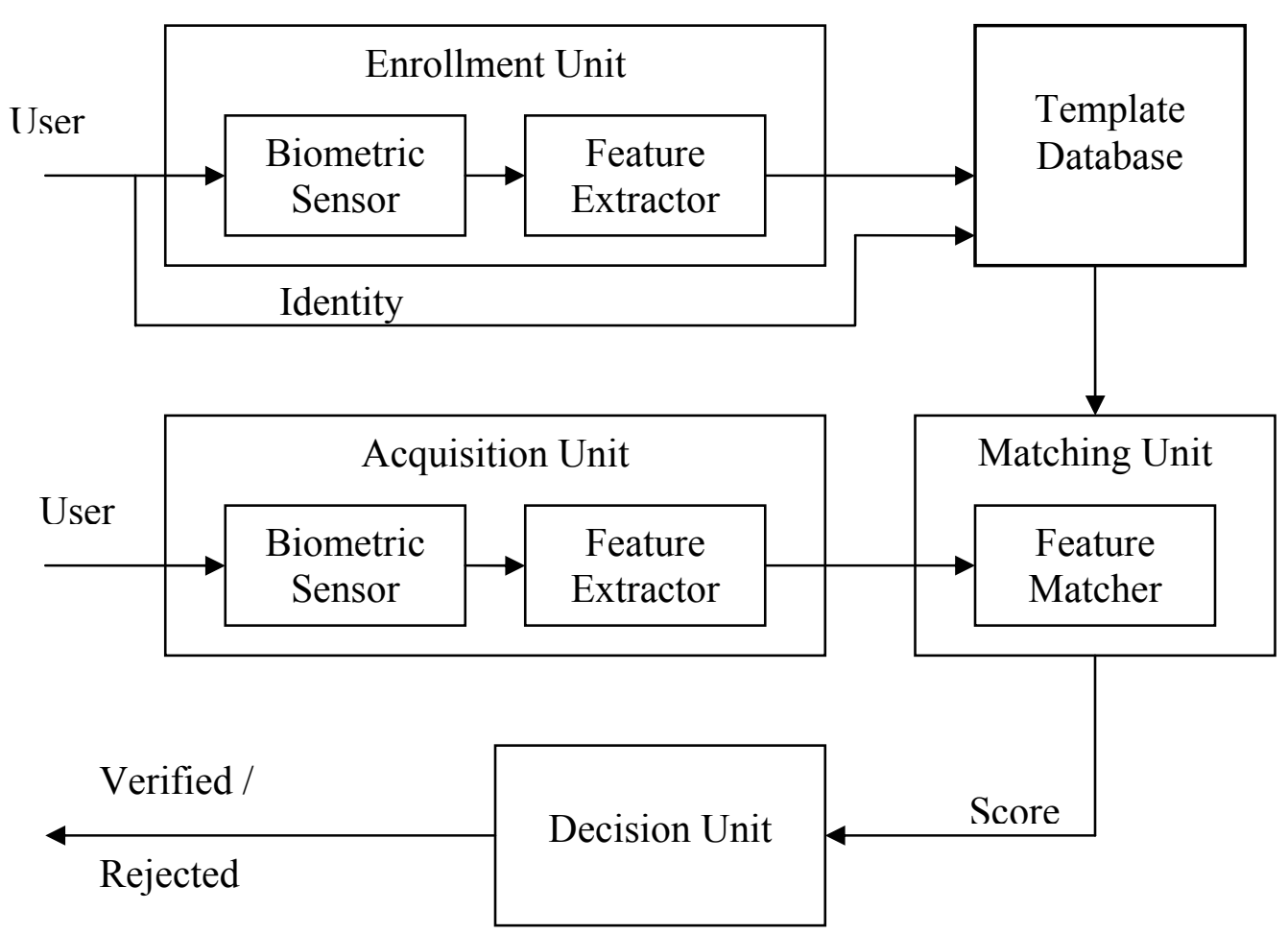

Figure 2: Components of a simple biometric system

The following subsections introduce some of the popular and effective biometrics.

\subsubsection{Fingerprint}

Among all known biometrics, fingerprint is one of the oldest and widely recognized biometric trait. Reference to the use of fingerprints for the identity purpose, as a concept, dates back over about thousand years. In East Asia, potters put their fingerprint marks on their wares as brand identity [3]. Fingerprint represents the flowing pattern of ridges and furrows (also known as valleys) located on the surface of a fingertip (Refer Figure 3). 
In earlier days, the inked impressions of a fingertip on paper have been used to acquire fingerprint images. A wide range of fingerprint sensors is available these days. These sensors can be integrated in a mouse or keyboard also [6]. The use of fingerprints for automatic user-authentication systems for civilian and commercial applications has increased as a result of the development of compact solid-state fingerprint sensors.

Fingerprint for each person is believed to be unique. The uniqueness of a fingerprint is exclusively determined by the ridge pattern and the local ridge characteristics. The flow of ridge pattern in the central region of the fingerprint defines the global characteristics. The core and delta points are important global features. The core point is defined as the highest point on the innermost ridge, while, the delta point is defined as the point where three flows meet.

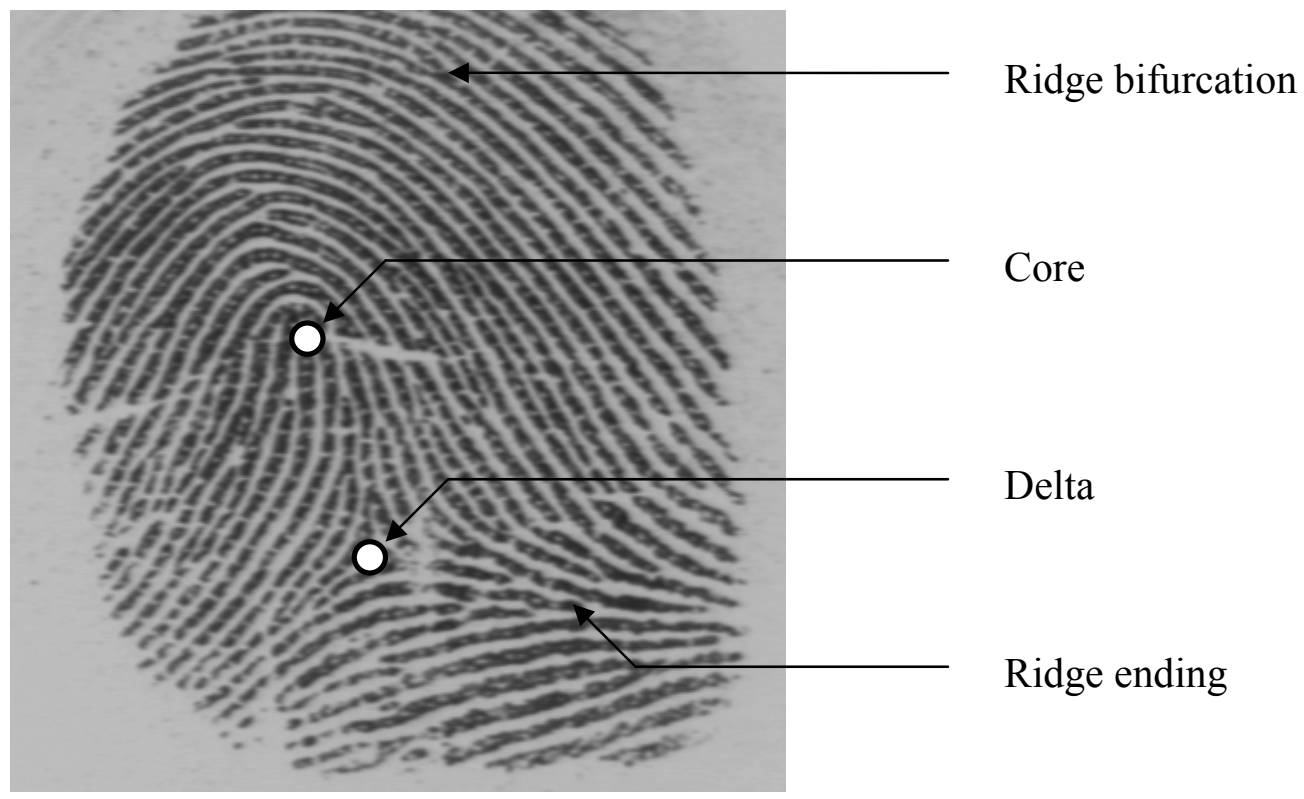

Figure 3: Features of a fingerprint

The abnormalities in the ridge structure, called minutiae, define the local ridge characteristics. Ridge ending and ridge bifurcation are the two most prominent local ridge characteristics. A good quality fingerprint typically contains nearly 40-100 minutiae. The location and the distribution of minutiae points are used to match the fingerprints. When an automatic fingerprint identification system is used in identification mode, the global ridge characteristics of the query fingerprint image are used to limit the search to one 
specific class of the database and the local characteristics like minutiae are used to find out the exact match [4].

Fingerprint matching techniques fall under two main categories: minutiae-based and correlation-based [4]. Minutiae-based matching techniques extract minutiae points and use their relative positions for fingerprint matching. The performance of these techniques is highly dependent on the accuracy of minutiae extraction. These techniques are not suitable for matching poor quality fingerprints. The correlation-based techniques consider the global ridge pattern of the fingerprint to match fingerprints.

It is a well accepted fact that fingerprint recognition systems have a good ability to accurately confirm user's identity, and thus, currently fingerprint is the most widely used biometric.

\subsubsection{Hand Geometry}

As the name suggests, hand geometry uses the geometric structure of the hand for the authentication. The geometric structure of the hand is defined by the features such as width of the fingers at different locations, width of the palm, thickness of the palm, surface area of the palm, length of the fingers, etc [5] (refer figure 4). Authentication of an identity of an individual is based on these higher level features of the hand.

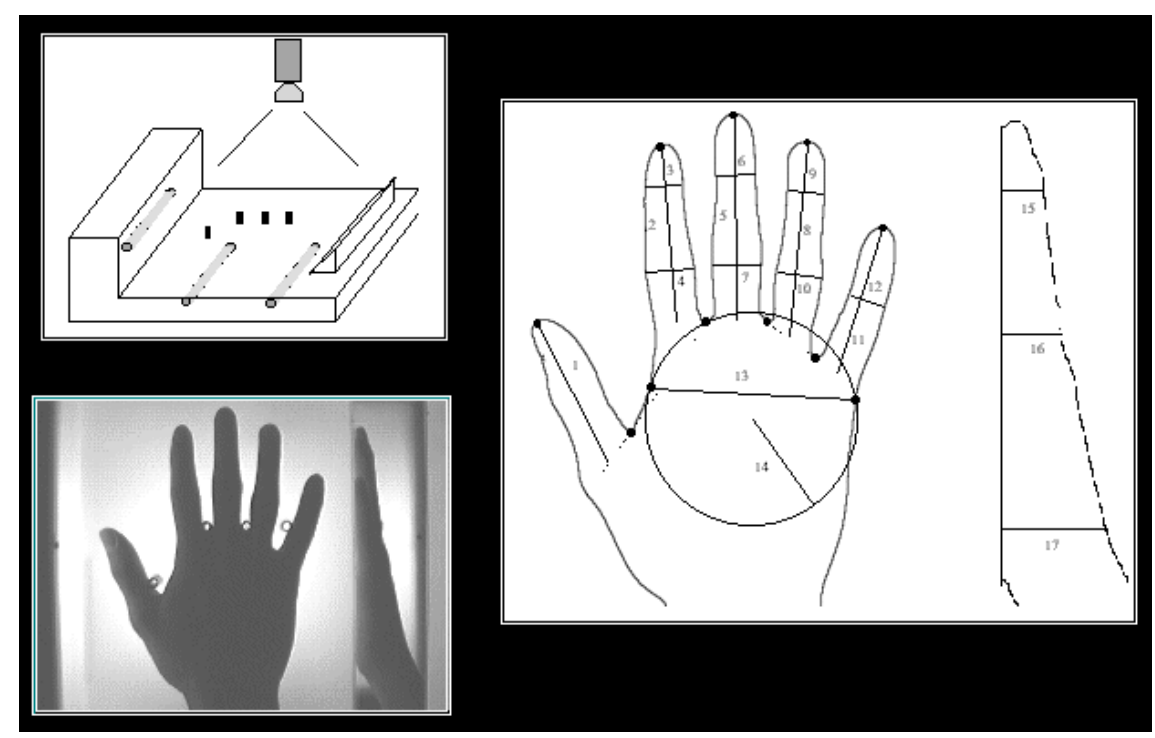

Figure 4: Hand Geometry features and acquired image [6a] 
Since hand geometry does not vary significantly across the population, it is typically used only for the verification of a person's identity. This results in higher user acceptability for hand geometry as a biometric [5]. Hand geometry data is easier to collect compared to other biometrics. One concern with hand geometry in authentication is its susceptibility to hand injuries.

\subsubsection{Face}

Facial biometrics is another promising user authentication technology that is commercially used. It uses various features of the face to recognize or verify an authorized user. The primary step in the facial recognition or identification is a face detection process in which the location and spatial content of the face is determined within the given image. Face detection is a difficult process as it involves high degree of variability associated with human faces because of color, texture, expressions, poses, accessories, etc., and also because of complex background [2c]. Face detection also needs to deal with the changes in the lighting conditions.

Face recognition algorithms generally uses the eigenface approach to extract features from the face (Figure 5). It computes the feature vector of an original query face by finding its projection on the lower dimensional eigenspace [2c]. In the matching stage, the system compares the eigenface coefficients of the detected face with the template to find the best match. Local feature analysis is another class of facial recognition algorithms in which a set of geometric metrics and distances from the facial image are extracted [3].

Though face is a biometric which is the most common choice, the problem with two dimensional images is susceptibility to spoofing and variance with the change in posture, lightening and distance of face from the camera. To counter this, three dimensional face matching is an emerging field. 
Introduction

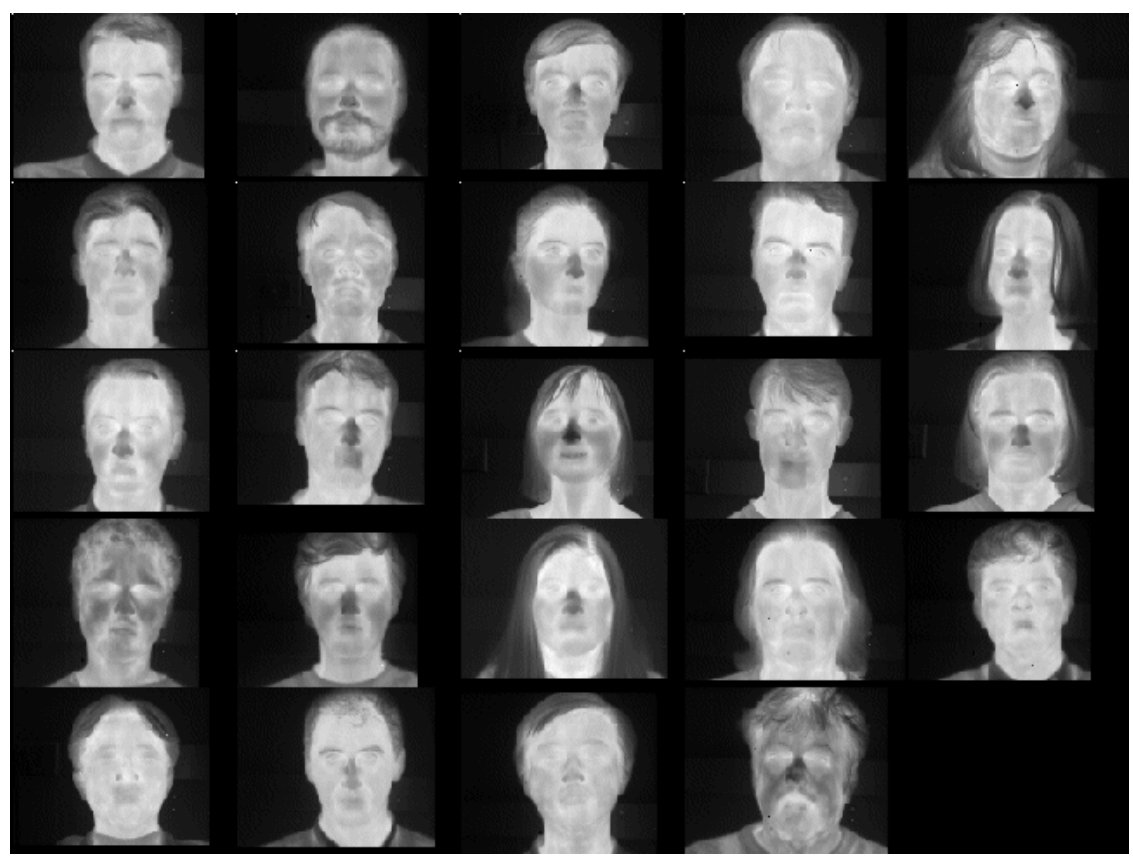

Figure 5: Principal component analysis for face recognition, the eigen vector approach [6b]

\subsubsection{Iris}

The iris is the ring of colored tissue surrounding the pupil. The highly intricate and randomized nature of the iris makes it exceptionally suitable as a biometric [7]. Iris is also genetically independent making it highly distinctive. An iris image is converted into a numeric code known as iriscode, which can be easily manipulated. It also permits efficient comparisons of irises.

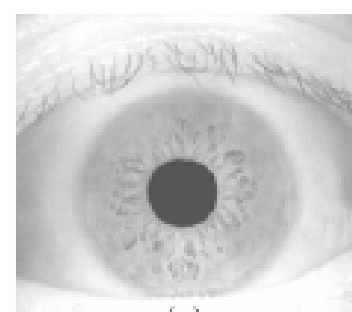

(a)

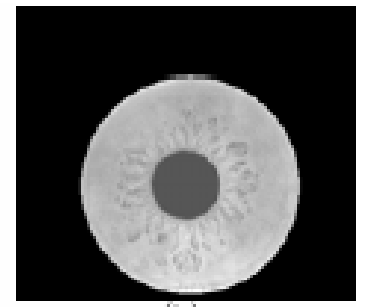

(b)

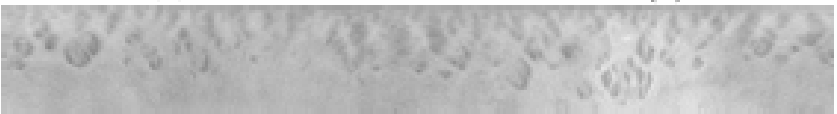

(c)

Figure 6: (a) original iris image, (b) localized iris image, (c) processed iris image [25] 
Once the properly focused and well positioned image of the iris is obtained, an iriscode is generated using a set of Gabor filters with eight circular bands as can be seen in figure 6 . At the verification or identification stage, the iriscode derived from the iris image is compared with the iriscodes from the database on the basis of Hamming distance [7].

In general, iris recognition systems are much more expensive than other biometric systems because of the requirement of high resolution scanning and infrared illumination. However, whenever very high degree of security is desired, the authentication confidence associated with the iris recognition system makes up for the cost issues.

Even though this work can be used for other biometric traits such as face and iris, we have concentrated on fingerprints as it is the most commonly used biometric for recognition.

\subsubsection{Fingerprint Enhancement}

Fingerprints are graphical ridge patterns present on human fingers, which, due to their uniqueness and permanence, are among the most reliable human characteristics that can be used for people identification [8, 9]. A common hypothesis, confirmed by the experience accumulated during a century of forensic use of fingerprints, is that certain local structures derived from the fingerprint ridges, called minutiae, are able to capture the invariant and discriminatory information present in the fingerprint image [10].

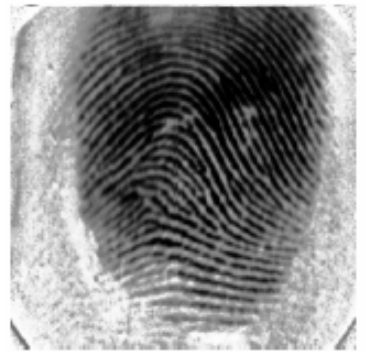

(a)

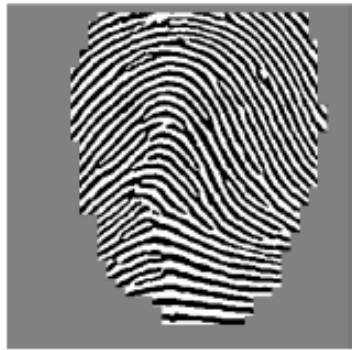

(b)

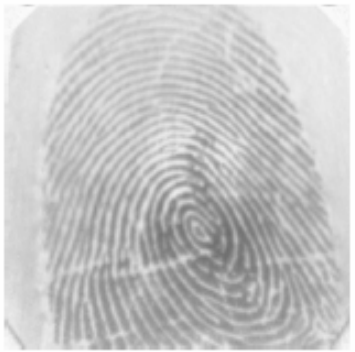

(c)

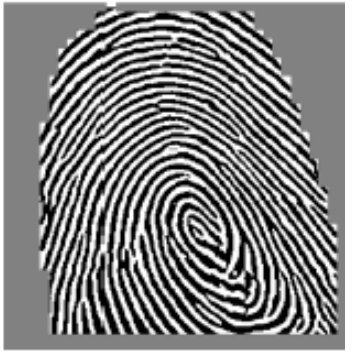

(d)

Figure 7: Images (a) and (c) are enhanced to achieve a better quality image in (b) and (d).

Several factors like the presence of scars, variations of the pressure between the finger and acquisition sensor, worn artifacts, the environmental conditions during the acquisition process, and so forth, can dramatically affect the quality of the acquired 
fingerprint image (see Figure 7). Since minutiae are fine features derived from the ridge pattern, their extraction can become notoriously difficult if the noise generated by the factors described above is not substantially reduced.

The main goals of a fingerprint image enhancement algorithm are

(i) To reduce the noise present in the image, and

(ii) To enhance the quality of fingerprint ridges.

An enhancement algorithm transforms a gray-scale fingerprint image into a binary representation of the ridge pattern called binary ridge-map image. The image pixels located on fingerprint ridges normally exhibit lower gray level intensities than the image pixels located on the fingerprint valleys. Thus, enhancing an image means to classify image pixels as ridge or valley pixels by comparing their intensities with a certain threshold intensity value.

It can be easily seen that such a simple point operation approach will not reduce the noise present in the image because it does not take into consideration the strong correlation that exists between neighborhood intensity values in the fingerprint image pattern. Generally, it is quite difficult to model the noise present in the fingerprint image, but one advantage of fingerprints is that the signal is fairly simple to characterize due to the flow of fingerprint ridges whose orientations are slowly changing in the fingerprint pattern.

Taking a small local area in the fingerprint image we can see that the ridge orientation as well as the ridge period is maintained almost constant inside this area, whereas the noise present in the image does not exhibit such regularities. The majority of the fingerprint enhancement methods proposed in the literature is based on this observation. The basic idea being to design adaptive filters attuned to the corresponding orientation and ridge period in each image region.

In the method proposed by Ratha et al. in [11] the image is smoothed using a onedimensional averaging mask aligned along the local ridge orientation, and the fingerprint 
Introduction

ridges are detected as local minima of the gray-level projection waveform along a scan line perpendicular to the local ridge orientation.

Jain et al. [12] proposed to accentuate local minima intensity values along a direction normal to the local ridge orientation by convolving the fingerprint image with two masks aligned to the ridge orientation. Subsequently, a binary ridge-map image is obtained by comparing the intensity values of the pixels in the two convolved images with a certain threshold value. The enhancement algorithm proposed in [13] by Hong et al., relies on Gabor filters of size $11 \times 11$ pixels tuned to the ridge orientation and ridge period priory estimated in nonoverlapping image blocks of $16 \times 16$ pixels.

Sherlock et al. [14] proposed a directional Fourier domain filtering for fingerprint enhancement. They design a set of 16 directional filters tuned on different orientations between 0 and $\pi$. By applying each filter onto the entire fingerprint image they obtain 16 filtered images. Next, the value of each pixel in the enhanced image is selected from one of the 16 filtered images based on the ridge orientation in the neighborhood of that pixel. Finally, the binary ridge-map image is obtained by a locally adaptive thresholding technique.

Instead of designing filters tuned on corresponding spatial frequency of each image region, Willis and Myers proposed in [15] to use as a filter directly the magnitude of the Fourier transform of the local image region. This magnitude exhibits most of the qualities required from a properly designed enhancement filter since it has a dominant component at the corresponding ridge orientation and frequency, and on the other hand, due to the noise irregularity it exhibits small magnitudes along other components.

\subsubsection{Super Resolution}

Although the topics that we intend to cover have been studied for a long time, hardly any have been applied to biometrics. In computer vision, super resolution is generally thought as inferring the missing high resolution image from the low-resolution image [23, 24]. The interest has been driven by a variety of applications such as video surveillance, 
remote sensing and medical imaging. In general, all these methods are classified as reconstruction based methods [16, 17, 18, and 19] and learning based methods $[20,21$, and 22].

Tsai and Huang [16] first proposed a super resolution method which processed images in frequency domain. Sauer and Allebach [17] modeled super resolution as an interpolation problem with non-uniformly sampled data. Irani and Peleg [18] proposed an iterated back-projection (IBP) method to obtain the high-resolution image by simulating the imaging process via warping so that the difference between the observed and simulated low-resolutions was minimized. Stevenson [19] introduced a maximum-a-posteriori (MAP) method for resolution enhancement. All these methods only make use of weak priors for resolution enhancement [25].

Recently, Freeman [20] incorporated the relationship between middle frequency and high frequency of example images as prior information, and then the Belief Propagation algorithm was used to infer a high resolution image from a given low resolution image. He further introduced a one-pass algorithm for the same problem [21]. Hertzmann [22] proposed an analogy method. Using only a pair of images as training data, he transformed an input image into the resulting image, which has the same relation as the training pair. These learning-based methods obtained significantly better results than existing reconstruction-based algorithms.

However, when these learning-based methods fully learn the intensity prior between all bands of images, some fake high information will be inevitably introduced into the synthesized high resolution image though this information can give us a good visual effect. For super resolution, three works come to the forefront immediately. They are the outstanding papers by Capel and Zisserman [26], Irani and Peleg [18] and the one by Baker and Kanade [23]. The following section covers the introduction of their work and its significance in our approach. 
The objective of Capel and Zisserman's work is the super-resolution enhancement of image sequences. In particular, they consider images of scenes for which the point-topoint image transformation is a plane projective transformation. An imaging model and a maximum likelihood (ML) estimator of the super-resolution image are described. Then two estimators suitable for the enhancement of text images: a maximum a posterior (MAP) estimator based on a Huber prior, and an estimator regularized using the Total Variation norm are proposed. The effects of a poorly estimated point spread function (PSF) on the super resolution result are also tackled and conditions necessary for this parameter to be included in the optimization are explained.

Kanade and Baker on the other hand, proposed a super-resolution algorithm that uses a different kind of constraint, in addition to the reconstruction constraints. Their algorithm attempts to recognize local features in low resolution images and then enhances their resolution in an appropriate manner. They describe their super-resolution algorithm as a hallucination or reconstruction algorithm. They state that all super-resolution algorithms are based on the fundamental constraints that the super-resolution image should generate the low resolution input images when appropriately warped and down-sampled to model the image formation process. They also derive a sequence of analytical results which show that the reconstruction constraints provide less and less useful information as the magnification factor increases.

\subsubsection{Image Alignment}

Previous subsection briefly described a number of prominent works in the field of super resolution. Before delving into it, let us consider the techniques of image registration or alignment which have been used as an initial step for super resolution. According to Brown [26a] who gives an accurate survey of the image registration techniques, two major types of distortions are distinguished which facilitate the need for alignment.

The first type of misregistration is the source, which is the cause of the misalignment between the two images. These types of distortions determine the transformation class which will optimally align the two images. This class will in general influence the 
technique to be employed. The second class is those in which the source is not the cause of misalignment. The distortions of the second type normally affect intensity values but may also results in spatial distortions. These cannot be removed by registration but may make it more difficult. They affect the choice of feature space, similarity measure, and search space strategy which makes up the technique to solve them. We are going to concentrate on the first class of problems and thus the second class will not been considered at all.

For the registration, it is imperative that the following points be considered,

- Sensor noise;

- Perspective changes from sensor viewpoint or platform perturbations;

- Object changes such as movements, deformation and growth;

- Different sensors.

This broad range of methodologies makes it difficult to classify and compare techniques since each technique is often designed for specific applications and not necessarily for specific types of data or problems. But most of these techniques involve searching over the space of transformations of a certain type to find the optimal transformations for a particular problem. The traditional technique of the cross correlation function and similar methods like the statistical correlation, matched filters, the correlation coefficient, and sequential techniques are typically used for small well defined affine transformations. They are often productive for a single translation.

The second class of techniques used for the affine transformations, in cases where frequency dependent noise is present, is the Fourier methods. The Fourier Transform has several properties that can be exploited for image registration. Translation, rotation, reflection, distributivity property, and scaling, all have their counterparts in the Fourier domain. In addition, the frequency domain gives an excellent robustness against correlated and frequency dependent noise. 
For a global transformation method which may be non affine, the primary approach is the feature point mapping to define a polynomial transformation. The point mapping technique is the primary approach adopted currently to register two images whose type of misalignment is unknown. This is also better known as the minutiae matching in the biometrics fingerprint domain. This method generally consists of three stages. Initially features in the image are computed. In the next stage, feature points in the reference image are corresponded with feature points in the data image. In the final stage, a spatial mapping is determined using these matched feature points based on the least squares regression technique.

In a complex case where the registration technique must determine a local transformation when legitimate local distortions are present, techniques based on specific transformation models such as an elastic membrane are used. This is the most recent trend in image registration. Instead of applying piecewise interpolation to compute a transformation to map the control points of one image onto another, these methods model the distortion in the image as the deformation of an elastic material. Still, the methods of piecewise interpolation are closely related since the energy minimization needed to satisfy the constraints of the elastic model can be solved using splines.

Summarizing the different image registration methods introduced above, we see that the methods are characterized by the complexity of their corresponding transform class. This transformation class can be determined by the source of misalignment. The methods are then limited by their applicability to this transformation class and the type of distortions they can tolerate.

\subsubsection{Image Interpolation}

The next logical step in the construction of super resolution images after the image registration is to map these registered images on the high resolution grid and interpolate them as the need may be. In the area of image interpolation by far the most well known and widely used techniques are those of polynomial or Lagrange interpolation and 
interpolation based on splines. These image models are based on the assumption that locally each image behaves like an $n^{\text {th }}$ degree polynomial [27]. Whether separable or nonseparable, these methods can be efficiently implemented using an up-sampler followed by a filter. Examples of such interpolation algorithms are cubic [28] and other spline based methods [29]. These polynomial image models have the advantage of being fast but they tend to introduce serious jaggedness (the staircase effect) and blur. They are the choice of image manipulation programs such as Adobe Photoshop and GIMP.

There have been many attempts at improving the local polynomial image models in order to enhance edges and the overall image sharpness. In [30] the author introduces the concept of warped distances to adaptively adjust the bi-cubic interpolation filter. By assuming a different relative location (a warped distance) of the four known samples with respect to the interpolated sample, the filter coefficients can be changed in order to sharpen edges. Though the paper claims the removal of artifacts, it is not clear how it addresses the stair casing effect.

Other attempts at modifying the polynomial image model in order to reduce jaggedness and sharpen edges are those of [31] and [32]. Around edges the authors of [31] map a 3by-3 neighborhood about each pixel in the low-resolution image to a best-fit continuous space step edge and then re-sample it at the higher density. Images interpolated with this method look sharper than bi-cubic interpolation but often look too much like drawings, especially for zoom factors of four or more. In [32] the authors use an iterative rendering and correction step for edge directed interpolation and claim that this produces sharper images.

A second class of image interpolation algorithms includes those based on multiresolution analysis. The authors of [33] model the wavelet coefficients of a dyadic (nondecimated) wavelet transform using exponential decay. In particular, they show that the local maximum of the dyadic wavelet coefficients decreases exponentially from coarse to fine scales. The authors of [34], [35] apply the wavelet exponential decay model to image interpolation by posing the interpolation problem as one of estimating the fine detail 
wavelet coefficients. Exponential decay is estimated from the coarse scale coefficients. If an exponential decay is detected, an estimate of the fine scale wavelet coefficient is made based on the estimated decay, otherwise the estimate is zero.

The super-resolution image models of [36], [37] can also be described as interpolation methods based on adding image details in the wavelet domain, although the details are added based on training data and not wavelet decay. Using high resolution training data, images are decimated and table lookups of low-resolution/high-resolution patches are built. These methods produce sharper images and can work well, given decent training data, but their main drawback is the potential for introducing artifacts when the table lookup procedure chooses a wrong high resolution patch.

In addition, the image models may not work as well for data containing artifacts, such as JPEG compressed images, as the methods tend to consider artifacts as part of the image and may enhance the artifacts more than the image itself. The image models of [38] are similar in nature. The authors of [39] model the wavelet coefficients using Gaussian mixtures and apply their models to image denoising [40]. In [41] the image model of [39] is extended to image interpolation. For this method, the interpolation results are comparable with a bi-cubic interpolation. One particular feature of this approach is that the detail wavelet coefficients are realizations of estimated Gaussian mixtures. Hence, every run of the algorithm produces a different result. The weakness of this model, in image interpolation, lies primarily in the determination of the Gaussian mixture parameters.

The authors of [42] propose a maximum a posteriori (MAP) pixel estimation technique which results in the optimization of convex functionals. Their nonlinear image expansion technique sharpens edges but the interpolation technique does not eliminate jagged edges. The work of [43] also uses a MAP framework for estimating a high resolution image from a sequence of under-sampled images. Their expansion method could be used for a single image by assuming only one frame. 
In [44] the authors present a least squares edge directed interpolation method. The method assumes that each pixel is a linear combination of its neighboring pixels. Further, it is assumed that locally the weights are constant. A linear system of equations is solved in order to find the local weights. This method works for interpolation by factors of two and performs well around edges, but performs poorly in high frequency regions, sometimes introducing undesired artifacts. In [45] it is shown how this solution can be reformulated using optimal recovery which allows for additional assumptions about the local derivatives in addition to the known local pixels to be used in the interpolation process.

In [46], the authors pose the image interpolation problem as one where the image belongs to a fixed quadratic image class. To solve the interpolation problem the authors add some known linear partial differential constraints. The paper develops adaptive quadratic signal classes to better model the local image behavior. These were the recent developments in the image interpolation domain. The method which we have developed for this purpose is a variation of these methods and has not been proposed before. The method will be explained in detail in the next section.

\subsubsection{Image Restoration}

After these two steps of Image Registration and Image Interpolation the third step is Image Restoration which tries to remove the artifacts generated in the high resolution image during the first two steps. Thus in short, in the registration stage, the relative shifts between the low resolution images compared to the reference low resolution image are estimated with fractional pixel accuracy. Obviously, accurate sub pixel motion estimation is a very important factor in the success of the high resolution image reconstruction algorithm. Since the shifts between low resolution images are arbitrary, the registered high resolution image will not always match up to a uniformly spaced high resolution grid. Thus, nonuniform interpolation is necessary to obtain a uniformly spaced high resolution image from nonuniformly spaced composite of low resolution images. Finally, image restoration is applied to the up sampled image to remove blurring and noise. 
Image restoration, as can be seen in Figure 8, may be viewed as an estimation process in which operations are performed on an observed or measured image field to estimate the ideal image field that would be observed if no image degradation were present in the imaging system. In order to effectively design a digital image restoration system, it is necessary to characterize quantitatively the image degradation effects of the physical imaging system, the image digitizer, and the image display [47].
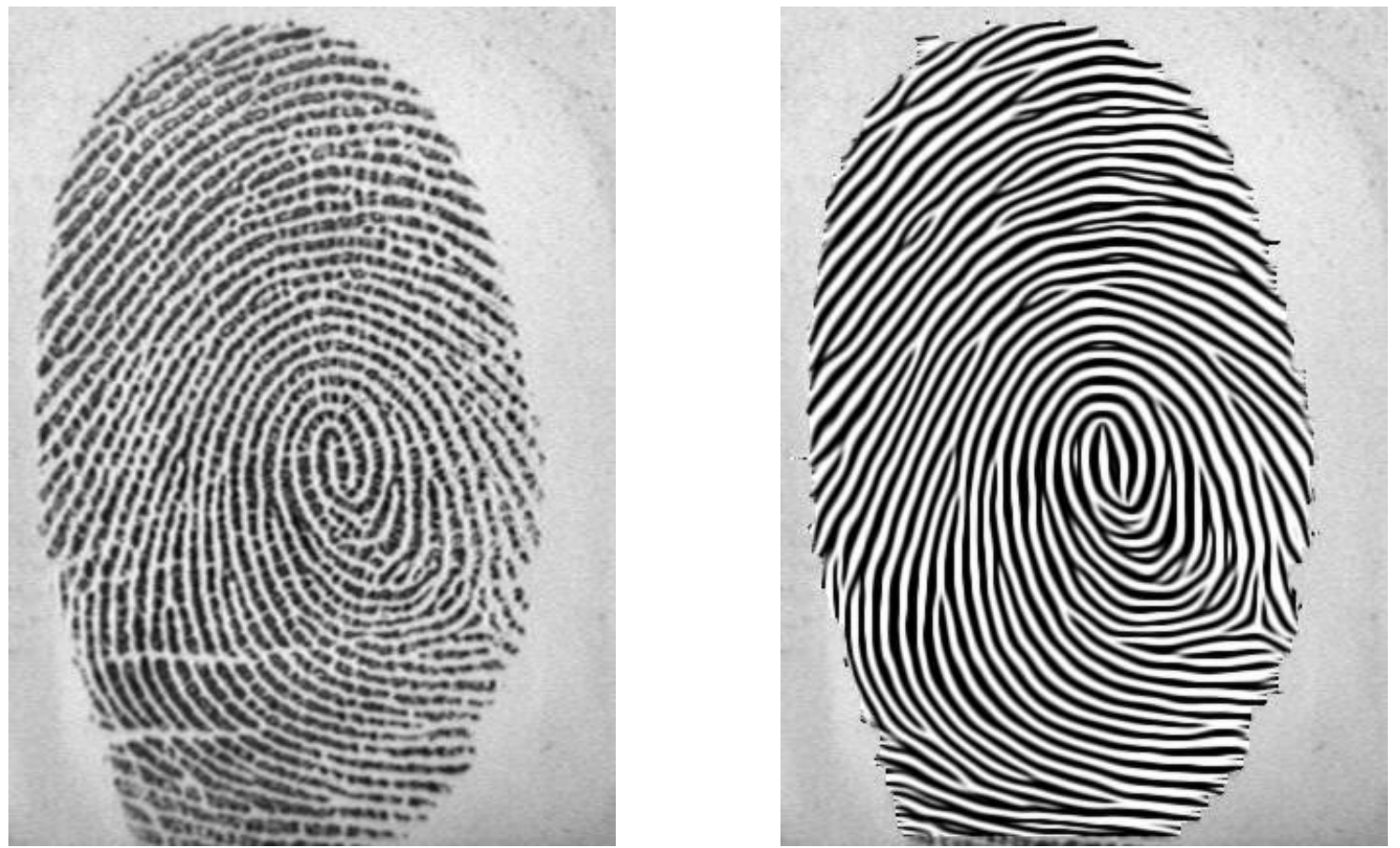

Figure 8: Image restoration results

The use of partial differential equations and curvature driven flows in image analysis has become an interest raising research topic in the past few years. The basic idea is to deform a given curve, surface or image with a partial differential equation (PDE), and obtain the desired result as the solution of this PDE. Sometimes, as in the case of colored images, a system of coupled PDE's is used. The art behind the technique is the design and the analysis of these PDE's [48]. 
PDEs can be obtained from variational problems. Assuming a variational approach to an image processing problem formulated as,

$$
\arg \left\{\operatorname{Min}_{l} U(u)\right\}
$$

where $U$ is a given energy computed over the image I. Let $F(\Phi)$ denotes the Euler derivative (first variation) of U. Because under general assumptions a necessary condition for $\mathrm{I}$ to minimize $\mathrm{U}$ is that $\mathrm{F}(\mathrm{I})=0$, the local minima may be computed by means of the steady state solution of the equation,

$$
\frac{\partial I}{\partial t}=F(I),
$$

where $t$ is an artificial time-marching parameter. PDE's obtained in this way have already been used for quite some time in computer vision and image processing, and the literature is humongous. A classical example is the Dirichlet integral,

$$
U(I)=\int|\nabla I|^{2}(x) d x,
$$

which is associated with the linear heat equation.

$$
\frac{\partial I}{\partial t}(t, x)=\Delta I(x)
$$

More recently, extensive research is being done on the direct derivation of evolution equations that are not necessarily obtained from the energy approaches. Using partial differential equations and curve surface flows in image analysis leads to modeling images in a continuous domain. This simplifies the formalism, which becomes grid independent and isotropic. The understanding of discrete local nonlinear filters is facilitated when one lets the grid mesh tend to zero and due to an asymptotic expansion, one rewrites the discrete filter as a partial differential operator.

Conversely, when the image is represented as a continuous signal, PDEs can be seen as the iteration of local filters with an infinitesimal neighborhood. This interpretation of PDEs allows one to unify and classify a number of known iterated filters as well as to derive new ones. Actually, we can classify all the PDEs that satisfy several stability requirements for image processing such as locality and causality. 
Another important advantage of the PDE approach is the possibility of achieving high speed, accuracy and stability with the help of the extensive available research on numerical analysis. When considering PDEs for image processing and numerical implementations, we are dealing with derivatives of non smooth signals, and the right framework must be defined. The theory of viscosity solutions provides a framework for rigorously using a partial differential formalism, in spite of the fact that the image may not be smooth enough to give a classical sense to derivatives involved in the PDE. This area has a unique level of formal analysis, giving the possibility of providing not only successful algorithms but also useful theoretical results such as existence and uniqueness of a solution.

Ideas on the use of PDEs for image processing go back at least to Gabor [49] and, a bit more recently to Jain [50]. However, this field came to prominence after the works of Koenderink [51] and Witkin [52]. These researchers rigorously introduced the notion of scale and space. Their seminal work is to a large extent the basis for most of the research in PDEs for image processing. In their work, the mutiscale image representation is obtained by Gaussian filtering. This is equivalent to deforming the original image by means of the classical heat equation, obtaining in this way an isotropic diffusion flow.

In the late 1980s, Hummel [53] noted that the heat flow is not the only parabolic PDE that can be used to create a scale space, and indeed he argued that an evolution equation that satisfies the maximum principle will define a scale space as well. Maximum principle appears to be a natural mathematical translation of causality. Koendernik made another significant contribution to the field when he suggested adding a thresholding operation to the process of Gaussian filtering. As later suggested by Osher, and his colleagues, this leads to a geometric PDE, one of the most famous ones: curvature motion.

The work of Perona and Malik [54] on anisotropic diffusion has been one of the most significant and influential work in this area. They proposed replacing Gaussian smoothening, equivalent to isotropic diffusion by means of the heat flow, with a selective 
diffusion that preserves edges. In the same frame work, the seminal works of Osher and Rudin on shock filters [55] and Rudin et. al. [56] on total variational decreasing methods explicitly stated the importance and the need for understanding PDEs for image processing.

Many of the PDEs used in image processing and computer vision are based on moving curves and surfaces with curvature based velocities. The basic idea is to represent the deforming curve, surface or image as the level set of a higher dimensional hyper surface. This technique not only provides more accurate numerical implementations but also solves topological issues that were previously difficult to treat. The representation of objects as level sets is not new to the computer vision and image processing communities, as it is one of the fundamental techniques in mathematical morphology [57].

Thus, the frameworks of PDEs and geometrically driven diffusion have been applied to many problems in image processing, and computer vision after the seminal works mentioned above. We have concentrated on PDEs for the application of fingerprint (image) restoration after creating the high resolution image. We have used different PDEs for the two problems of anisotropic coherence enhancement of images and image inpainting.

\subsection{Motivation}

With Biometrics gaining importance in the recent past, a large number of researchers from different fields have started exporting their ideas into the biometric framework to develop a better system than the previous everyday. Biometrics being a broad area, there is scope for everyone. Researchers with a background in various fields such as sensors, image processors, pattern recognition, database management, etc. have contributed in the development of biometric systems. 
A similar motivation has prompted us to develop a super resolution algorithm for the biometric images. Even though we mentioned image processing earlier, it is still in itself a broad process. Various methods of image enhancement can be applied to biometric data. The motivation behind developing a super resolution algorithm for different biometric traits is to enable the use of low quality of-the-shelf sensors which are readily and cheaply available in the market and to construct an image which would aid in the recognition step. These improved resolution images are comparable to the images acquired by the expensive high resolution images.

With the importance gained recently by using pores on the ridge patterns to aid in efficient matching algorithm, the use of high resolution finger prints has been thought of. Thus an efficient and cost effective resolution enhancement algorithm will definitely facilitate in the identification of these pores.

Another set of applications for super resolution techniques includes surveillance applications. The camera inherently is placed high above the ground and thus the subject's face is hardly a few pixels wide in the acquired image. A resolution algorithm will refine the image in general and, thus, a portion containing the face, resulting in data of higher quality for recognition purpose.

This work concentrates on the enhancement of the raw biometric images prior to feeding them into a template generation module. We have adopted, modified, and integrated a large set of ideas from the signal processing domain to ultimately give us a robust super resolution algorithm.

As stated earlier, the Super Resolution process is divided into two main parts, viz. the image registration and the image restoration. For the image registration we have chosen to use a global alignment process. We have used the global phase correlation method for its simplicity of use and faster implementation time, not compromising however on the quality of the results. A Projection onto the convex sets (POCS) interpolation method guarantees the convergence of a solution and thus is the best choice for this application. 
Introduction

For the image restoration, we have chosen the blind deconvolution method, as it does not assume any blurring kernel for the restoration and thus, is the right choice for the applications with no knowledge of blurring kernels. The anisotropic coherence enhancement uses the PDEs to improve the high resolution component of the images while preserving the edges. Image inpainting is the proven tool for removing holes and scratches from images for a long time and thus it fits in snugly into our application of restoration.

\subsection{Thesis Organization}

After the introduction and the related work to the problem of super resolution for biometric traits, the next chapter deals with the theory of super resolution. The whole problem is divided broadly into two parts. In the first part, a high resolution image is constructed from multiple low resolution ones. In the second, this high resolution image undergoes certain enhancement techniques to construct the required image.

The second chapter deals with the image registration part. This is again subdivided into two parts viz the image alignment and the image interpolation parts. The theory behind image alignment and the various methods are given in this part. Then, some mathematical treatment for the phase correlation method is given. The phase correlation is then applied to the image both in the frequency domain and in the log polar domain. The method thus applied is explained in detail along with the algorithm. The final part of the second chapter deals with the ways to construct a high resolution image by placing the multiple aligned images onto a high resolution grid. This process is a feedback iterative system which will go on improving the image quality each time the loop is run.

The third chapter consists of the enhancement modules which enhances the high resolution image constructed in the previous section and outputs the final image. We have considered mainly three enhancement techniques viz, Anisotropic Diffusion which uses the coherence of the image and a certain Partial Differential Equation to enhance the image. This image then undergoes a blind deconvolution restoration process which takes 
care of any image blurring across the edges. Finally, an image inpainting algorithm fills out those holes which still remain on the high resolution grid. The theory of all described techniques is treated in this section. The remaining of the section shows how to use these three methods in conjunction with each other to give the final enhanced image.

The fourth chapter gives the algorithmic details for all the different methods that have been used in alignment, interpolation and restoration. These algorithms can be used in conjunction with each other to give the desired super resolution algorithm. The different algorithms for the restoration part are decided depending on the nature and quality of the high resolution image obtained. If images are strongly blurred, then a blind deconvolution algorithm is implemented with an anisotropic diffusion algorithm. If the image has a large number of holes, then an inpainting algorithm does the trick. The next section gives the results of the algorithm followed by the conclusion and future work as the final two sections.

\subsection{Major Contributions}

Though our final aim in this thesis is the development of a robust super resolution algorithm, we have used the divide and conquer strategy in which we divide the main problem (i.e. super resolution) into multiple small problems such as the image alignment, image interpolation, and image restoration and then, attempted to solve each one of them separately. The algorithms and the results are then combined together to give the final algorithm. The contributions of this work can be summarized as,

\section{Image Alignment}

In image alignment, the basic problem is to align the multiple images and find out the affine parameters between them. This is achieved by a global process of phase correlation. The main advantages of this method are 
a. The simplicity by which the fourier shift theorem is used to estimate the translational parameters from the spatial domain and the rotational parameters from the log polar domain.

b. Being a generic process, it assumes no information about the nature of images to be aligned and thus can be used to any type of biometric and not just fingerprints.

c. This process is a global process and thus we avoid the problem of local deformations.

We have used an iterative strategy that estimates the translational and rotational parameters in a loop, such that the output of the translational module is fed to the rotational module and vice versa until we get a stable set of affine parameters. Thus the termination of the loop is obtained when the change in the estimated parameters is within an acceptable threshold.

\section{Image Interpolation}

The image interpolation problem basically refers to the problem of matching the multiple low resolution images onto one single high resolution pixel grid. For this we have to consider two points,

a. We have to extract information from all the copies and then map them onto the high resolution grid.

b. A decision has to be made in case of a discrepancy arising during cases in which information is available for a single pixel from two different sources (i.e. multiple copies).

For this we have followed the projection on to the convex sets (POCS) approach in which we define certain constraint sets such as finite energy, reference image and positivity to guarantee a convergence of the solution. This solution may not be 'the solution' but will give an estimate which is very near to the solution. The advantages of POCS are that it is a very simple process and we can include priori information about the image to be interpolated very easily. 
Introduction

\section{Image Restoration}

Image restoration is the process of enhancing the obtained raw image to give the final image. For this we use three different algorithms in conjunction with each other to give the final result.

While the remaining two modules assume no information about the image and will remain same for any biometric image, the image restoration module is specific to fingerprints. The main aim in developing this module was to enhance fingerprints and thus algorithms which will facilitate this process have been used. As stated earlier, we have used three different algorithms viz. the blind deconvolution, the anisotropic coherence enhancement and the image inpainting.

Though these modules have been developed earlier, in the signal processing and the computer vision societies, we have used these techniques to the field of biometrics for the first time. Also these methods were never used in conjunction with each other. We have developed an iterative feedback procedure which uses certain number of iterations of these individual processes to enhance fingerprints. 


\section{Chapter 2}

\section{Image Registration}

Image registration is the term given to the construction of a raw biometric high resolution image. This process can be further subdivided into the image alignment and the image interpolation method. Super resolution from a single low resolution, blurred image is an ill-posed problem. But, when a sequence of low resolution images is available, the complexity of the problem reduces slightly. As can be seen logically, the 3-D spatiotemporal sampling grid contains more information than any 2-D still image sampling grid. Multiframe super resolution methods exploit this additional information, contained in multiple images to reconstruct a high -resolution still image.

Thus super resolution from multiple copies becomes feasible due to the fact that, each low resolution observation from different copies potentially contains novel information about the desired high resolution image. Several multiframe methods exist which are in the form of two-stage interpolation-restoration algorithms. They are based on the premise that all pixels from available frames can be mapped back onto the reference frame, based on the affine transformation information, to obtain an up-sampled image.

The basic idea here is to estimate the motion vectors or affine parameters between the reference image and the remaining copies. With the use of this information, all the copies are correspondingly mapped on to the high resolution grid starting with a basic interpolation technique such as the bilinear interpolation. Now in subsequent iterations, this guess of the high resolution image is improved until we get the final image.

Thus as specified earlier, we can divide the process of registration into two main parts. The following sections describe both these methods along with the mathematical treatment. 


\subsection{Image Alignment}

Due to the motion of the camera, each image provides a different sampling of the same area. In order to restore the high-resolution information it is necessary to determine the relative motion between each low-resolution image. Once the motion is determined, the images are aligned and converted into a high-resolution frame. The relative motion between frames in a video sequence depends upon the frame rate of the sensor, and the velocity and direction of the subject's movements. It is assumed that all movements made by the subject are pure planar translations over any given area.

Adjacent frames in an image sequence will overlap with much of the same information. A registration algorithm is required to determine the extent of this overlap. The overlapping portions of each frame are aligned with one another, which provide the multiple samples of the same area necessary for super-resolution.

The majority of super-resolution algorithms depend upon relative motion, at the sub-pixel level, between the low-resolution images, though algorithms have been developed that do not [58]. Therefore, most super-resolution algorithms require a registration algorithm that can accurately determine relative sub-pixel motion between images. This thesis looks more specifically at an image sequence, where relative motion between image frames in the sequence is modeled as a simple planar translation and rotation.

Digital sensors rely on an array of photo sensitive elements, called charged-coupleddevices, to capture an image. Each charge-coupled-device, or CCD, corresponds directly to a pixel value in an image. The resolution of the image depends upon the number and spacing of CCDs in the array. Because the number and spacing of the CCDs is finite, high frequency information is lost. Resolution is also affected by the different parameters of the sensor; however this thesis is only concerned with restoring resolution lost by the CCD array. 
The most commonly used approach for sub pixel image registration consists of interpolating images prior to registration. Among this class of algorithms, we can notably mention: correlation interpolation [59, 60], intensity interpolation [60], phase correlation interpolation [60,61] and the geometric methods [62]. Obviously, the accuracy of the algorithm depends on the interpolation algorithm. This will be dealt with in the coset matching section.

Depending on the algorithm, alignment may be determined at the high or low resolution pixel level. If frames are aligned at the low-resolution level, a pixel from one frame will align with a specific pixel in other overlapping frames. Each pixel is considered a sample of a set of high-resolution pixels. The number of high-resolution pixels in a set depends upon the degree of resolution improvement.

If frames are aligned at the high-resolution level, pixels may not perfectly align from one frame to another. This is referred to as a sub-pixel shift. Once the registration algorithm has determined the alignment, each low-resolution frame is up-sampled into a high resolution estimate. The process of creating this estimate varies, depending upon the algorithm.

Methods that do not use interpolation for achieving sub pixel accuracy have been scarcer in the literature. Most of these rely on the differential properties of image features, whose main idea is to relate the difference between image frames to the intensity gradient of the reference image [63]. The difficulties involved with such methods are that they can be used only when the interframe displacements are very small, image gradient is only approximated using finite difference methods and finally these methods exhibit high sensitivity to noise due to noise amplifying feature of derivative operators.

The idea behind the phase correlation method is quite simple and is based on the Fourier shift theorem [64] and the fact that for two images with some degree of congruence, the signal power in their cross power spectrum is mostly concentrated in a coherent peak 
located at the point of registration while the noise power is distributed randomly in some incoherent peaks as can be seen in the Figure 9 below.

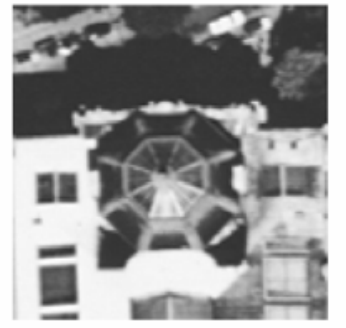

(a)

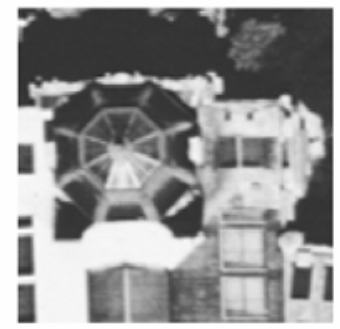

(b)

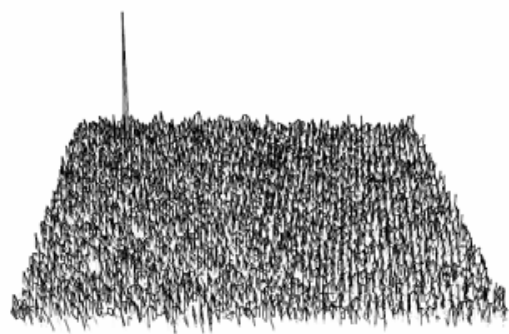

(c)

Figure 9: Correlation peak location for translated images only. [58]

Let $f_{1}(x, y)$ and $f_{2}(x, y)$ be two functions defined on $\mathbb{R}^{2}$

$F\left\{f_{1}(x, y)\right\}=F_{1}\left(\omega_{x}, \omega_{y}\right) \& \mathbb{F}\left\{f_{2}(x, y)\right\}=F_{2}\left(\omega_{x}, \omega_{y}\right)$

where, $F$ denotes the Fourier Transform.

Also, let $f_{2}(x, y)=f_{1}\left(x+x_{0}, y+y_{0}\right)$

Then by the fourier shift theorem,

$F_{2}\left(\omega_{x}, \omega_{y}\right)=F_{1}\left(\omega_{x}, \omega_{y}\right) e^{j\left(\omega_{x} x_{0}, \omega_{y} y_{0}\right)}$

Or equivalently,

$\frac{F_{2}\left(\omega_{x}, \omega_{y}\right) F_{1} *\left(\omega_{x}, \omega_{y}\right)}{\left|F_{2}\left(\omega_{x}, \omega_{y}\right) F_{1} *\left(\omega_{x}, \omega_{y}\right)\right|}=e^{j\left(\omega_{x} x_{0}, \omega_{y} y_{0}\right)}$

Where, * denotes the complex conjugate and the left hand side is referred to as the cross power spectrum of the two functions. It is now very easy to determine the $\mathrm{x}_{0}$ and $\mathrm{y}_{0}$ as the inverse Fourier transform of the right hand side is a Dirac delta function centered at $\left(\mathrm{x}_{0}\right.$, y0):

$\mathbb{F}^{-1}\left(\frac{F_{2}\left(\omega_{x}, \omega_{y}\right) F_{1} *\left(\omega_{x}, \omega_{y}\right)}{\left|F_{2}\left(\omega_{x}, \omega_{y}\right) F_{1} *\left(\omega_{x}, \omega_{y}\right)\right|}\right)=\mathbb{F}^{-1}\left(e^{j\left(\omega_{x} x_{0}+\omega_{y} y_{0}\right)}\right)=\delta\left(x_{0}, y_{0}\right)$ 


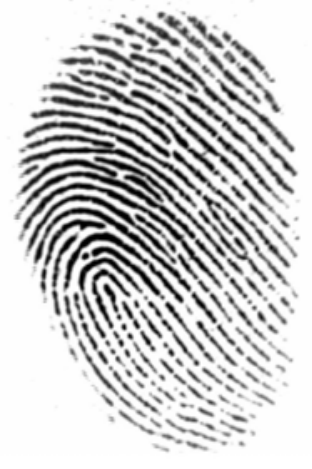

(a) Original image

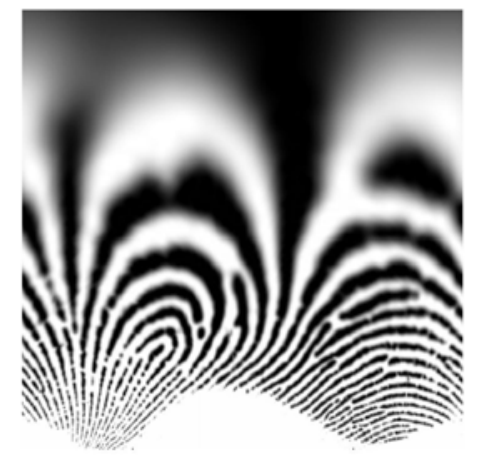

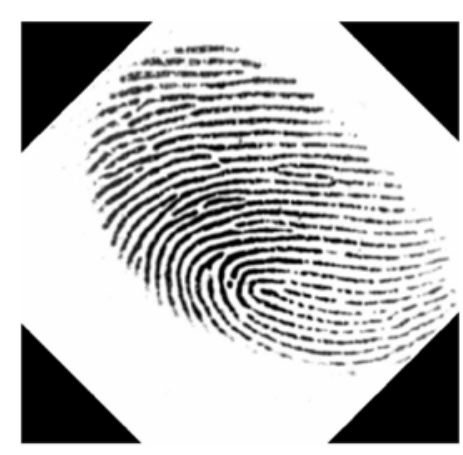

(b) rotated image

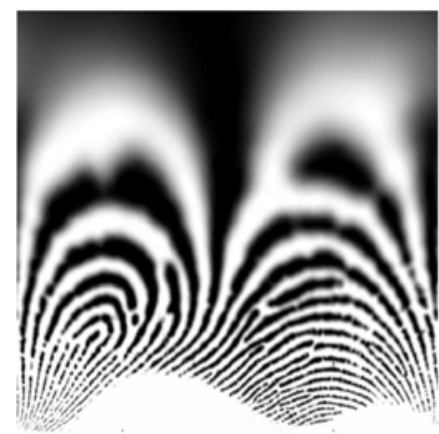

(c) and (d) log polar transforms of fingerprint images (a) and (b)

Figure 10: Fingerprint images and their log polar transforms

For the case of rotation and scaling, converting $\left|F_{2}\left(\omega_{x}, \omega_{y}\right) F_{1} *\left(\omega_{x}, \omega_{y}\right)\right|$ from rectangular coordinates to log-polar coordinates $(\log (\rho), \theta)$ makes is possible to represent both rotation and scaling as shifts (refer figure 10). But computing $(\log (\rho), \theta)$ for the original rectangular grid leads to points outside that grid. Thus, interpolation to find a value of $\left|F_{2}\left(\omega_{x}, \omega_{y}\right) F_{1} *\left(\omega_{x}, \omega_{y}\right)\right|$ on the desired grid is needed. A bicubic interpolation is used. Let $(\mathrm{x}, \mathrm{y})$ be a point related to the desired grid point $(\log (\rho), \theta)$,

$$
x=e^{\log (\rho)} \cos (\theta), y=e^{\log (\rho)} \sin (\theta)
$$


To find the new value $\mathrm{M}(\mathrm{x}, \mathrm{y})$, we look at the intensities $\mathrm{M}_{(\mathrm{j}, \mathrm{k})}, \mathrm{M}_{(\mathrm{j}+1, \mathrm{k})}, \mathrm{M}_{(\mathrm{j}, \mathrm{k}+1)}$ and $\mathrm{M}_{(\mathrm{j}+1, \mathrm{k}+1)}$ of four original grid points $(j, k),(j+1, k),(j, k+1)$, and $(j+1, k+1)$ surrounding $(x, y)$. Then, we can interpolate $\mathrm{M}(\mathrm{x}, \mathrm{y})$ as follows:

$\mathrm{M}(\mathrm{x}, \mathrm{y})=\mathrm{M}_{(\mathrm{j}, \mathrm{k})} \cdot(1-\mathrm{t}) \cdot(1-\mathrm{u})+\mathrm{M}_{(\mathrm{j}+1, \mathrm{k})} \cdot \mathrm{t} \cdot(1-\mathrm{u})+\mathrm{M}_{(\mathrm{j}, \mathrm{k}+1)} \cdot(1-\mathrm{t}) \cdot \mathrm{u}+\mathrm{M}_{(\mathrm{j}+1, \mathrm{k}+1)} \cdot \mathrm{t} \cdot \mathrm{u}$

Where, $\mathrm{t}$ is a fractional part of $\mathrm{x}$ and $\mathrm{u}$ is a fractional part of $\mathrm{y}$.

This method has a remarkable robustness to correlated noise and nonuniform time varying illumination, making it more appropriate in most practical applications compared to classical cross correlation method. Apart from using the convolution method, it can be easily shown that the method can handle blurred images since the blurring kernel would become a multiplicative factor in the spectral domain and would thus vanish automatically.

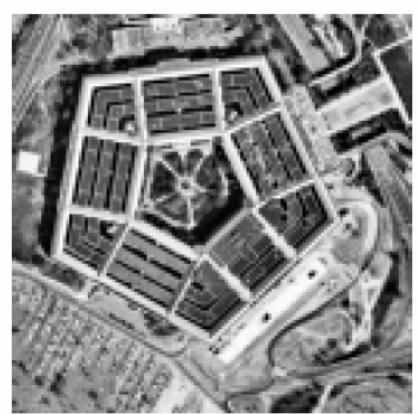

(a)

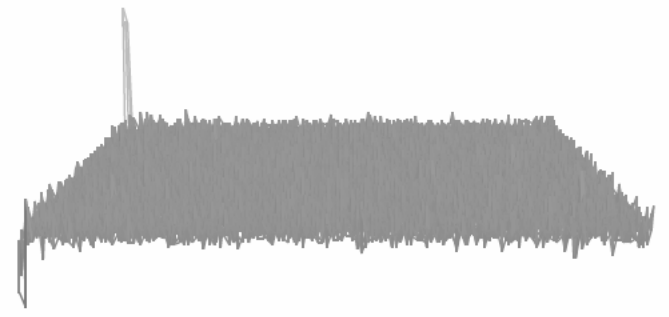

(c)

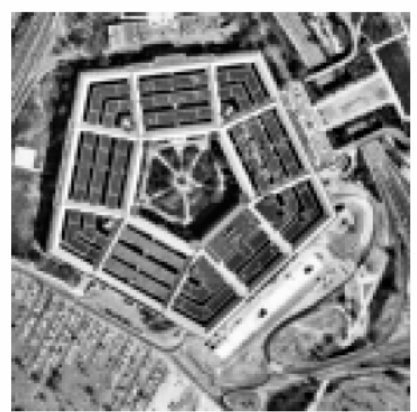

(b)

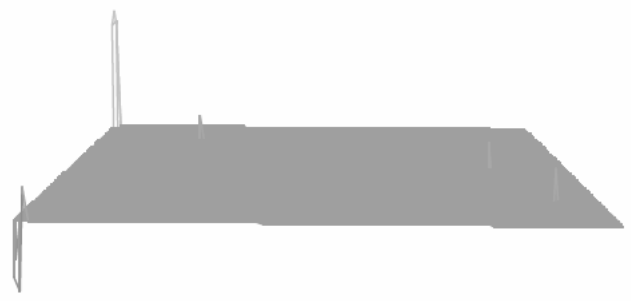

(d)

Figure 11: Images with sub pixel displacements and their cross power spectra with noise and after noise removal [58]

These techniques as can be seen can be used on the pixel level. The affine parameters that they will return are the translational and the rotational parameters at an integer level accuracy. But now the question remains, what if the alignment has to be on a sub pixel level. This is in conjunction with the fact that the aim behind this work is to increase the 
resolution of the image. So we need to align the images at the sub pixel domain so that when we interpolate the image to the desired resolution, the images are aligned in that resolution. Thus we need to have an algorithm in the sub pixel domain and then round it to the desired factor.

To formulate the problem, it is assumed that at some stage, two or more images with integer value displacements between them, have been down sampled, reducing the correspondence between them to sub pixel values. Thus for the sub pixel domain, the cross power spectrum of two down sampled images is examined. Down sampling can be seen as polyphase decomposition in the spectral domain.

Consider two images, $f(x, y)$ and $f\left(x+x_{0}, y+y_{0}\right)$ where the displacement vector $\left(x_{0}, y_{0}\right)$ is an integer valued vector. Also, as in the previous case, let,

$$
\mathbb{F}(f(x, y))=F\left(\omega_{x}, \omega_{y}\right) \text { and } \mathbb{F}\left(f\left(x+x_{0}, y+y_{0}\right)\right)=F\left(\omega_{x}, \omega_{y}\right) \exp \left(j\left(x+x_{0}, y+y_{0}\right)\right) \text {, }
$$

denote their discrete Fourier transforms. The corresponding discrete Fourier transforms after down sampling the images by factors $\mathrm{M}$ and $\mathrm{N}$ along $\mathrm{x}$ and $\mathrm{y}$ axes respectively, is given by, $[65,66]$.

$$
\begin{aligned}
& \phi_{1}\left(\omega_{x}^{\prime}, \omega_{y}^{\prime}\right)=\frac{1}{M N} \sum_{m=0}^{M-1} \sum_{n=0}^{N-1} F\left(\omega_{x}^{\prime}, \omega_{y}^{\prime}\right), \text { and } \\
& \phi_{2}\left(\omega_{x}^{\prime}, \omega_{y}^{\prime}\right)=\frac{1}{M N} \sum_{m=0}^{M-1} \sum_{n=0}^{N-1} F\left(\omega_{x}^{\prime}, \omega_{y}^{\prime}\right) \exp \left(j\left(\omega_{x}^{\prime} x_{0}, \omega_{y}^{\prime} y_{0}\right)\right)
\end{aligned}
$$

where, $\omega_{x}^{\prime}=\frac{\omega_{x}}{M}+\frac{2 \pi m}{M}, \omega_{y}^{\prime}=\frac{\omega_{y}}{N}+\frac{2 \pi n}{N}$, and $\phi_{1} \& \phi_{2}$ are the downsampled spectra.

Thus the cross power spectra of the down sampled images will be given by, 


$$
\begin{aligned}
\mathrm{C}\left(\omega_{x}^{\prime}, \omega_{y}^{\prime}\right) & =\frac{\frac{1}{M N} \sum_{m=0}^{M-1} \sum_{n=0}^{N-1} F\left(\omega_{x}^{\prime}, \omega_{y}^{\prime}\right) \exp \left(j\left(\omega_{x}^{\prime} x_{0}, \omega_{y}^{\prime} y_{0}\right)\right)}{\frac{1}{M N} \sum_{m=0}^{M-1} \sum_{n=0}^{N-1} F\left(\omega_{x}^{\prime}, \omega_{y}^{\prime}\right)} \\
& =\sum_{m=0}^{M-1} \sum_{n=0}^{N-1} H_{m n}\left(\omega_{x}^{\prime}, \omega_{y}^{\prime}\right) \exp \left(j\left(\omega_{x}^{\prime} x_{0}, \omega_{y}^{\prime} y_{0}\right)\right) \\
\text { where, } H_{m n}\left(\omega_{x}^{\prime}, \omega_{y}^{\prime}\right) & =\frac{F\left(\omega_{x}^{\prime}, \omega_{y}^{\prime}\right)}{\sum_{m=0}^{M-1} \sum_{n=0}^{N-1} F\left(\omega_{x}^{\prime}, \omega_{y}^{\prime}\right)}
\end{aligned}
$$

Thus, the cross power spectrum of two down sampled images is merely a polyphase decomposition of a filtered Dirac delta function. The filter can be identified by substituting back the values and rearranging the equation as,

$$
\begin{aligned}
& H_{m n}\left(\omega_{x}^{\prime}, \omega_{y}^{\prime}\right) \sum_{m=0}^{M-1} \sum_{n=0}^{N-1} F\left(\frac{\omega_{x}}{M}+\frac{2 \pi m}{M}, \frac{\omega_{y}}{N}+\frac{2 \pi n}{N}\right)=F\left(\frac{\omega_{x}}{M}+\frac{2 \pi k}{M}, \frac{\omega_{y}}{N}+\frac{2 \pi l}{N}\right), \\
& \text { where, } k \in[0 \ldots M-1] \text { and } l \in[0 \ldots N-1]
\end{aligned}
$$

Thus, obviously,

$$
H_{m n}\left(\omega_{x}^{\prime}, \omega_{y}^{\prime}\right)=\left\{\begin{array}{cc}
1 & \text { if }(m=k \& n=l) \\
0 & \text { otherwise }
\end{array}\right.
$$

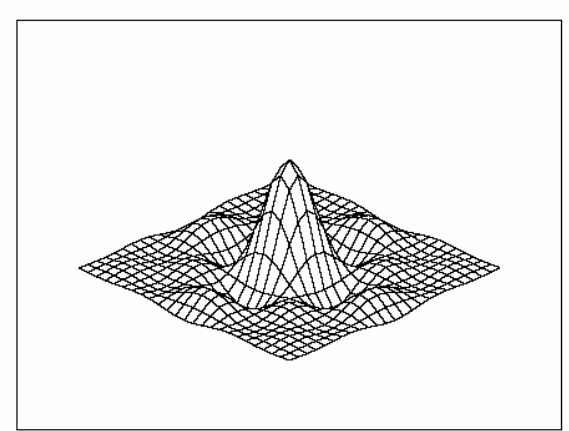

(a)

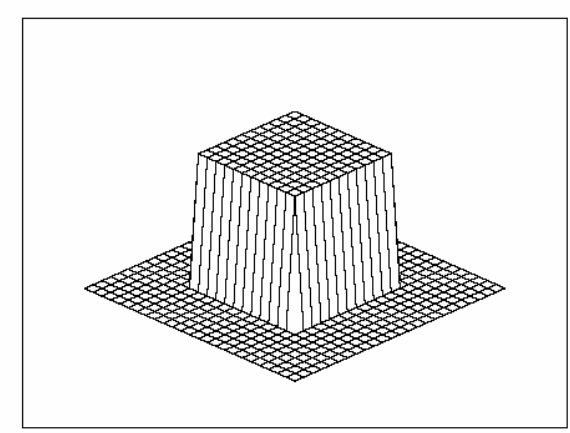

(b)

Figure 12: Spatial and frequency response of polyphase filter 
Summarizing, the cross power spectrum of two images is a Dirac delta function. However in the case of down sampled images it is shown that the cross power spectrum is a filtered Dirac delta function which has been down sampled. This filter has a $2 \mathrm{D}$ sinc spatial response (figure 12).

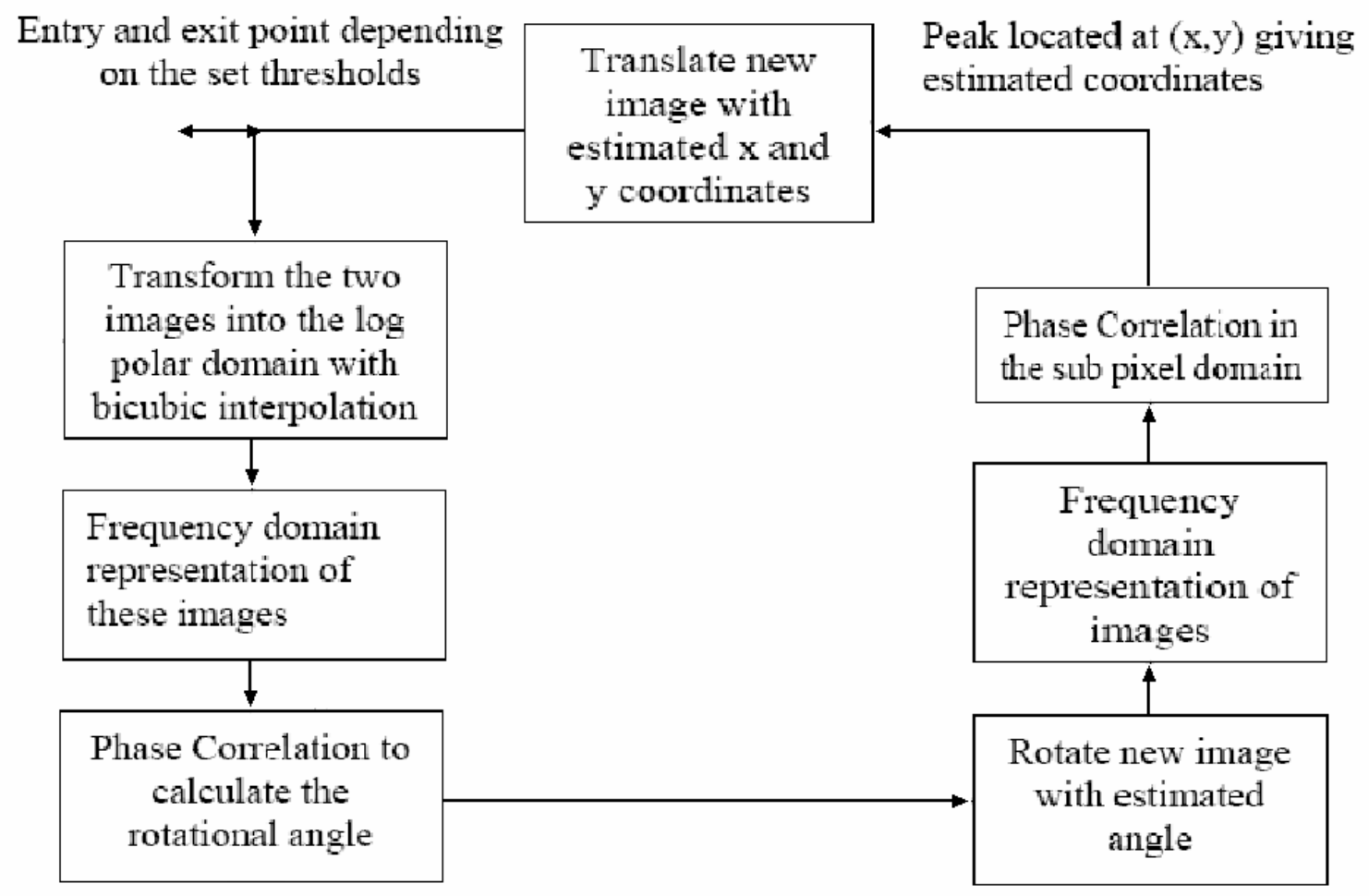

Peak at $\left(x^{\prime}, y^{\prime}\right), x^{\prime}$ gives the angle

Figure 13: Flowchart for estimating the affine parameters

Consider the situation where the signal power is mostly concentrated at pixels $(0,0),(1$, $0)$ and $(0,1)$. Then applying the above derived equation to the first two points yields,

$$
\begin{aligned}
& \frac{\sin \left(\pi x_{0}\right)}{\pi x_{0}} \frac{\sin \left(\pi y_{0}\right)}{\pi y_{0}}=c(0,0) \\
& \frac{\sin \left(\pi\left(M+x_{0}\right)\right)}{\pi\left(M+x_{0}\right)} \frac{\sin \left(\pi y_{0}\right)}{\pi y_{0}}=c(1,0)
\end{aligned}
$$


dividing both sides and rearranging, we get:

$$
\begin{aligned}
& \frac{\sin \left(\pi\left(M+x_{0}\right)\right)}{\pi\left(M+x_{0}\right)}=\frac{c(1,0)}{c(0,0)} \frac{\sin \left(\pi x_{0}\right)}{\pi x_{0}} \\
& \frac{ \pm \sin \left(\pi x_{0}\right)}{\pi\left(M+x_{0}\right)}=\frac{c(1,0)}{c(0,0)} \frac{\sin \left(\pi x_{0}\right)}{\pi x_{0}}
\end{aligned}
$$

$$
\text { Simplifying, } \frac{x_{0}}{M}=\frac{c(1,0)}{ \pm c(0,0)-c(1,0)}
$$

Thus we see that to find the sub pixel displacement in $\mathrm{x}$ direction, no knowledge of the down sampling rate $M$ is required. Note that two solutions will be obtained. This ambiguity is due to the isotropic form of $\mathrm{c}(\mathrm{x}, \mathrm{y})$ and hence only one of these is the right solution. Similarly we can determine the sub pixel displacement in the y direction.

\subsection{Image Interpolation}

One of the prominent approaches to super-resolution reconstruction is based on the method of projection onto convex sets. In this formulation, constraint sets are defined which limit the feasible solution space of the super-resolution reconstruction. Constraints

are defined as convex sets in the vector space $R^{N_{1} x N_{2}}$, which represents the space containing all possible super-resolution reconstructions. Sets that represent desirable characteristics of the solution are defined, such as positivity, bounded energy, fidelity to data, smoothness and so on. The solution space of the super resolution reconstruction problem is thus the intersection of the convex constraint sets.

Projection onto convex sets (POCS) refers to an iterative procedure which, given any point in the vector space, locates a point which satisfies all the convex constraint sets. A set $\Omega$ is said to be convex if, for any two points $x \in \Omega, y \in \Omega$, we have,

$$
a x+(1-a) y \in \Omega, \forall 0 \leq a \leq 1
$$


The following are the examples of the convex sets:

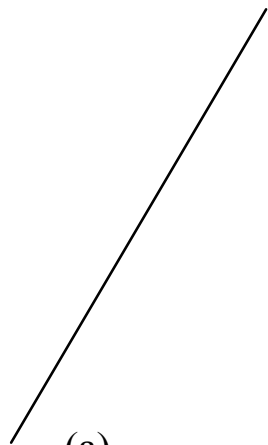

(a)

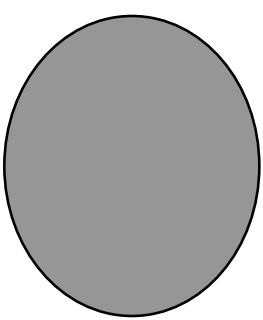

(b)

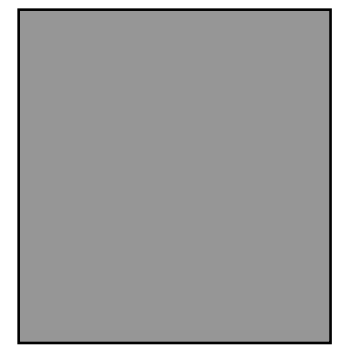

(c)

Figure 14: Examples of convex sets

Similarly, the following are the examples of non-convex sets:

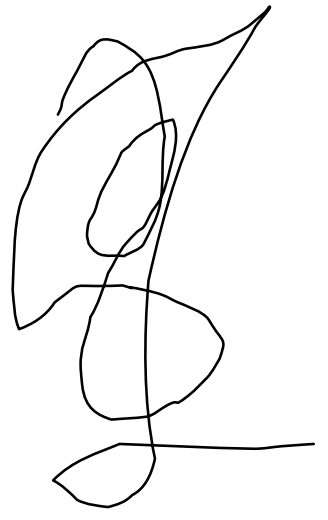

(a)

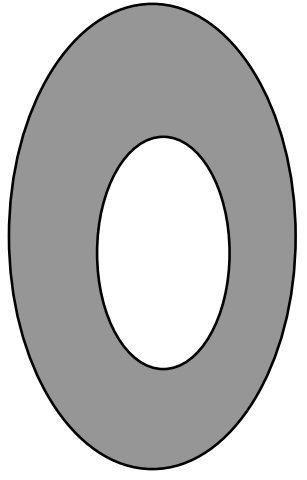

(b)

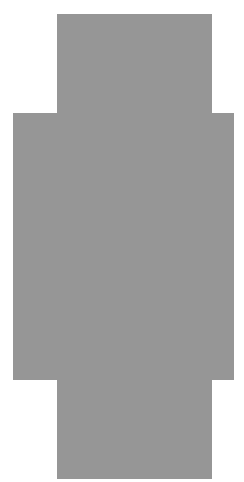

(c)

Figure 15: Examples of non-convex sets

The projection operator for the convex set theory is defined as:

$$
g=P f=\left\{x \in C \mid\|x-f\|=\min _{x \in C}\|x-f\|\right\}
$$




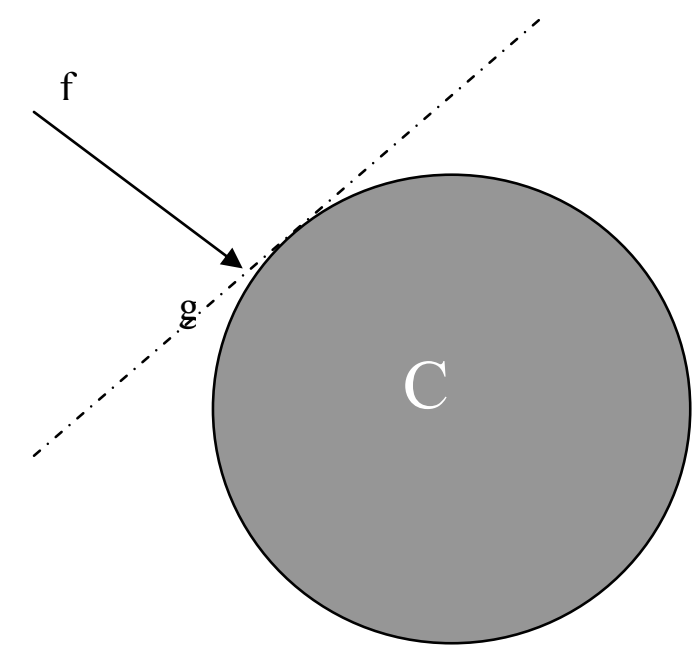

Figure 16: Projection onto the convex set

Where, $\mathrm{g}$ is the projection onto the convex set as shown in the figure. In simple words, the projection of $\mathrm{f}$ onto a convex set $\mathrm{C}$ is the element in $\mathrm{C}$ that is closest to $\mathrm{f}$ in terms of Euclidean distance.

Given k convex constraint sets in $R^{N_{1} X N_{2}}$ such that the intersection of the sets is nonempty, POCS projects a point in the vector space onto each constraint set, and repeating until a point is reached which is in the intersection of the $\mathrm{k}$ sets. It can be shown that provided the constraint sets are convex that this iteration converges. A detailed theoretical discussion of the POCS method may be found in [67]. Discussions on the use of POCS techniques in image restoration are presented in $[68,69]$.

POCS has attracted much attention in recent years in a multitude of image reconstruction and restoration applications. Three reasons for this stand out [71]:

\section{Simplicity}

POCS is very intuitive and generally simple to implement. The only potential source of difficulty is the determination of the projection operators.

\section{Flexible Spatial Domain Observation Model}

Because the POCS method is typically formulate in the spatial domain, very general motion and observation models may be used. The complexity of the motion and observation model has little impact of the POCS solution procedure. 


\section{Powerful inclusion of a priori information}

Perhaps the most useful aspect of the POCS formulation is the ease with which apriori information may be included. It is generally simple to define convex constraint sets which incorporate desired solution characteristics. These sets may impose restrictions such as positivity or bounded energy which is difficult to represent in terms of cost functionals.

The fundamental idea of POCS for interpolation was proposed by Stark and Oskoui [70] to super-resolution image reconstruction problems. In particular, the paper addresses the scanning linear array problem as well as the problem of restoring a super-resolution image from multiple plane array images. The proposed image model is described below. The mathematics for the case of a time invariant image is treated. It will be clear how the method is extended to the case of time varying imagery with multiple image acquisitions.

The optical system projects an image $\mathrm{f}(\mathrm{x}, \mathrm{y})$ onto the image sensor array which is assumed to be a regular array of $M_{1}$ by $M_{2}$ sensor elements. The output $y_{i}$ of the $i^{\text {th }}$ detector in the sensor array with spatial response characteristic $\sigma_{i}(x, y)$ is given by,

$$
y_{i}=\iint_{-\infty}^{\infty} f(x, y) \sigma_{i}(x, y), 1 \leq i \leq M_{1} M_{2}
$$

This integration over the continuous spatial variables $(\mathrm{x}, \mathrm{y})$ may be discretized on the super resolution reconstruction grid $\left[\mathrm{n}_{1}, \mathrm{n}_{2}\right]$ yielding,

$$
y_{i}=\sum_{n_{1}} \sum_{n_{2}} f\left[n_{1}, n_{2}\right] \sigma_{i}\left[n_{1}, n_{2}\right], 1 \leq n_{1} \leq N_{1}, 1 \leq n_{2} \leq N_{2}
$$

Where $\mathrm{N}_{1}>\mathrm{M}_{1}, \mathrm{~N}_{2}>\mathrm{M}_{2}$ are the dimensions of the super-resolution reconstruction array. The $\mathrm{i}^{\text {th }}$ detector spatial response characteristic $\sigma_{i}(x, y)$ is discretized to yield $\sigma_{i}\left[n_{1}, n_{2}\right]$ which is the fractional area of the super-resolution pixel $\left[n_{1}, n_{2}\right]$ contained within the response region of the $i^{\text {th }}$ low-resolution detector. This assumes a uniform, unity response of the detector over its response region. 
In particular, $\sigma_{i}\left[n_{1}, n_{2}\right]=\left\{\begin{array}{rr}0 & \text { if SR pixel }\left[n_{1}, n_{2}\right] \text { is completely outside of } \mathrm{i}^{\text {th }} \text { detector response region } \\ 1 & \text { if SR pixel }\left[n_{1}, n_{2}\right] \text { is completely within of } \mathrm{i}^{\text {th }} \text { detector response region } \\ r_{i} & \left(0<\mathrm{r}_{\mathrm{i}}<1\right) \text { if SR pixel }\left[n_{1}, n_{2}\right] \text { is partially within of } \mathrm{i}^{\text {th }} \text { detector response region }\end{array}\right.$

We have,

$y_{i}=\sigma_{i}^{T} f$

Let, $C_{i}=\left\{f: \sigma_{i}^{T} f=y_{i}\right\}, 1 \leq i \leq M_{1} M_{2}$

The set $\mathrm{C}_{\mathrm{i}}$, is the set of all discretized super-resolution images for which the response of the $\mathrm{i}^{\text {th }}$ sensor in the detector array is $y_{i}$, the observed value. The set $C_{i}$ is defined for each pixel observation $\mathrm{i}, 1 \leq i \leq M_{1} M_{2}$ and thus there are a total of $\mathrm{M}_{1} \mathrm{M}_{2}$ such sets for a single image observation. These sets place a constraint on the possible values which may be assumed by the solution $\mathrm{f}$. Noticing that the constraints on $\mathrm{f}$ are linear, it is in principle possible to obtain a sufficient number of equations, so that a solution for $\mathrm{f}$ may be found by matrix inversion.

For reconstruction of a super-resolution image with $\mathrm{N}_{1} \mathrm{~N}_{2}$ pixels by matrix inversion, $\mathrm{N}_{1} \mathrm{~N}_{2}$ independent observation equations are required. Notice immediately that since $\mathrm{N}_{1}>\mathrm{M}_{1}, \mathrm{~N}_{2}>\mathrm{M}_{2}$, data from more than one image acquisition is required to obtain a sufficient number of equations to enable solution by matrix inversion. Indeed the requirement to obtain $\mathrm{N}_{1} \mathrm{~N}_{2}$ independent equations implies that novel information must be present data obtained in the acquisition of the additional images. This novel information is present in small differences in the acquired images usually resulting from scene or camera motion. In practice however, direct matrix is infeasible.

These additional constraints augment the under-determined system of equations derived for a single image so that the system approaches the fully-determined case. The collection of sets $\mathrm{C}_{\mathrm{i}}$ is often called data consistency constraints and in this case can be shown to be closed and convex. 
This allows the definition of a projection operator $\mathrm{P}_{\mathrm{i}}$ as follows:

$$
P_{i} f=\left\{\begin{array}{cc}
f & \sigma_{i}^{T} f=y_{i} \\
f+\frac{y_{i}-\sigma_{i}^{T} f}{\sigma_{i}^{T} \sigma_{i}} & \text { otherwise }
\end{array}\right.
$$

$\mathrm{P}_{\mathrm{i}} \mathrm{f}$ is the projection of the point $\mathrm{f}$ onto the set $\mathrm{C}_{\mathrm{i}}$. In the POCS formulation, an initial guess $f^{(0)}$ for the super-resolution image is projected onto each the constraint sets $\mathrm{C}_{\mathrm{i}}$ to ensure consistency with each measured datum $\mathrm{y}_{\mathrm{i}}$ and where $\mathrm{K}$ is the number of pixel measurements.

$$
f^{(n+1)}=P_{1} P_{2} P_{3} \ldots P_{K} f^{(n)}
$$

This set of projections is applied repeatedly to yield an updated estimate of the superresolution image. Closedness and convexity of the constraint sets ensure convergence of the iteration to some point satisfying all the constraint sets. It is imperative to note however, that this point is in general non-unique, but is a point on the surface of the convex polytope defined by the intersection of the constraint sets. The solution is in general dependent on the initial guess.

We have used the concept of alternating projections to accommodate all the different constraints. This is given by the POCS theorem: If $\mathrm{C} 1, \ldots, \mathrm{Ck}$ are convex sets, then alternating projection $\mathrm{P} 1, \ldots, \mathrm{Pk}$ will converge to the intersection of $\mathrm{C} 1, \ldots, \mathrm{Ck}$ if it is not empty. This can be illustrated by the following example.

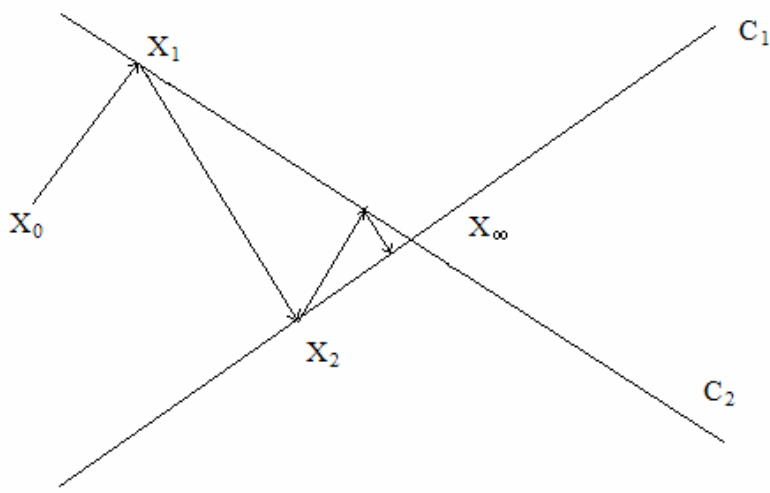

Figure 17: Alternating projections 
In addition to the data consistence constraints, additional constraints which represent apriori knowledge of the form of the solution may be included. In particular the range of values in the solution image may be constrained to the set $\mathrm{C}_{\mathrm{A}}$,

$$
C_{A}=\left\{f: \alpha \leq f_{i} \leq \beta, \alpha<\beta\right\}
$$

Another possibility is to ensure that the solution be contained in a set of solutions with bounded energy as:

$$
C_{E}=\{f:\|f\| \leq E\}
$$

Or if the solution is known to be similar to some reference image $f_{R}$ then the solution may be constrained to the sets,

$$
C_{R}=\left\{f:\left\|f-f_{R}\right\| \leq \varepsilon_{R}\right\}
$$

Bounded support of the solution may also be imposed by defining a set of points A for which the solution is required to be zero, such as

$$
C_{s}=\left\{f: f_{i}=0 \text { for } \mathrm{i} \in \mathrm{A}\right\}
$$

Additional constraints on the solution may be defined in a similar manner. The inclusion of prior knowledge in this fashion constrains the solution space thus enabling robust performance in the presence of noise, inconsistent data or missing data.

There are, however, several drawbacks to this approach. One of them is that the proposed observation model does not incorporate noise. The observation model for the $\mathrm{k}^{\text {th }}$ single observed image is of the form, [72]

$$
\begin{aligned}
& y_{k}=H_{k} f \\
& H_{k}=\left[\sigma_{1_{k}}^{T} \sigma_{2_{k}}^{T} \ldots \sigma_{M_{1_{k}} M_{2_{k}}}^{T}\right]^{T}
\end{aligned}
$$

Which, may be written in the case of $p$ image observations as,

$$
\begin{aligned}
& Y=H f \\
& Y=\left[y_{1}^{T} y_{2}^{T} \ldots y_{k}^{T} \ldots y_{p}^{T}\right]^{T} \\
& H=\left[H_{1}^{T} H_{2}^{T} \ldots H_{k}^{T} \ldots H_{p}^{T}\right]^{T}
\end{aligned}
$$


Tekalp, Ozkan and Sezan [72] propose three different super-resolution reconstruction approaches. We have concentrated on the POCS based approach which extends the earlier work by Stark and Oskoui [70]. The other proposed approaches, which are a frequency domain formulation and an interpolation/restoration method. The primary contribution of the POCS method is an observation model which includes noise. In particular, a single image observation model of the form is proposed,

$$
\mathrm{y}=\mathrm{Hf}+\mathrm{n}
$$

Where the system matrix $\mathrm{H}$ includes the effects the sensor PSF. Only global translational motion is modeled. In order to account for the observation noise, the data consistency constraint for the $\mathrm{i}^{\text {th }}$ pixel observation [70] is modified to the form,

$$
C_{i}=\left\{f:\left|r_{i}\right|<\delta_{0}\right\}
$$

Where, $r_{i}$ is the $i^{\text {th }}$ element of the residual,

$$
\mathrm{r}=\mathrm{y}-\mathrm{Hf}
$$

Noticing that

$$
\mathrm{y}-\mathrm{Hf}=\mathrm{n},
$$

It is clear that the residual $\mathrm{r}$ is nothing more than the observation noise $\mathrm{n}$ and so $\delta_{0}$ represent a confidence in the observation and may be set according to the noise statistics.

Patti, Sezan and Tekalp address the shortcomings of this and so their paper utilizes the same general observation model $\mathrm{y}=\mathrm{Hf}+\mathrm{n}$ and a POCS formulation for the solution of these equations. The primary development in this paper is the motion model included in the system matrix $\mathrm{H}$ which departs significantly from previous work.

Most significantly, the matrix $\mathrm{H}$ incorporates a space varying PSF (SVPSF) which models the degradations caused by sampling with a low-resolution sensor, as well as the effects of relative motion between the sensor and the scene (which includes blurring due to a non-zero aperture time). The SVPSF results from three modeling stages; first the blur caused by the relative motion between the scene and the sensor during the aperture time; next the effects of the physical dimensions and response characteristics of the low 
resolution sensors; and finally the effects of sampling by the sensor array. The combined model defines the system matrix $\mathrm{H}$.

We provide a POCS based framework for super-resolution reconstruction which accounts for aliasing, sensor blur, motion blur and additive noise. It is interesting to note that spatial blurring due to motion occurring during the aperture time had not been addressed prior to this work. Additionally this work relaxed the constraint that blur and noise affecting each acquired image be LSI.

In addition to their work addressing the super-resolution reconstruction problem, Patti, Sezan and Tekalp have also published in the related but not identical problem of standards conversion, [73], in which the objective is robust spatial and temporal resampling of a video signal in one spatiotemporal sampling lattice to some other lattice. This work extends $[72,74]$ to include an arbitrary input spatio-temporal sampling lattice.

The form of the discrete observation model is unchanged from the SVPSF model proposed in [74]. A POCS formulation identical to that found in [74] is utilized for reconstruction. The problem is formulated as a problem of solving a set of simultaneous equations in the space domain. It is clear from the above theory that we can obtain MxM equations for each frame, for a total of $L^{2} x M x M$ equations in $N x N$ unknowns, $f(k, l)$. Given that $\mathrm{N}=\mathrm{LxM}$, we have a set of simultaneous equations is as many unknowns. One point worth mentioning is that in order for the equations to be linearly independent all displacements between the successive frames must be at sub pixel amounts.

In order to account for the observation noise, the following closed, convex constraints (one for each observed low resolution image pixel at each frame) 


$$
\begin{aligned}
& C_{m, n: i}=\left\{f(k, l):\left|r_{i}^{(f)}(m, n)\right| \leq \delta_{0}\right\}, \\
& 0 \leq m, n \leq M-1, i=1, \ldots, L^{2}, \\
& \text { where, } \\
& r_{i}^{(f)}(m, n) \doteq g_{i}(m, n)-\sum_{k=0}^{N-1} \sum_{l=0}^{N-1} f(k, l) h_{i}(m, n ; k, l) \\
& \doteq \rightarrow \text { is defined as. }
\end{aligned}
$$

and $\delta_{0}$ represents the confidence that is there in the observation and is equal to $c \sigma_{v}$, where $\sigma_{v}$ is the standard deviation of the noise and $\mathrm{c}>0$ is determined by an appropriate statistical confidence bound.

The projection $y(k, l) \doteq P_{m, n ; i}[x(k, l)]$ of an arbitrary x $(\mathrm{k}, 1)$ onto $C_{m, n ; i}$ is defined as, [75]:

$$
P_{m, n ; i}[x(k, l)]=\left\{\begin{array}{cc}
x(k, l)+\frac{r_{i}^{(x)}(m, n)-\delta_{0}}{\sum_{o} \sum_{p} h_{i}^{2}(m, n ; o, p)} h_{i}(m, n ; k, l) & \text { if } \mathrm{r}_{\mathrm{i}}^{(\mathrm{x})}(m, n)>\delta_{0} \\
x(k, l) & \text { if }-\delta_{0}<\mathrm{r}_{\mathrm{i}}^{(\mathrm{x})}(m, n)<\delta_{0} \\
x(k, l)+\frac{r_{i}^{(x)}(m, n)+\delta_{0}}{\sum_{o} \sum_{p} h_{i}^{2}(m, n ; o, p)} h_{i}(m, n ; k, l) & \text { if } \mathrm{r}_{\mathrm{i}}^{(\mathrm{x})}(m, n)<-\delta_{0}
\end{array}\right.
$$

Along with this we have also used other constraints such as finite energy, positivity and limited support to improve the results. 


\section{Chapter 3}

\section{Enhancement Techniques}

The image thus constructed on the high resolution grid using multiple copies of the same image with a lower resolution needs to undergo an enhancement process to obtain the final high resolution image required. The original raw high resolution image has a certain amount of image blurring due to the estimation of the high frequency component which is lost due to the image capturing process of the sensor.

The problem of image restoration is an ill-posed problem. That means a small variation in the data (due to noise) can cause large variation in the solution. Hence prior knowledge of the nature of the original image and noise is incorporated in the restoration process. Given the above constructed super resolution image, we seek a restoration system that would take this image and restore the original image as close as possible. The block diagram of the degradation model is shown below (Figure 18).

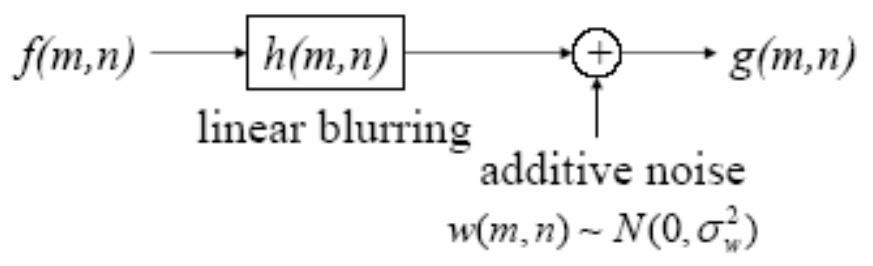

Figure 18: An image degradation model

The idea is to basically invert the effect of distortion and obtain an image, which is close to the original. There are many techniques for restoration of images that can be grouped into the following categories.

1) Deterministic - stochastic

2) Recursive - nonrecursive - iterative

3) Linear - nonlinear

4) Spatial domain - frequency domain

5) Adaptive - nonadaptive 
We have predominantly used three enhancement techniques to tune the high resolution image. The processes are a coherence enhancing anisotropic diffusion [100], a novel blind deconvolution method [76] and an image inpainting algorithm given by Dr. Sapiro [48]. The following section explains these three techniques in brief and the way we have used them for the enhancement.

\subsection{Blind Deconvolution}

Image restoration is an important and difficult task in image processing. The goal of restoration is to reconstruct or recover an image that has been degraded by using some a priori knowledge of the degradation phenomenon and true image. Most restoration techniques model the degradation process and attempt to apply an inverse procedure to obtain an approximation of the original image. The difficulty of image restoration arises largely because of the following [76]:

1. The degradation process is often unknown and must be estimated from the blurred image characteristics.

2. Even when the degradation is known, the inversion process required may not be unique or may be ill-conditioned.

3. The actual noise value at each pixel is unknown and must be estimated using the statistics of the noise or degraded image.

In many situations, the PSF is unknown and little can be assumed about the original image. Therefore, the majority of existing image restoration techniques is not applicable for solving this type of problem. The process of simultaneously estimating the PSF (or its inverse) and restoring an unknown image is known as blind image restoration. The blind deconvolution problem for images may be expressed as follows:

Given a blurred image, find an estimate of the true image using partial or no information about the PSF and true image. 
In practice, some a priori information is required to successfully restore the image. The partial information available is specific to each imaging applications, so many diverse techniques for blind deconvolution of images have been proposed. The challenge is to design a method that exhibits the most appropriate compromise among computation complexity, reliability, and robustness to noise for a given application.

The methods developed for blind deconvolution of images apply to the restoration of grey-scale images. They can be grouped into six major classes based on their assumptions about the true image and blur. Various algorithms and implementations for each class exist. Figure 19 provides a classification map of existing techniques. The basis of comparison is broken down into four categories:

- Reliability of solution: the convergence and uniqueness properties of the solution

- Computational complexity: the computational processing required for proper restoration

- Portability of algorithm: the potential for using the algorithm for many different applications

- Robustness to noise: reliability of the algorithm in the presence of additive noise

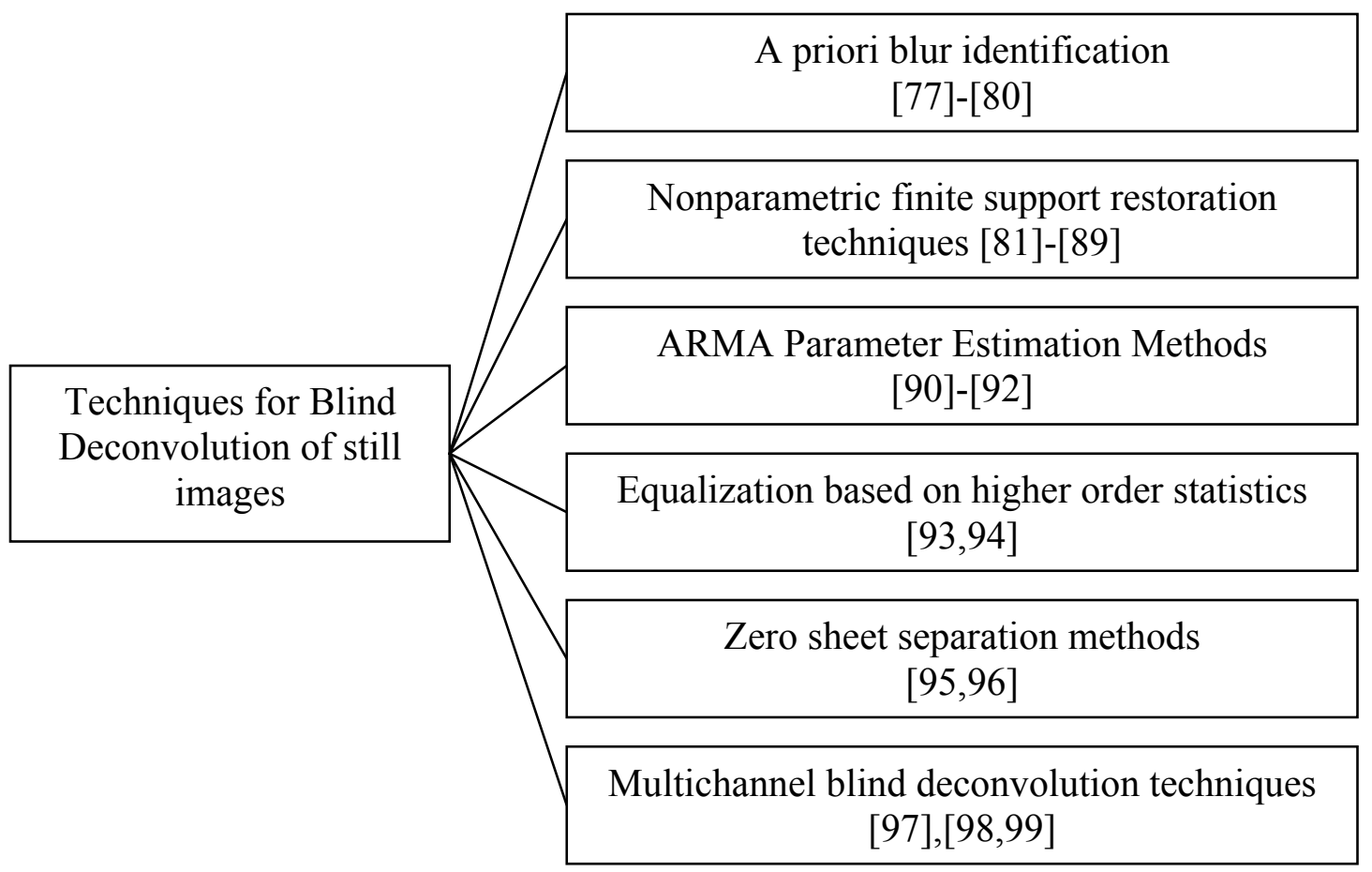

Figure 19: Techniques for blind image deconvolution 
The algorithm which has been used is the nonnegativity and support constraints recursive inverse filtering (NASRIF) algorithm which assumes that the point spread blurring function (PSF) is absolutely summable, i.e. $\sum_{\forall x, y}|h(x, y)|<\infty$, and that it has an inverse $\mathrm{h}^{-1}$ $(\mathrm{x}, \mathrm{y})$ which is also absolutely summable. As there are no other constraints on the PSF, it does not require the PSF to be of known finite extent. The NASRIF technique is shown in Figure 20. It consists of a variable FIR filter $\mathrm{u}(\mathrm{x}, \mathrm{y})$ with the blurred image $\mathrm{g}(\mathrm{x}, \mathrm{y})$ as the input. The output of the filter represents an estimate of the true image $\tilde{f}(x, y)$. This estimate is passed through a nonlinear filter, which uses a non-expansive mapping to project the estimated image into the space representing the known characteristics of the true image. The difference between the projected image $\tilde{f}_{N L}(x, y)$ and $\tilde{f}(x, y)$ is used as the error signal to update the variable filter $u(x, y)$.

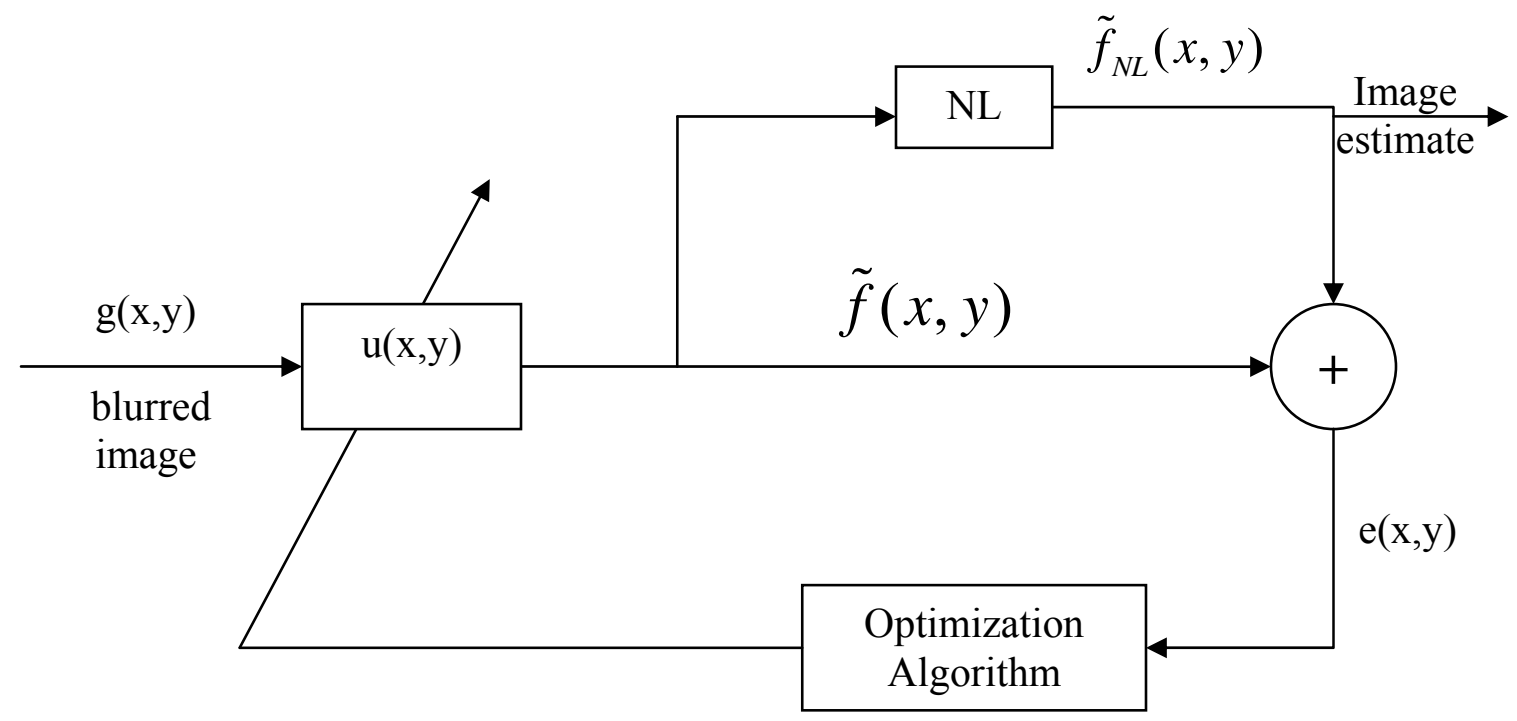

Figure 20: Feedback system diagram for blind image deconvolution

Under the assumption that the image is non negative with known support, the NL block represents the projection of the estimated image onto the set of images that are non negative with given finite support. Thus, the negative pixel values within the region of support must be zero, and the pixel values outside the region of support are the background grey level LB. Either the nonnegativity constraint or support constraint or 
both can be used for restoration. A cost function thus defined for the particular scenario is:

$$
\begin{aligned}
J(u) & =\sum_{\forall(x, y)} e^{2}(x, y)+\gamma\left[\sum_{\forall(x, y)} u(s, y)-1\right]^{2} \\
& =\sum_{(x, y) \in D_{\text {sup }}} \tilde{f}^{2}(x, y)\left[\frac{1-\operatorname{sgn}(\tilde{f}(x, y))}{2}\right]^{2}+\sum_{(x, y) \in \bar{D}_{\text {sup }}}\left[\tilde{f}(x, y)-L_{B}\right]^{2}+\gamma\left[\sum_{\forall(x, y)} u(s, y)-1\right]^{2}
\end{aligned}
$$

A detailed algorithm for the same is described in the $4^{\text {th }}$ section. The following figure will demonstrate the effectiveness of the algorithm for deblurring (figure 21).

(a) True Image

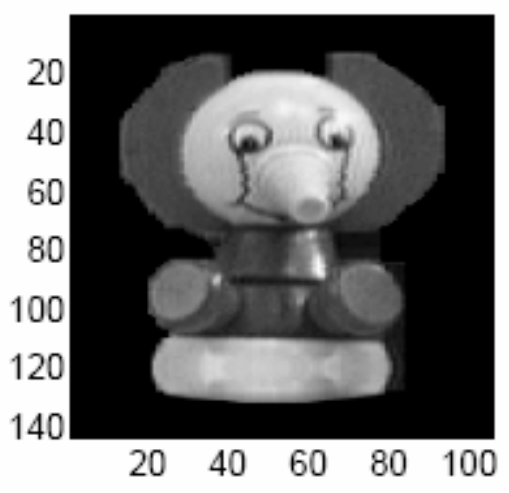

(c) NAS-RIF Restoration, $1=100$

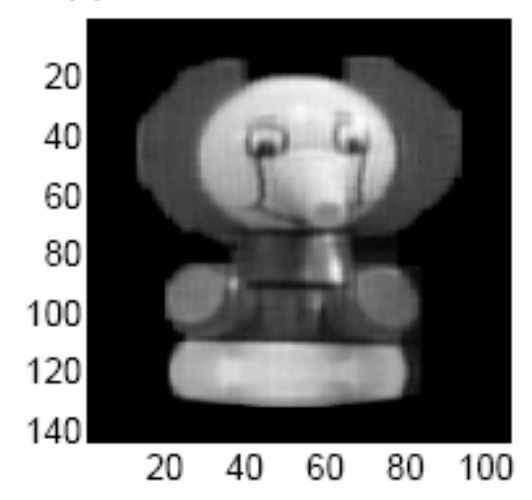

(b) Blurred Image

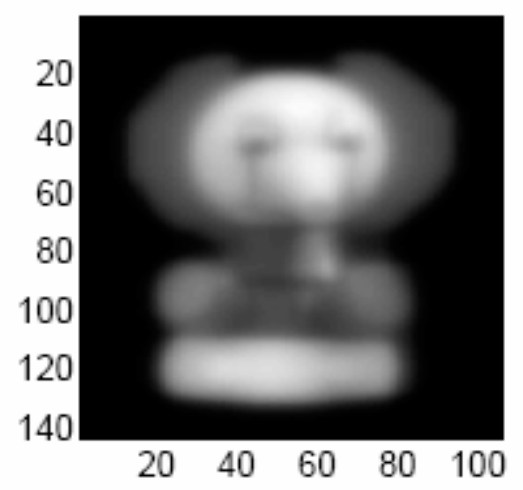

(d) Mean Square Error

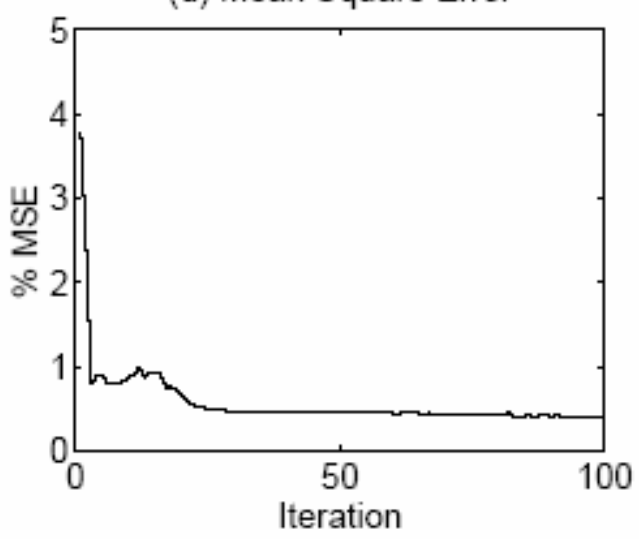

Figure 21: Results of the blind deconvolution algorithm and the MSE. [76] 


\subsection{Anisotropic Coherence Enhancement}

Starting from Perona and Malik's pioneering work in 1987 [101], non linear diffusion filtering has become a popular tool for improved sub sampling algorithms [102], post processing of fluctuating numerical data [103], blind image restoration [104], computer aided quality control $[105,106]$ and segmentation of textures $[107,108]$. The nonlinear diffusion filters regard the original image as the initial state of diffusion process that adapts itself to the evolving image. Different adaptation strategies provide different ways to include a priori knowledge into the evolution.

In order to avoid the blurring and localization problems of linear diffusion filtering, Perona and Malik proposed a nonlinear diffusion method. Their nonuniform process (which was rephrased as anisotropic) reduces the diffusivity at those locations that have a larger likelihood to be edges or those which have a high gradient. In 1992, Catte, Lions, Morel and Coll [109] proposed a regularization of the Perona Malik process that has a unique solution, and which is even infinitely times differentiable. Besides this theoretical advantage, their modification is more robust under noise. A noticeable drawback of this method was the fact that even though noise within a region is eliminated, at the edges, the diffusion tensor is large and the diffusion is inhibited. Thus the regularization is not optimal in the vicinity of noisy edges.

An anisotropic diffusion filter for edge enhancing diffusion does not only take into account the contrast of an edge, but also its direction. This can be achieved by constructing the orthonormal system of eigenvectors $v_{1}, v_{2}$ of the diffusion tensor D such that $v_{1} \| \nabla u_{\sigma} \& v_{2} \perp \nabla u_{\sigma}$. In order to prefer smoothing along the edge to smoothing across it, one can choose the corresponding eigenvalues $\lambda_{1} \& \lambda_{2}$ as [103].

$$
\begin{aligned}
& \lambda_{1} \doteq D\left(\left|\nabla u_{\sigma}\right|^{2}\right) \\
& \lambda_{2} \doteq 1
\end{aligned}
$$

Another motivation for introducing anisotropy into diffusion process arises from the wish to process 1-D features such as line-like structures. For this purpose one needs more 
sophisticated structure descriptors than $\nabla u_{\sigma}$. A good descriptor for local orientation is the structure tensor $J_{\rho}\left(\nabla u_{\sigma}\right) \doteq K_{\rho} *\left(\nabla u_{\sigma} \nabla u_{\sigma}^{T}\right)$. The component wise convolution with the Gaussian $K_{\rho}$ averages orientation information over an integration scale $\rho$. Because $J_{\rho}$ is a symmetric positive semi definite matrix, there exists an orthonormal basis of eigenvectors $v_{1}, v_{2}$ with corresponding eigenvalues $\mu_{1} \geq \mu_{2} \geq 0$. The eigenvalues measure the average contrast in the eigendirections within a scale $\rho$. Therefore, $v_{1}$ is the orientation with the highest gray value fluctuations and $v_{2}$ gives the preferred local orientation, the coherence direction. $\left(\mu_{1}-\mu_{2}\right)^{2}$ is a measure of the local coherence? For coherence enhancement, the image should be smoothed mainly along the coherence direction $v_{2}$ with diffusivity $\lambda_{2}$ that increases with respect to the coherence $\left(\mu_{1}-\mu_{2}\right)^{2}$. This can be accomplished by designing D such that it possesses the same eigenvectors $v_{1}, v_{2}$ as $J_{\rho}$ and choosing its corresponding eigenvalues as,

$$
\begin{array}{lc}
\lambda_{1} \doteq \alpha & \begin{array}{cc}
\alpha & \text { if } \mu_{1}=\mu_{2}
\end{array} \\
\lambda_{2} \doteq\left\{\begin{array}{cc}
\text { else } \\
\alpha+(1-\alpha) \exp \left(\frac{-c}{\left(\mu_{1}-\mu_{2}\right)^{2}}\right)
\end{array}\right.
\end{array}
$$

where $\mathrm{c}>0$. The small positive parameter $\alpha$ is mainly introduced for theoretical reasons. It keeps the diffusion tensor uniformly positive-definite.

Moving from the discrete domain to the continuous one for the purpose of completeness, we see that all of the previously mentioned diffusion filters can be cast in the form,

$$
\partial_{t} \mu=\operatorname{div}\left(D\left(J_{\rho}\right) \nabla \mu\right)
$$

Evidently, coherence enhancing diffusion uses the above equation, but also the diffusion tensor for edge enhancing diffusion can be written as,

$$
\begin{aligned}
& D=D\left(\nabla \mu_{\sigma} \nabla \mu_{\sigma}^{T}\right)=D\left(J_{0}\right) \nabla \mu_{\sigma} \\
& \text { while, the regularized isotropic nonlinear diffusion uses, } \\
& \mathrm{D}=\mathrm{D}\left(\left|\nabla \mu_{\sigma}\right|^{2}\right) I=D\left(\operatorname{trace}\left(\left(J_{0}\right) \nabla \mu_{\sigma}\right)\right) I
\end{aligned}
$$


and linear diffusion corresponds to $\mathbf{D}=\mathbf{I}$.

Let the diffusion tensor $\mathrm{D}$ be a $2 \times 2$ matrix. For the coherence enhancing anisotropic diffusion we need to perform a principal component analysis of the structure tensor J. Its eigenvalues are given by,

$$
\begin{aligned}
& \mu_{1}=\frac{1}{2}\left(J_{11}+J_{22}+\sqrt{\left(J_{11}-J_{22}\right)^{2}+4 J_{12}^{2}}\right) \\
& \mu_{2}=\frac{1}{2}\left(J_{11}+J_{22}-\sqrt{\left(J_{11}-J_{22}\right)^{2}+4 J_{12}^{2}}\right)
\end{aligned}
$$

and the first eigenvector $(\cos \psi, \sin \psi)^{T}$ satisfies,

$$
\left(\begin{array}{c}
\cos \psi \\
\sin \psi
\end{array}\right) \|\left(\begin{array}{c}
2 J_{12} \\
J_{22}-J_{11}+\sqrt{\left(J_{11}-J_{22}\right)^{2}+4 J_{12}^{2}}
\end{array}\right)
$$

The diffusion tensor uses identical eigenvectors and its eigenvalues are calculated from the eigenvalues of the structure tensor. An anisotropic diffusion filter can be written as,

$$
\begin{aligned}
& \partial_{t} u=\operatorname{div}(D \nabla u)=\operatorname{div}\left[\begin{array}{l}
A \partial_{x} u+B \partial_{y} u \\
B \partial_{x} u+C \partial_{y} u
\end{array}\right] \\
& =\partial_{x}\left(A \partial_{x} u\right)+\partial_{x}\left(B \partial_{y} u\right)+\partial_{y}\left(B \partial_{x} u\right)+\partial_{y}\left(C \partial_{y} u\right)
\end{aligned}
$$

Compared to isotropic nonlinear diffusion, the main novelty of this process is the mixed derivative expression $\partial_{x}\left(B \partial_{y} u\right)+\partial_{y}\left(B \partial_{x} u\right)$. The standard central difference approximations to these terms are,

$$
\begin{aligned}
& \approx \frac{1}{2 h_{1}}\left(B_{m+1, n} \frac{U_{m+1, n+1}-U_{m+1, n-1}}{2 h_{2}}-B_{m-1, n} \frac{U_{m-1, n+1}-U_{m-1, n-1}}{2 h_{2}}\right) \\
& \partial_{y}\left(B \partial_{x} u\right) \approx \frac{1}{2 h_{2}}\left(B_{m, n+1} \frac{U_{m+1, n+1}-U_{m-1, n+1}}{2 h_{1}}-B_{m, n-1} \frac{U_{m+1, n-1}-U_{m-1, n-1}}{2 h_{1}}\right)
\end{aligned}
$$

This scheme is quite stable even though it has undershoots and overshoots. This is also popular because of its simplicity. Thus we have seen a method which enforces the above given partial differential equation to enhance the image coherently. A detailed algorithm is presented in section 4. An example of the enhancement using this scheme is given below (figure 22). 

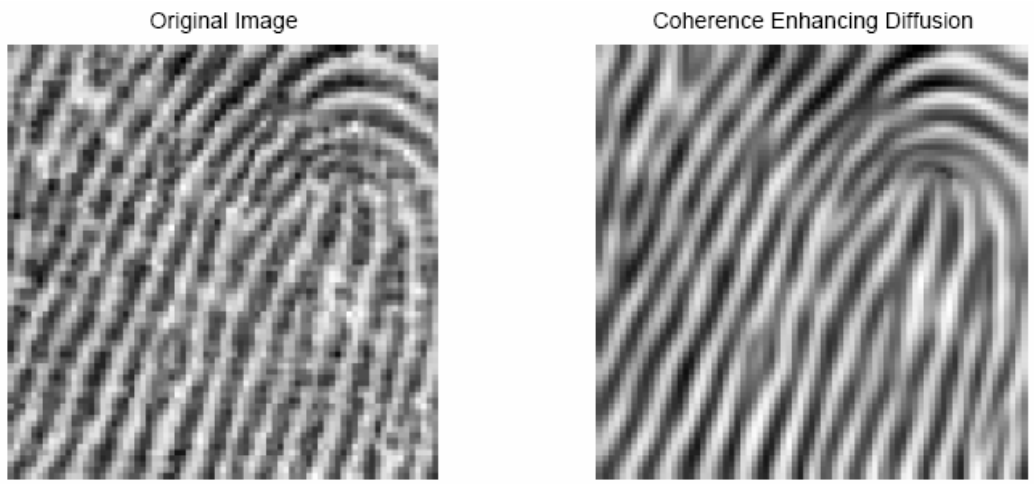

(1)
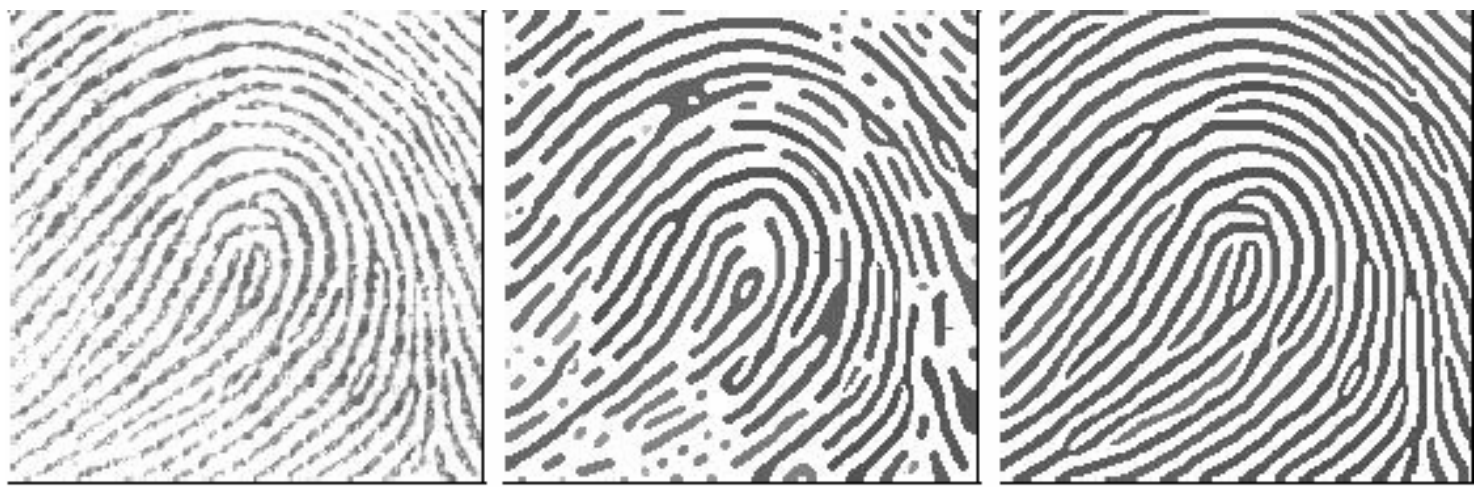

(2)

Figure 22: Anisotropic coherence enhancement of fingerprints

\subsection{Image Inpainting}

The modification of images in a way that is non detectable for an observer who does not know the original image is a practice as old as the Renaissance, the motives being often as much to bring medieval pictures up to date as to fill in any gaps. This process is coined Inpainting. The need to retouch the image in an unobtrusive way extended naturally from paintings to photography and film. The applications of this process vary from restoring or retouching old images and pictures or to restore damaged images either due to noise or due to transmission losses over the internet to the infamous "airbrushing" of political enemies. Digital techniques are starting to be widespread way of performing inpainting, ranging from attempts at fully automatic detection and removal of scratches in a film all the way to software tools that allow a sophisticated but mostly manual process [48]. 
It should be noted that classical image denoising algorithms do not apply for image inpainting. In common image enhancement applications, the pixels contain both information about the real data and the noise, whereas in image inpainting, there is no significant information in the region to be inpainted. The information is primarily in the regions surrounding the areas to be inpainted. The methods of inpainting can be broadly classified into three categories. The first deals with the restoration of films, the second one is related to texture analysis and the third one, a significantly less studied class is related to disocculsion.

Kokaram et al. [110] use motion estimation and autoregressive models to interpolate losses in films from adjacent frames. The basic idea is to copy into the gap the right pixels from neighboring frames. This technique is useless for still images or to films for which the regions to be inpainted span many frames.

Hirani and Totsuka [111] combine frequency and spatial domain information in order to fill a given region with a selected texture. This is a very simple technique that produces incredibly good results. On the other hand the algorithm deals only with texture synthesis and not with structured background, and requires the user to select the texture to be copied into the region to be inpainted. Other texture synthesis algorithms [112], [113], [114] can be used as well to recreate a pre-selected texture to fill in a region to be inpainted.

In the group of disocclusion algorithms, a pioneering work is described in [115]; Nitzberg et al. presented a technique for removing occlusions with a goal of image segmentation. Because the region to be inpainted can be considered as occluding objects, removing occlusions is analogous to image inpainting. The basic idea is to connect T-junctions at the same gray level with elastica-minimizing curves. This technique was mainly developed for simple images, with only a few objects with constant gray levels, and will not be applicable for the examples with natural images. Masnou and Morel [116] extended these ideas, presenting a very inspired general variational formulation for 
disocculsion and a practical algorithm, which performs inpainting by joining with geodesic curves the points of the isohyets arriving at the boundary of the region to be inpainted. The regions to be inpainted are limited to having simple topology. In addition, the angle with which the level lines arrive at the boundary of the inpainted region is not well preserved.

If $\Omega$ is the region to be inpainted, and $\partial \Omega$ its boundary, the technique proposed will prolong the isophyte lines arriving at $\partial \Omega$, while maintaining the angle of arrival. The aim is to proceed drawing from $\partial \Omega$ inward in this way, while curving the prolongation lines progressively to prevent them from crossing each other.

Before turning to the mathematical derivation of the formula, let us look into the traditional theory of inpainting and analyze it. Inpainting is a very subjective procedure, different for each work of art and different profession. Though the technique of solving the problem is not unique, the common methodology is as follows,

1. The global picture determines how to fill in the gap, the purpose of inpainting being to restore the unity of work.

2. The structure of the area surrounding $\Omega$ is continued into the gap, contour lines are drawn by means of the prolongation of those arriving at $\partial \Omega$.

3. The different regions inside $\Omega$, as defined by the contour lines, are filled with color, matching those of $\partial \Omega$.

4. The small details are painted, like small holes etc. This effectively means addition of texture information in the image. 

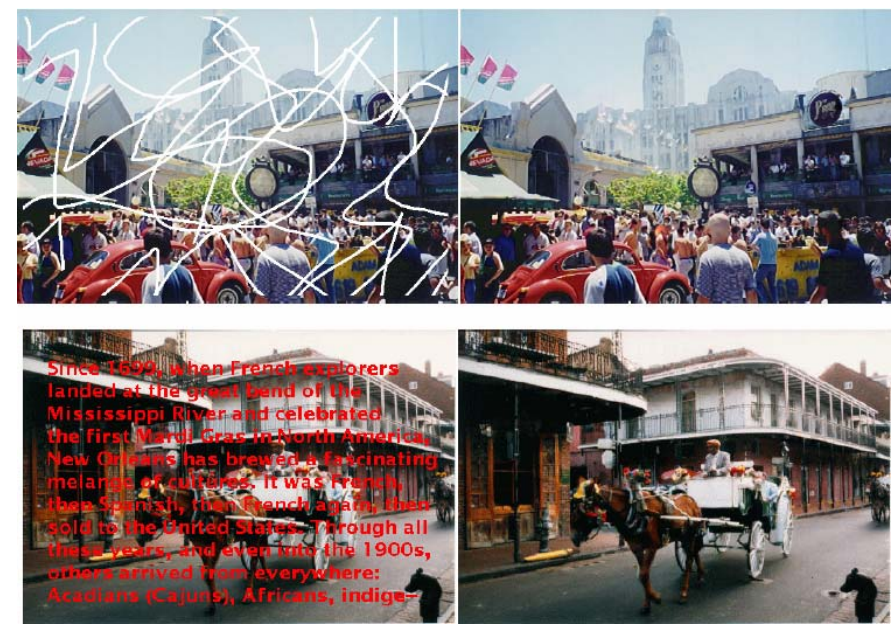

Figure 23: Inpainting applications and examples [48]

The algorithm can be constructed effectively by repeating steps 2 and 3. Thus the gap $\Omega$ can be shrunk by prolonging inward, in a smooth way, the lines arriving at the gap boundary $\partial \Omega$. Let us now look how to translate the analytical thoughts into the mathematical domain which will give us a working inpainting algorithm in the end.

Let,

$$
I_{0}(i, j):[0, M] x[0, N] \rightarrow \mathbb{R}, \text { with }[0, M] x[0, N] \subset \mathbb{N} x \mathbb{N}
$$

be a discrete 2D gray-level image. From the description of the manual inpainting techniques, an iterative algorithm seems a natural choice. The digital inpainting procedure will construct a family of images,

$$
\begin{aligned}
& I(i, j, n):[0, M] x[0, N] x \mathbb{N} \rightarrow \mathbb{R}, \\
& \text { such that } I(i, j, 0)=I_{0}(i, j) \\
& \text { and } \lim _{n \rightarrow \infty} I(i, j, n)=I_{R}(i, j), \\
& \text { where, } I_{R}(i, j) \text { is the output of the algorithm }
\end{aligned}
$$

Any general algorithm of that form can be written as,

$$
I^{n+1}(i, j)=I^{n}(i, j)+\Delta t I_{t}^{n}(i, j), \forall(i, j) \in \Omega
$$

Where,

The superscript ' $n$ ' denotes the inpainting time $n$, 
$(i, j)$ are the pixel coordinates,

$\Delta t$ is the rate of improvement, and

$I_{t}^{n}(i, j)$ stands for the update of the image.

One important thing to observe is that this evolution equation acts only inside the inpainting domain or the region to be inpainted.

Thus with this equation, the image $I^{n+1}(i, j)$ is an improved version of $I^{n}(i, j)$, with the improvement given by $I_{t}^{n}(i, j)$. As $n$ increases, we achieve a better image. Now we need to design the equation for the update $I_{t}^{n}(i, j)$.

As suggested by our analysis of the manual inpainting technique, we need to continue the lines arriving at the boundary $\partial \Omega$ of the region $\Omega$ to be inpainted. We need to propagate smoothly the information from outside $\Omega$ into $\Omega$. Let $\mathrm{L}^{\mathrm{n}}(\mathrm{i}, \mathrm{j})$ be the information that we want to propagate and $\vec{N}^{n}(i, j)$ the propagation direction, this means that we must have,

$$
I_{t}^{n}(i, j)=\delta \vec{L}^{n}(i, j) \cdot \vec{N}^{n}(i, j)
$$

Where, $\delta \vec{L}^{n}(i, j)$ is a measure of the change in the information $\mathrm{L}^{\mathrm{n}}(\mathrm{i}, \mathrm{j})$. With this equation, we estimate the information $\mathrm{L}^{\mathrm{n}}(\mathrm{i}, \mathrm{j})$ of our image and compute its change along the $\vec{N}$ direction. At steady state or when the algorithm converges, $I^{n+1}(i, j)=I^{n}(i, j)$ and from the above equations we would have $\delta \vec{L}^{n}(i, j) \cdot \vec{N}^{n}(i, j)=0$, which implies that the information $\mathrm{L}$ has propagated exactly in the right direction $\mathrm{N}$. 


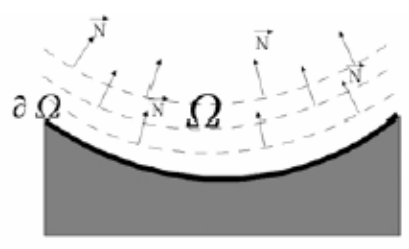

Figure 1: Propagation direction as the normal to the signed distance to the boundary of the region to be inpainted.

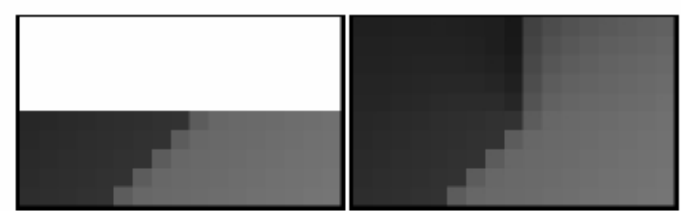

Figure 2: Unsuccessful choice of the information propagation direction. Left: detail of the original image, region to be inpainted is in white. Right: restoration.

Figure 24: Right choice of inpainting direction [48]

Now all that we have to do is to find out the right representation of $\mathrm{L}$ and the direction of propagation $\mathrm{N}$. As we want the propagation to be smooth, $\mathrm{L}^{\mathrm{n}}(\mathrm{i}, \mathrm{j})$ should be an image smoothness estimator. For this purpose, we may use a simple discrete implementation of the Laplacian:

$$
L^{n}(i, j):=I_{x x}^{n}(i, j)+I_{y y}^{n}(i, j)
$$

For computing the change $\delta \vec{L}^{n}(i, j)$ of this value along $\vec{N}$, we must define what the direction $\vec{N}$ for the 2D information propagation will be. One possibility is to define $\vec{N}$ as the normal to the signed distance to $\partial \Omega$. This choice is motivated by the belief that propagation normal to the boundary will lead to the continuity of the isophytes at the boundary. But an unforeseen blunder is that the lines arriving at $\partial \Omega$ curve in order to align with $N$. If the isophytes tend to align with $N$ the best choice for $N$ is then the isophytes direction.

To solve this problem, a time varying estimation of the isophytes direction field is found out. For any given point $(i, j)$, the discretized gradient vector $\nabla I^{n}(i, j)$ gives the direction of largest spatial change, and its 90 degrees rotation $\nabla^{\perp} I^{n}(i, j)$ is the direction of smallest spatial change, so the vector $\nabla^{\perp} I^{n}(i, j)$ gives the isophytes direction. The field $N$ 
is then given by the time varying $\vec{N}(i, j, n)=\nabla^{\perp} I^{n}(i, j)$. Thus we use a time varying estimation that is coarse at the beginning but progressively achieves the desired continuity at $\partial \Omega$, instead of a fixed field $N$ that would imply knowledge of the directions of the isophytes from the start.

It can be easily observed that the direction field is not normalized. The reason for this choice relies on the numerical stability of the algorithm, which will be discussed further. As the inpainting is done along the isophytes, it is irrelevant if $\nabla^{\perp} I^{n}(i, j)$ is obtained as a clockwise or counterclockwise rotation of $\nabla I^{n}(i, j)$. In both the cases, the change of $\mathrm{I}^{\mathrm{n}}(\mathrm{i}$, j) along those directions should be minimum. Thus in short, we are estimating a variation of the smoothness, given by a discretization of the 2D Laplacian and then project this variation into the isophytes direction. This projection is used to update the value of the image inside the region to be inpainted.

To ensure a correct evolution of the directional field, a diffusion process is interleaved with the image inpainting process described above. So for every few steps we apply a few iterations of image diffusion. This diffusion corresponds to the periodical curving of lines to avoid them from crossing each other. Anisotropic diffusion is used in order to achieve this goal without losing sharpness in the reconstruction. The following equation is used:

$$
\frac{\partial I}{\partial t}(x, y, t)=g_{\in}(x, y) \kappa(x, y, t)|\nabla I(x, y, t)|, \forall(x, y) \in \Omega^{\epsilon}
$$

Where $\Omega^{\epsilon}$ is a dilation of $\Omega$ with a ball of radius $\in, \kappa$ is the Euclidean curvature of the isophytes of we, and $g_{\in}(x, y)$ is a smooth function in $\Omega^{\epsilon}$ such that $g_{\in}(x, y)=0$ in $\Omega^{\epsilon} / \Omega$ and $g_{\epsilon}(x, y)=1$ at the set of points of $\Omega$ whose distance $\partial \Omega$ is larger than $\in$. 
Super Resolution

\section{Chapter 4}

\section{Super Resolution}

In video sequences there is usually a large overlap between successive frames; a region in the scene is sampled in several different images due to the camera motion. This super sampling information can sometime be used to achieve an image of the region with higher spatial resolution. The process of reconstructing a high resolution image from several different images covering the same region is called Static Super Resolution.

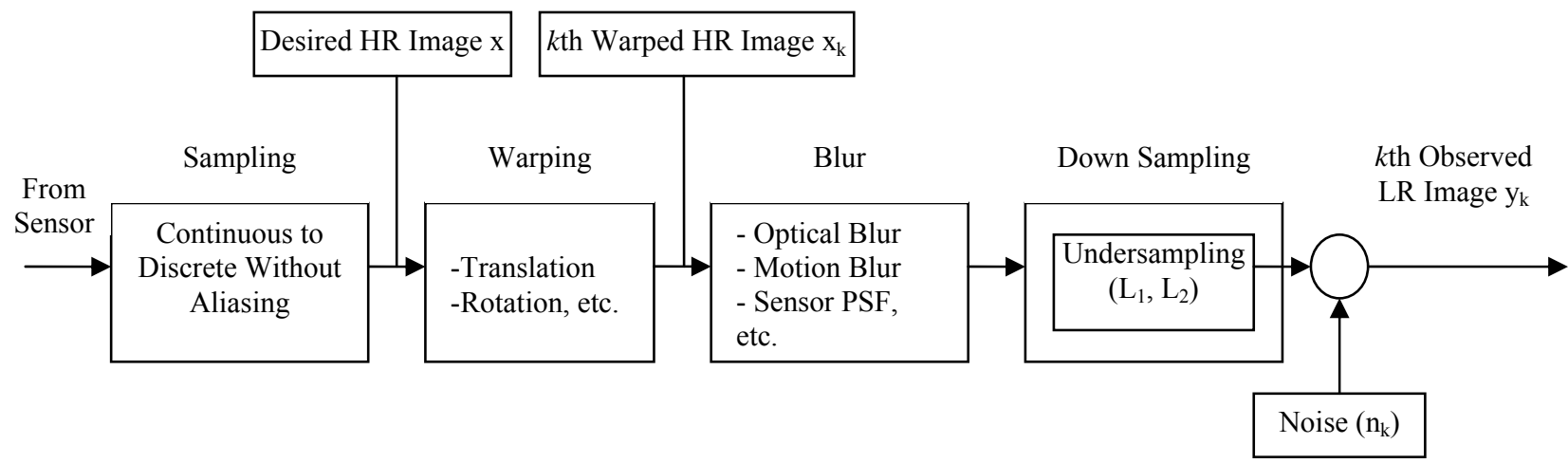

Figure 25: Relation between a low resolution and a high resolution image w.r.t noise
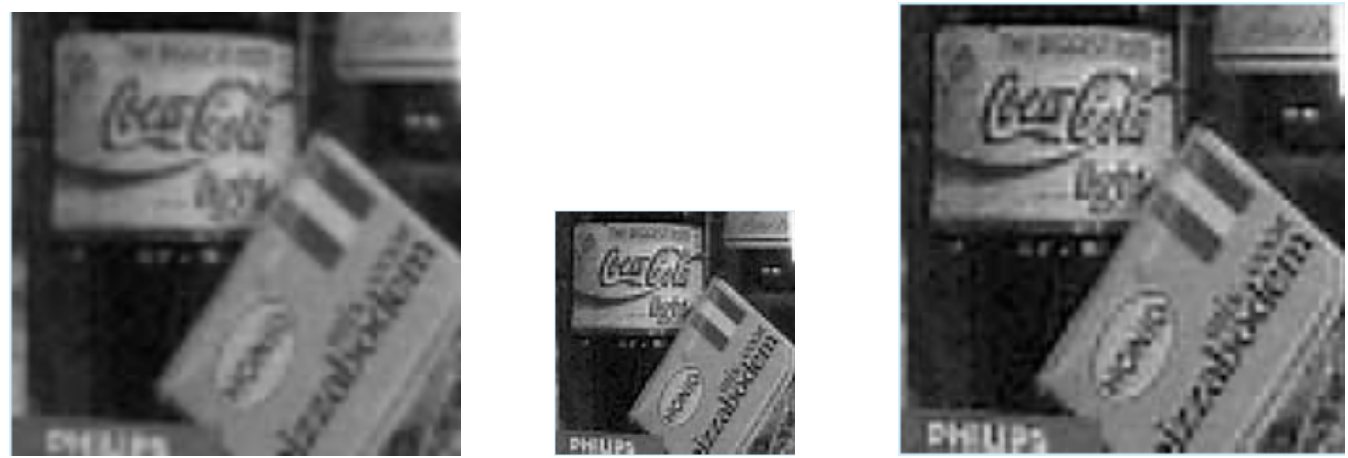

Figure 26: Image interpolated by bilinear interpolation and by a super resolution technique

A similar problem is Dynamic Super Resolution, in which the output of the algorithm is a sequence of high resolution images, instead of a single image. In this work we are 
focusing on the static case, although the ideas presented here can be easily generalized to the dynamic case. The super-resolution reconstruction problem is an inverse problem, dealing with the recovery of a single high-resolution image from a set of low quality images. In its general form, the super resolution problem may consist of images with arbitrary geometric warp, space variant blur and colored noise.

Compressed video is rapidly becoming the preferred method for video delivery. Applications such as Internet streaming, wireless videophones, DVD players and HDTV devices all rely on compression techniques and each requires a significant amount of data reduction for commercial viability. To introduce this reduction, a specific application often employs a low-resolution sensor or sub-samples the original image sequence. The reduced resolution sequence is then compressed in a lossy manner, which produces an estimate of the low-resolution data.

For many tasks, the initial reconstruction of the compressed sequence is acceptable for viewing. However, when an application requires a high-resolution frame or image sequence, a super-resolution algorithm must be employed. Super-resolution algorithms recover information about the original high resolution image by exploiting sub-pixel shifts in the low-resolution data. These shifts are introduced by motion in the sequence and make it possible to observe samples from the high-resolution image that may not appear in a single low-resolution frame.

As stated earlier, a super resolution problem can be divided into three main subcategories,

\section{Image Alignment}

2. Image Interpolation

3. Image Restoration/Enhancement.

In chapters 2 and 3 we saw different methods of performing the same and the methods which we have chosen and implemented to do them. Now in this section we will see how 
we can combine these algorithms to give a final super resolution algorithm. Let us formally define the super resolution process by giving the flowchart of the whole process,

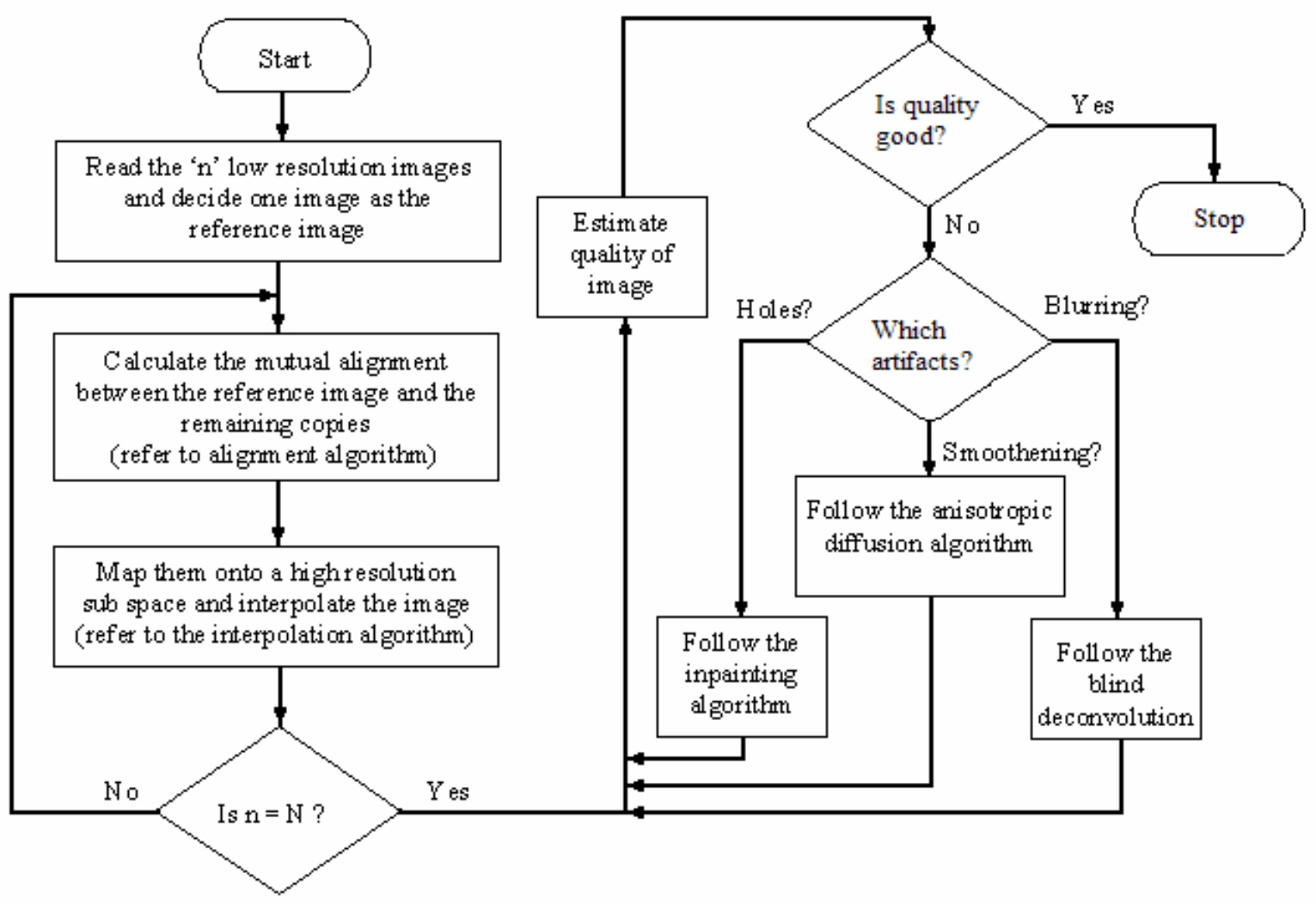

Figure 27: Proposed super resolution process

\subsection{Image Alignment}

For image alignment we have chosen to use the phase correlation method in both the frequency domain and the log polar domain to estimate the affine transformation parameters of rotation and translation. The image is converted into the frequency domain using standard fast fourier transforms and the correlation between the different images give the translational parameters. Now for the estimation of the rotational parameters, the original images are converted into the log polar domain as shown earlier and these images are then converted into the frequency domain for the correlation to give the rotational parameters. 
This changes the images considerably and thus the translational parameters are again estimated. Thus this process of estimating the translational parameters first and then going for the rotational parameters is iteratively done, until the two images do not differ by more than 1 pixel width in both the $\mathrm{X}$ and the $\mathrm{Y}$ direction and the angular difference between them is zero. As specified in the theory in section 2, the alignment is obtained in the sub pixel domain and thus finally we have three parameters as an estimation of the affine parameters for the high resolution grid.

An algorithm for finding the affine parameters using the phase correlation domain using the log polar and the frequency domain is given below.

1. Let image 0 and image 1 be the two images whose affine parameters are to be estimated. We define image 0 as the reference image and image 1 as the acquired image.

2. While the affine parameters theta, $x_{-}$estimate and $y_{-}$estimate greater than the defined threshold,

a. Convert images image 0 and image 1 into its frequency domain representation using Fast Fourier Transforms.

b. Calculate the $x_{-}$estimate' and y_estimate' using the phase correlation method described earlier in the sub pixel domain.

c. Translate image1 by (-x_estimate',-y_estimate') and modify the final_x and final_y correspondingly.

d. Convert the images image 0 and image1_translate into the log polar domain using 'bicubic' interpolation into logpolar0 and logpolar1.

e. Now convert these $2 \log$ polar images into the frequency domain using fast fourier transforms.

f. Calculate the theta_estimate as the $\mathrm{x}$ coordinate of the peak using the phase correlation method.

g. Rotate image1_translate by angle - theta and modify the final_theta correspondingly.

h. Repeat the above process till we achieve the predefined threshold.

3. The final affine parameters are given by final_x,final_y and final_theta. 
Super Resolution

\subsection{Image Interpolation}

After this process of image alignment or registration, the next step is the interpolation part. In this, initially the two or more low resolution images are mapped onto the high resolution grid with the estimated affine parameters and then a POCS based method interpolates the images to give the required high resolution image. The general algorithm can be given as follows. Please also follow the equations for the constraints given in the theory.

1. Start with all the available low resolution images. Interpolate them onto the desired high resolution sub space. This being an initial guess can be any method, like bicubic, bilinear etc. we have chosen bilinear as the initial guess.

2. Now the affine parameter calculation function is executed between the required image or the base copy and the remaining low resolution images.

3. These parameters are then evaluated into the high resolution subspace and depending on their values; they are mapped onto the grid. Now it should be noted here that the affine parameters give a continuous subspace and that has to be again approximated to the nearest integer values with the help of certain interpolation methods.

4. Now with the help of the constraints given in the theory above, use the classical theory of alternating projections onto the convex sets to come up with an estimate of the high resolution image.

5. Given in the POCS model, is assumption of a normalized linear-shift-invariant (LSI) Point Spread Function (PSF). This PSF has a certain effective range. This PSF is overlaid on the transformed position, and the current values of all the high resolution grid points within the window are multiplied by the PSF value at that grid point. We have used a Gaussian PSF for this purpose. Given these new values, we can infer an expected value at the position. The step described in this process can be generally thought of as using the transformed low resolution pixel location to scale the high resolution pixel, and then use those values to infer the original value stored in the transformed pixel. 
6. This value is a result only of the PSF being combined with the high resolution values, and has nothing yet to do with the value from the low resolution grid. Now the residual between the two is defined as a simple difference between this PSF value and the constraint imposed value. This residual is multiplied by the PSF and placed on the corresponding point on the high resolution grid.

7. The above process is conducted for all the low resolution images until we get a good estimate of the high resolution image

Now the image thus obtained after the alignment and the interpolation step is a little bit blurred or has holes or may be noisy depending on the quality of the images and the loss of obtaining the high frequency components after the interpolation step. Thus depending on the type of image reconstructed, the next step is to enhance this image to obtain the required high resolution image. We have proposed to use three image enhancement techniques for tackling the above problems. We have discussed them in the $3^{\text {rd }}$ section and here we present a method to combine each of these individual processes to give the required high resolution image.

For the enhancement process, we have chosen to use the following three methods,

1. Blind Deconvolution

2. Anisotropic Coherence Enhancement

3. Image Inpainting

\subsection{Blind Deconvolution}

The first method is the blind deconvolution method which assumes nothing about the blurring kernel and starting from an arbitrary kernel, it tries to come up with inverse kernels which will deblurr the image in the best possible format. The concept of conjugate directions has been introduced successfully in optimization theory, and was motivated by the need to accelerate the steepest descent methods. One of the advantages of this method is that convergence in a finite number of iterations is guaranteed when a quadratic cost function is used and exact arithmetic is assumed. Even for nonquadratic 
costs, the method shows considerably increased convergence speed. The algorithm is based on the premise that information about the curvature of $\mathrm{J}$ with each new iteration can accelerate convergence. The "best" direction to move is not always the instantaneously steepest direction.

The algorithm for this method can be summarized as:

- $\quad$ Set initial conditions $(\mathrm{k}=0)$ :

$$
\begin{aligned}
u_{k}^{T} & =\left[u_{k}(1,1), \ldots, u_{k}\left(\left(N_{x u}+1\right) / 2,\left(N_{y u}+1\right) / 2\right), \ldots, u_{k}\left(N_{x u}, N_{y u}\right)\right] \\
& =[0, \ldots, 1, \ldots, 0] \\
\delta & >0 \text { is set. }
\end{aligned}
$$

- At iteration $(\mathrm{k}): \mathrm{k}=0,1,2, \ldots$

1. If $J\left(u_{k}\right) \leq \delta$, stop

2. If $\mathrm{k}=0, d_{k}=-\nabla J\left(u_{k}\right)$.Otherwise, $d_{k}=-\nabla J\left(u_{k}\right)+\beta_{k-1} d_{k-1}$

3. Perform a line minimization to find $t_{k}$ such that,

$$
J\left(u_{k}+t_{k} d_{k}\right) \leq J\left(u_{k}+t d_{k}\right) \text { for } t \in \mathbb{R}
$$

4. $u_{k+1}=u_{k}+t d_{k}$

Where, $u_{k} \in \mathbb{R}^{N_{x u} N_{y u}}$ is the current estimate of the inverse filter, and

$$
\begin{aligned}
& {\left[\nabla J\left(u_{k}\right)\right]_{j+(i-1) N_{x u}, 1}=\frac{\partial J\left(u_{k}\right)}{\partial u(i, j)}} \\
& =2 \sum_{(x, y) \in D_{\text {neg }}} \hat{f}_{k}(x, y) g(x-i+1, y-j+1) \\
& +2 \sum_{(x, y) \in D_{\text {sup }}}\left[\hat{f}_{k}(x, y)-L_{B}\right] g(x-i+1, y-j+1) \\
& \text { where, } \hat{f}_{k}(x, y)=u_{k}(x, y) * g(x, y)
\end{aligned}
$$

And, $\beta_{k}=\frac{\left\langle\left|\nabla J\left(u_{k}+1\right)-\nabla J\left(u_{k}\right), \nabla J\left(u_{k}+1\right)\right|\right\rangle}{\left\|\nabla J\left(u_{k}\right)\right\|^{2}}$

\subsection{Anisotropic Coherence Enhancement}

The second method is the anisotropic diffusion based coherence enhancement. In these situations estimating the local orientation as the direction of the gradient vector is not possible. In this application, image smoothing is required, where we do like to smooth the 
image, without destroying the finger print lines. The isotropic Gaussian smoothing is of no great help here as it will destroy the ridge pattern. We want to find the prominent (perceptual) local orientation and smooth along the finger print lines but not across them. The local orientation estimation is based on the structure tensor:

$$
S=\left(\begin{array}{ll}
S_{11} & S_{12} \\
S_{12} & S_{22}
\end{array}\right)=\left(\begin{array}{ll}
L_{x} L_{x} * G^{u} & L_{x} L_{y} * G^{u} \\
L_{x} L_{y} * G^{u} & L_{y} L_{y} * G^{u}
\end{array}\right)
$$

Whose eigenvectors indicate the most prominent orientation. The difference between the two eigenvalues is an indication of the anisotropy in a local neighborhood in the image. Coherence enhancing diffusion constructs the diffusion tensor D as follows:

$$
D=\left(\begin{array}{ll}
d_{11} & d_{12} \\
d_{12} & d_{22}
\end{array}\right)=R^{T}\left(\begin{array}{cc}
c_{1} & 0 \\
0 & c_{2}
\end{array}\right) R
$$

Here is the rotation matrix whose columns are the eigenvectors of the structure tensor $\mathrm{S}$ (i.e. indicting the local orientation of an image patch) and c1 and c2 are the conductivity coefficients along the principal directions. The eigenvectors of the structure tensor and thus the rotation matrix $\mathrm{R}$ can be calculated analytically. This leads to the diffusion tensor D with components:

$$
\begin{aligned}
& d_{11}=\frac{1}{2}\left(c_{1}+c_{2}+\frac{\left(c_{2}-c_{1}\right)\left(s_{11}-s_{22}\right)}{\alpha}\right) \\
& d_{12}=\frac{\left(c_{2}-c_{1}\right) s_{12}}{\alpha} \\
& d_{22}=\frac{1}{2}\left(c_{1}+c_{2}-\frac{\left(c_{2}-c_{1}\right)\left(s_{11}-s_{22}\right)}{\alpha}\right) \\
& \text { where, } \alpha=\sqrt{\left(s_{11}-s_{22}\right)^{2}+4 s_{12}^{2}}
\end{aligned}
$$

The eigenvalues of the structure tensor are given by,

$$
\lambda_{1,2}=\frac{1}{2}\left(s_{11}+s_{22} \pm \alpha\right)
$$

These eigenvalues define the diffusion 'speeds' c1 \& c2 as,

$$
c_{1}=\max \left(0.01,1-e^{-\left(\lambda_{1}-\lambda_{2}\right)^{2} / k^{2}}\right) ; c_{2}=0.01
$$


This completes the calculation and formulae of the coherence enhancement parameters. Its algorithm can be stated as;

1. Calculate the Gaussian derivatives of the image in both $\mathrm{X}$ and $\mathrm{Y}$ directions. This gives the $\mathrm{Lx}$ and $\mathrm{Ly}$ as specified in the above formulas.

2. Calculate the structure tensor S given by the above formula using the Gaussian derivatives, S11, S12, S21 \& S22.

3. Calculate the value of alpha by the above formula.

4. Calculate the eigen values of the structure tensor (Lambda) given the formulae.

5. Similarly calculate the conductivity coefficients $\mathrm{c} 1$ and $\mathrm{c} 2$.

6. Calculate the diffusion tensor and the rotation matrix by the stated formulae.

7. Approximate the discrete implementation scheme $\mathrm{dL} / \mathrm{dt}$ to calculate the new estimate given the original estimate and the diffusion tensor with a specified step size.

8. Repeat the above steps until the error between the previous estimate and the current estimate is above the given threshold.

\subsection{Image Inpainting}

The third and the final restoration scheme used is the image inpainting method. In this the only inputs to the system are the image to be inpainted and the mask that delimits the portion to be inpainted. As stated earlier, the whole image undergoes anisotropic diffusion smoothing as a preprocessing step. This minimizes the influence of noise on the estimation of the direction of the isophytes. Now the inpainting process starts where only the image defined by the inpainting domain is modified. Again as stated a step of anisotropic diffusion is applied until a steady state is achieved.

The discrete inpainting algorithm equations are as follows:

1. Let $I^{n}(i, j)$ stand for each one of the image pixels inside the region $\Omega$ at the inpainting time $\mathrm{n}$. Then the discrete inpainting equation borrowed from the numerical analysis literature is given by, 
$I^{n+1}(i, j)=I^{n}(i, j)+\Delta t I_{t}^{n}(i, j), \forall(i, j) \in \Omega$

where,

$I_{t}^{n}(i, j)=\left[\delta \vec{L}^{n}(i, j) \cdot \frac{\vec{N}(i, j, n)}{|\vec{N}(i, j, n)|}\right]\left|\nabla I^{n}(i, j)\right|$

2. The measure of the change of information, which can also be called the smoothness measure, is given by,

$\delta \vec{L}^{n}(i, j):=\left[L^{n}(i+1, j)-L^{n}(i-1, j), L^{n}(i, j+1)-L^{n}(i, j-1)\right]$,

$L^{n}(i, j)=I_{x x}^{n}(i, j)+I_{y y}^{n}(i, j)$,

3. Calculate the isophyte direction by,

$$
\begin{aligned}
& \frac{\vec{N}(i, j, n)}{|\vec{N}(i, j, n)|}:=\frac{\left[-I_{x}^{n}(i, j), I_{y}^{n}(i, j)\right]}{\sqrt{\left[I_{x}^{n}(i, j)\right]^{2}+\left[I_{y}^{n}(i, j)\right]^{2}}}, \\
& \beta^{n}(i, j)=\delta \vec{L}^{n}(i, j) \cdot \frac{\vec{N}(i, j, n)}{|\vec{N}(i, j, n)|},
\end{aligned}
$$

4. Then we calculate $\beta^{n}$, which is the projection of $\delta \vec{L}^{n}$ onto the normalized vector $N$, by the above equation. Then we multiply this projection with a slope limited version of the norm of the gradient of the image. A central difference realization would turn the scheme unstable and thus we use the slope limiters.

5. The final modification parameter is given by the following equation.

$\left|\nabla I^{n}(i, j)\right|= \begin{cases}\sqrt{\left(I_{x b m}^{n}\right)^{2}+\left(I_{x f M}^{n}\right)^{2}+\left(I_{y b m}^{n}\right)^{2}+\left(I_{y f M}^{n}\right)^{2}} & \text { when } \beta^{n}>0 \\ \sqrt{\left(I_{x b M}^{n}\right)^{2}+\left(I_{x f m}^{n}\right)^{2}+\left(I_{y b M}^{n}\right)^{2}+\left(I_{y f m}^{n}\right)^{2}} & \text { when } \beta^{n}<0\end{cases}$

The subscripts $\mathrm{b}$ and $\mathrm{f}$ denote backward and forward differences respectively and the subscripts $\mathrm{m}$ and $\mathrm{M}$ denote the minimum and the maximum respectively, between the derivative and zero.

6. The above process is repeated until we fill in all the holes given by the estimate mask. 


\section{Chapter 5}

\section{Results}

As the whole motive for the development of the algorithm is to improve or enhance the resolution of biometric images, we believe that the results can be more appreciated by the visual inspection of the enhanced images. We will be supplying with mathematical values of the error but the reader is advised to note the focus is on the improvement in the quality of the image.

Before getting onto the results for the super resolution process, let us appreciate the results for the individual modules which make up the whole resolution enhancement process. As described earlier, this thesis has 3 main parts, the alignment, the interpolation and the restoration. The restoration in turn has again three parts as blind deconvolution, anisotropic coherence enhancement and inpainting. Let us look at the results for these individual modules one by one.

\subsection{Image Alignment}

To simulate the conditions for this experiment, we have come up with the following setup. We consider a biometric image (iris and fingerprint) and apply some affine transformations to it. Thus now we have two images with some affine transformation between them.

Now we down sample these copies by a certain factor ( 2 in this case) and pick up any copy out of the 4 copies created. This is due to the fact that finally during the super resolution process, one will not have any knowledge of which copy is presented to him.

Now these two images undergo the image alignment process and we have the estimated parameters of translation and rotation as the output. These parameters are compared with the original theoretical parameters to give the accuracy of this algorithm. 
1. Iris Image:
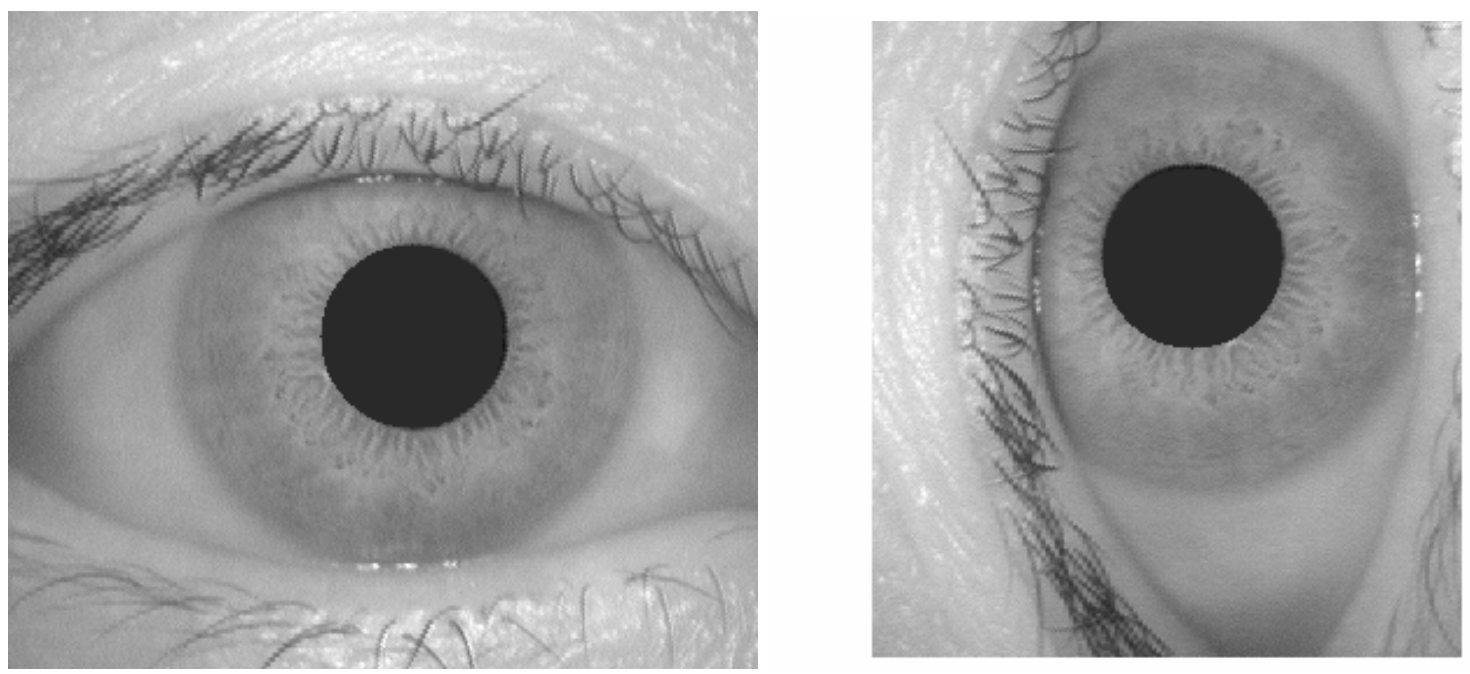

(a) Original down sampled copy

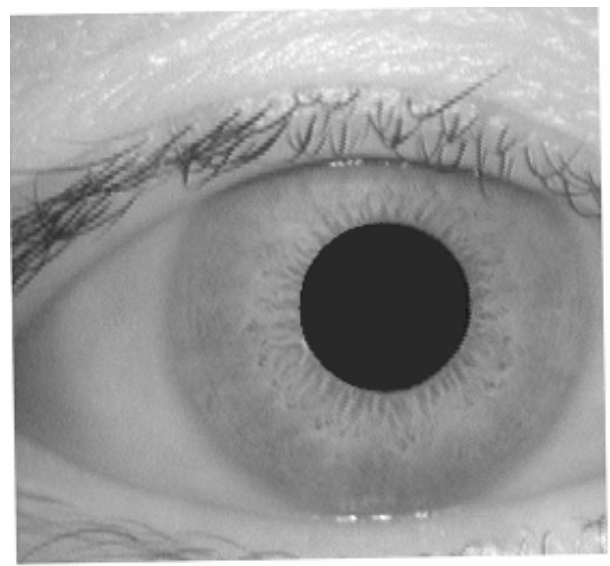

(b) down sampled copy with affine transformation

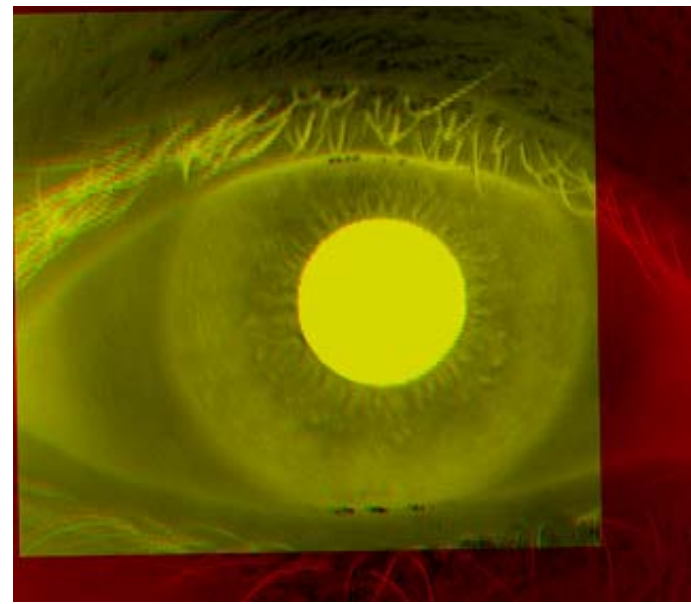

(c) Transformed version of (b) after execution (d) visualization of the alignment

Figure 28: Iris image alignment using phase correlation

\begin{tabular}{|c|c|c|c|}
\hline & Rotational Parameters & \multicolumn{2}{|c|}{ Translational Parameters } \\
\cline { 3 - 4 } & 90 & X direction & Y direction \\
\hline $\begin{array}{c}\text { Original Resolution } \\
\text { (Theoretical Values) }\end{array}$ & 90 & 26.5 & 51 \\
\hline $\begin{array}{c}\text { Down sampled values } \\
\text { (theoretical) }\end{array}$ & 89 & 26.71 & 25.5 \\
\hline $\begin{array}{c}\text { Down sampled estimated values } \\
\text { (observed) }\end{array}$ & 89 & 53.42 & 52.64 \\
\hline Observed values & 90.32 \\
\hline
\end{tabular}

Table 1: Comparison of theoretical and achieved affine parameters for iris image 
2. Fingerprint Image

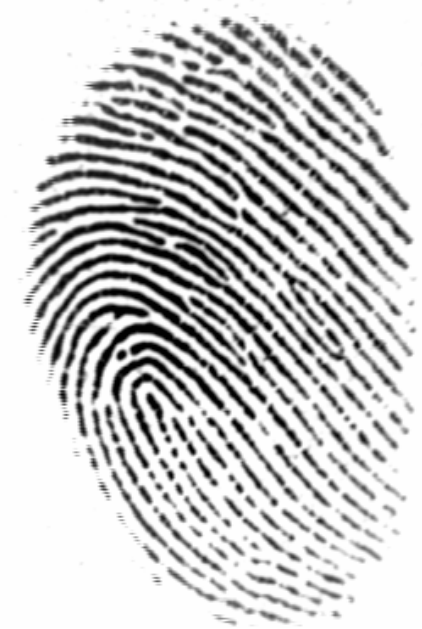

(a) Original down sampled copy

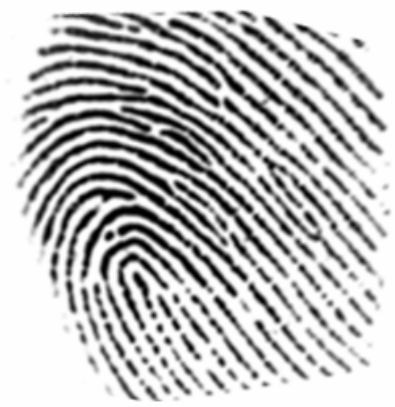

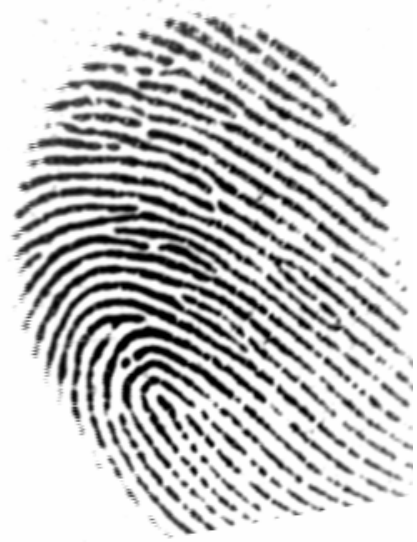

(b) down sampled copy with affine transformation

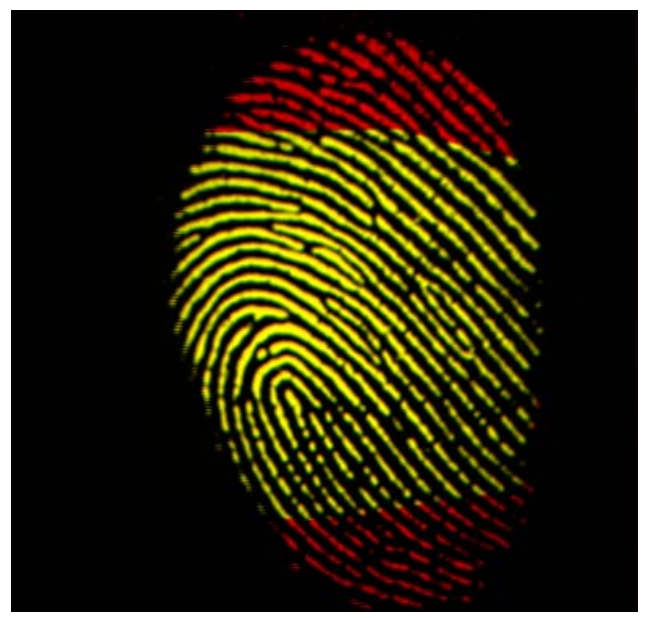

(c) Transformed version of (b) after execution (d) visualization of the alignment

Figure 29: Fingerprint image alignment using phase correlation

\begin{tabular}{|c|c|c|c|}
\hline & Rotational Parameters & \multicolumn{2}{|c|}{ Translational Parameters } \\
\cline { 3 - 4 } & 10 & X direction & Y direction \\
\hline $\begin{array}{c}\text { Original Resolution (Theoretical } \\
\text { Values) }\end{array}$ & 10 & 53.5 & 95 \\
\hline $\begin{array}{c}\text { Down sampled values } \\
\text { (theoretical) }\end{array}$ & 10 & 52.94 & 47.45 \\
\hline $\begin{array}{c}\text { Down sampled estimated values } \\
\text { (observed) }\end{array}$ & 10 & 105.88 & 94.9 \\
\hline Observed values & 10 & \\
\hline
\end{tabular}

Table 2: Comparison of theoretical and achieved affine parameters for fingerprint image 


\subsection{Image Interpolation}

In the following sub section we present the results for the interpolation algorithm. As stated earlier, we are following the projection on to the convex sets (POCS) method. We present first the two images from which the final image was created. One of these images is the reference image and the others are the copies of the same fingerprint. It may be recalled that this interpolated image is then given as input to the image restoration algorithm which will enhance the raw image to give the final image.

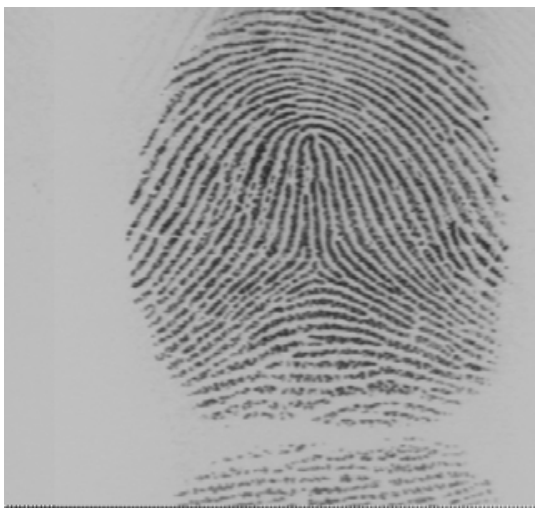

(a) Fingerprint copy used for interpolation

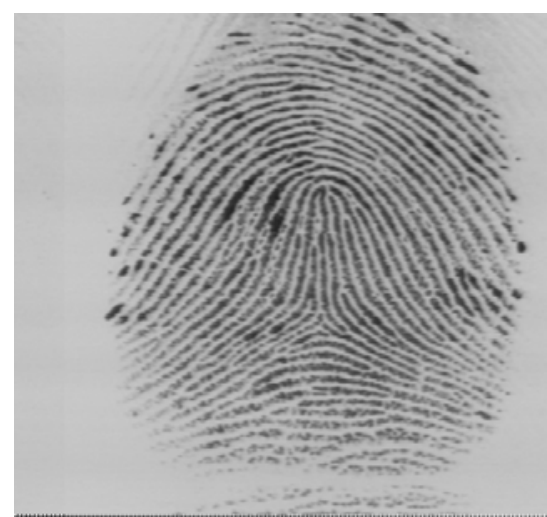

(b) Reference copy

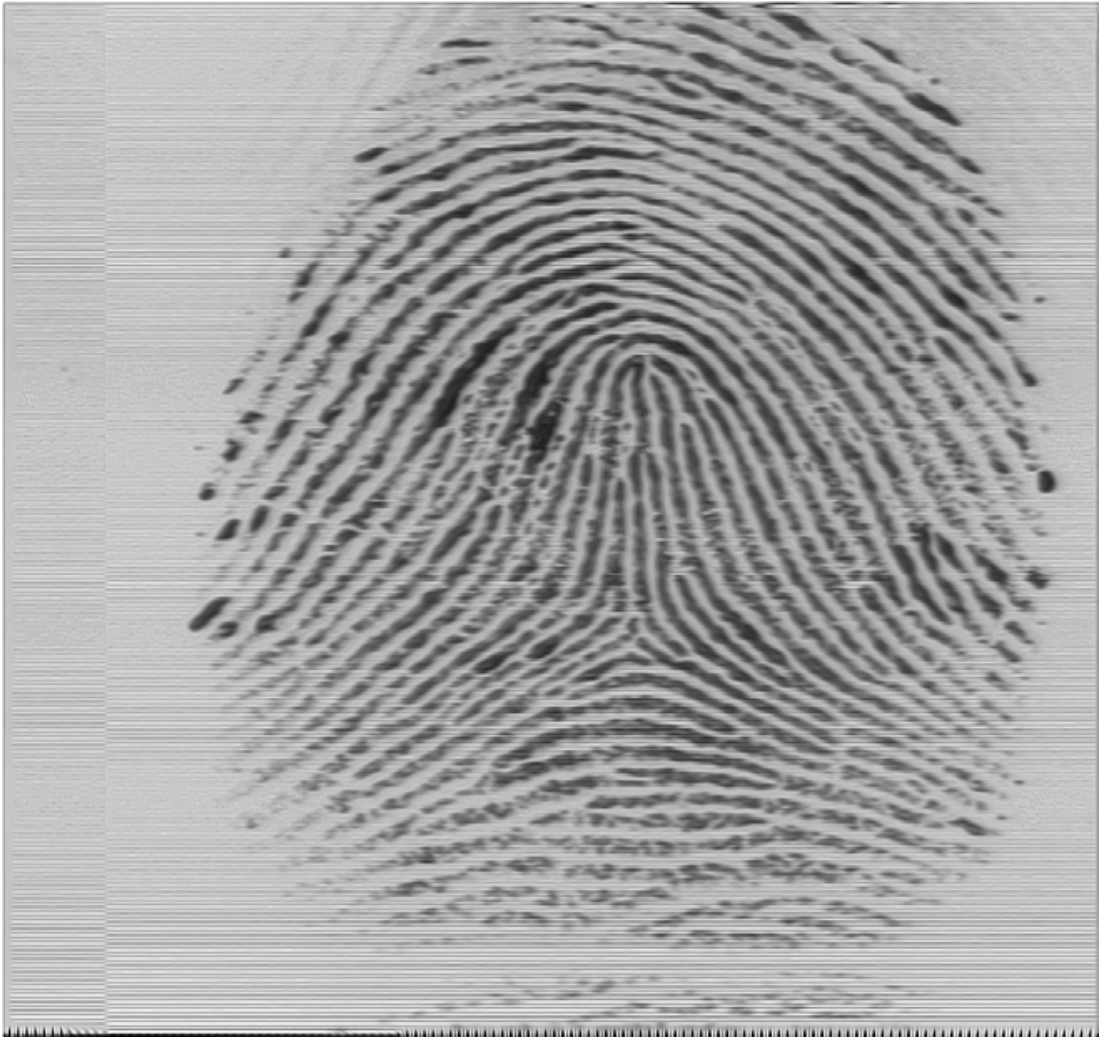

(c) Interpolated high resolution copy which will undergo restoration

Figure 30: POCS based image interpolation 


\subsection{Image Restoration}

Image restoration is a very necessary and important module in the whole super resolution process. Also it should be noted that this is the module which uses the nature of the image very effectively to enhance it. The more the number of restoration algorithms, the better the image will be. As described earlier, we have used an amalgam of three different algorithms. Initially let us look into the results of individual algorithms first and then we will present the final super resolution results.

\subsubsection{Blind Deconvolution}

In this as said earlier the main aim is to deblurr the image without any knowledge of the blurring kernel as can be seen in the following results.

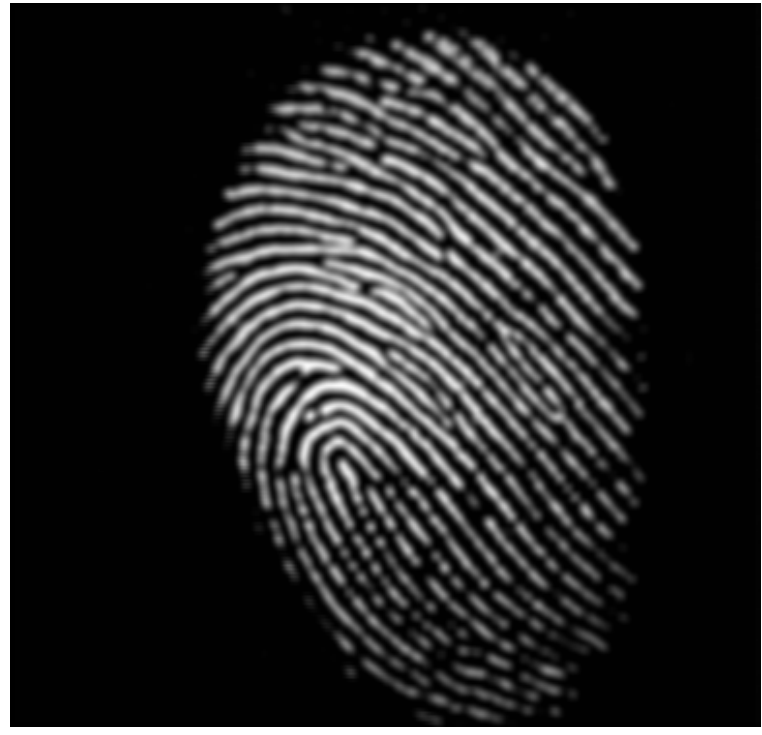

(a) Blurred image

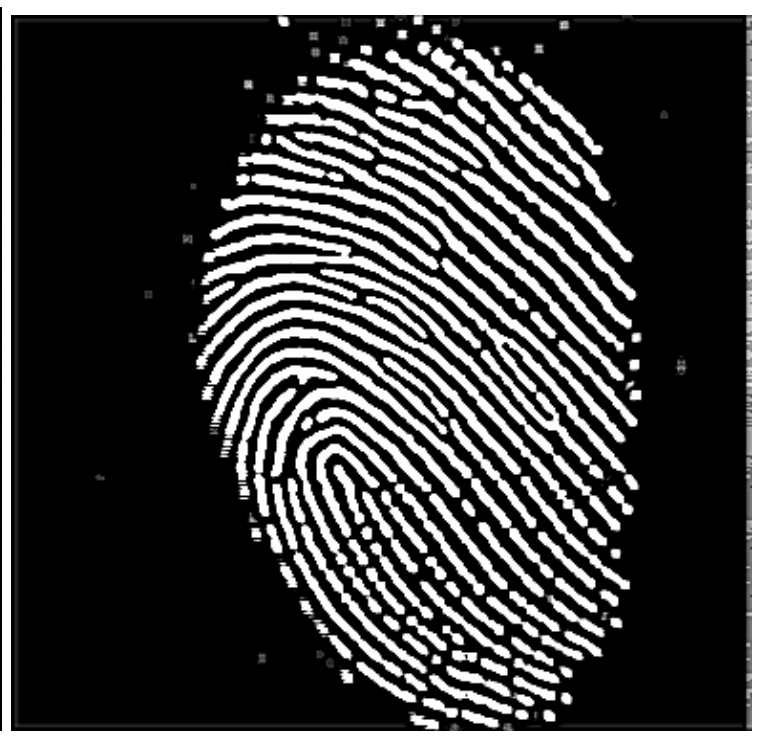

(b) image after restoration

Figure 31: Blind Deconvolution Restoration 


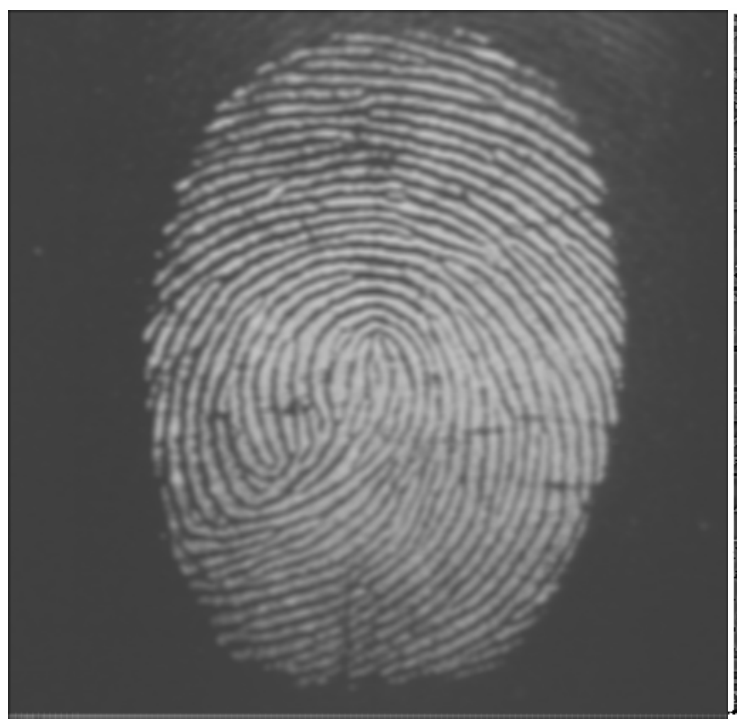

(a) Blurred image

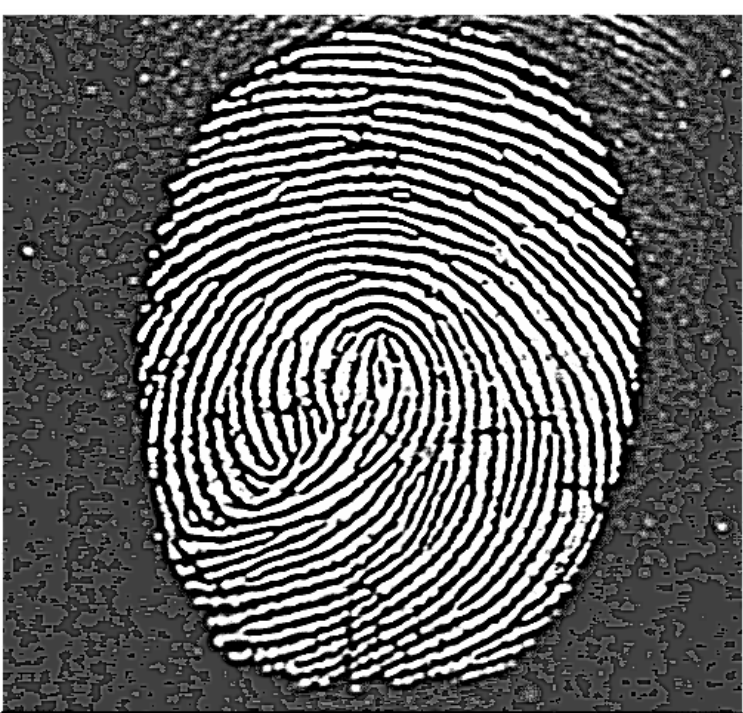

(b) image after restoration

Figure 32: Blind Deconvolution Restoration

\subsubsection{Anisotropic Coherence Enhancement}

The main aim here is the smoothening of the fingerprint along the ridge direction and not across it as is seen in the following results.

\section{A Whorl}
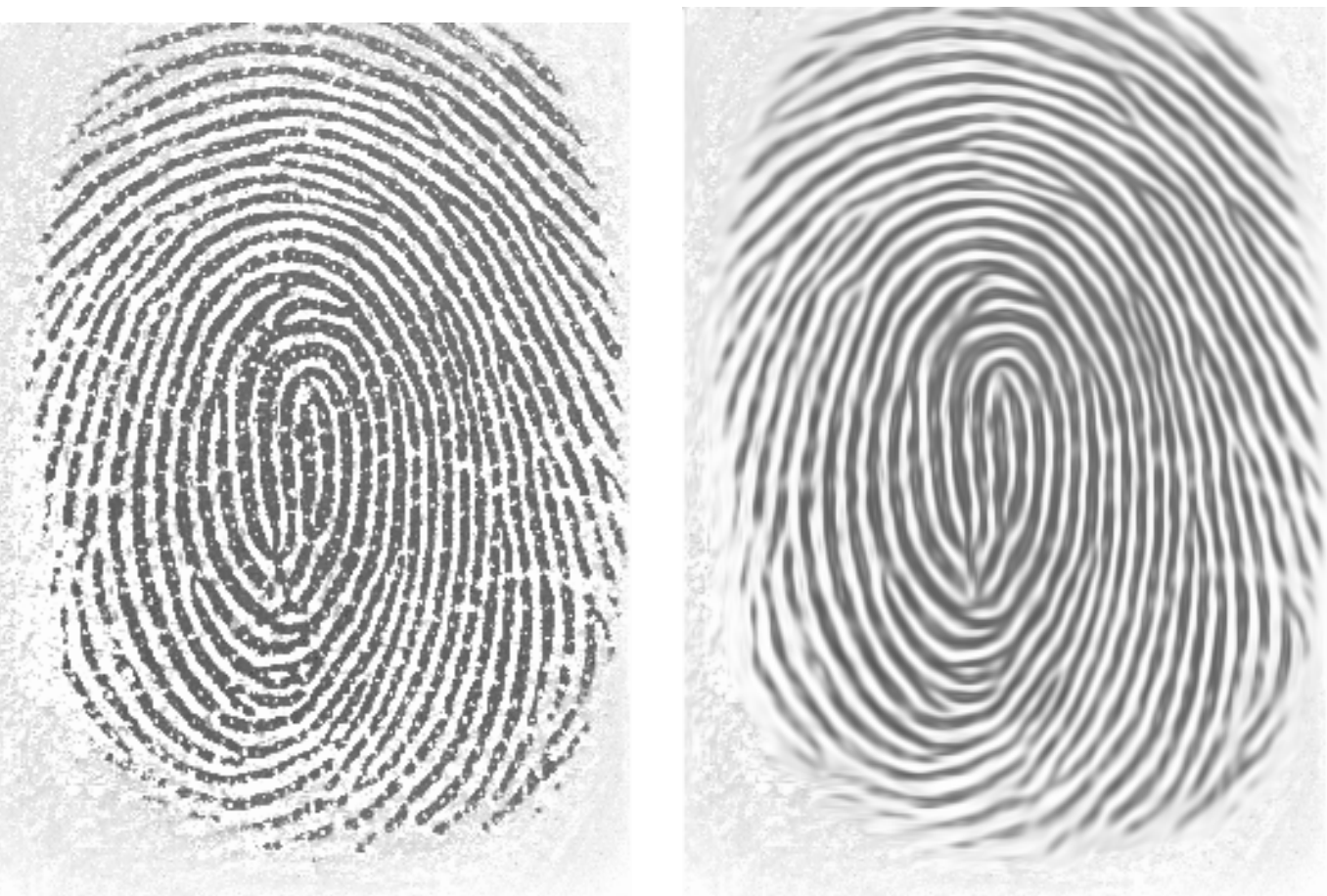

(a) Original Image

(b) Image after Coherence Enhancing Anisotropic Diffusion

Figure 33: Coherence Enhancement 
2. A Loop
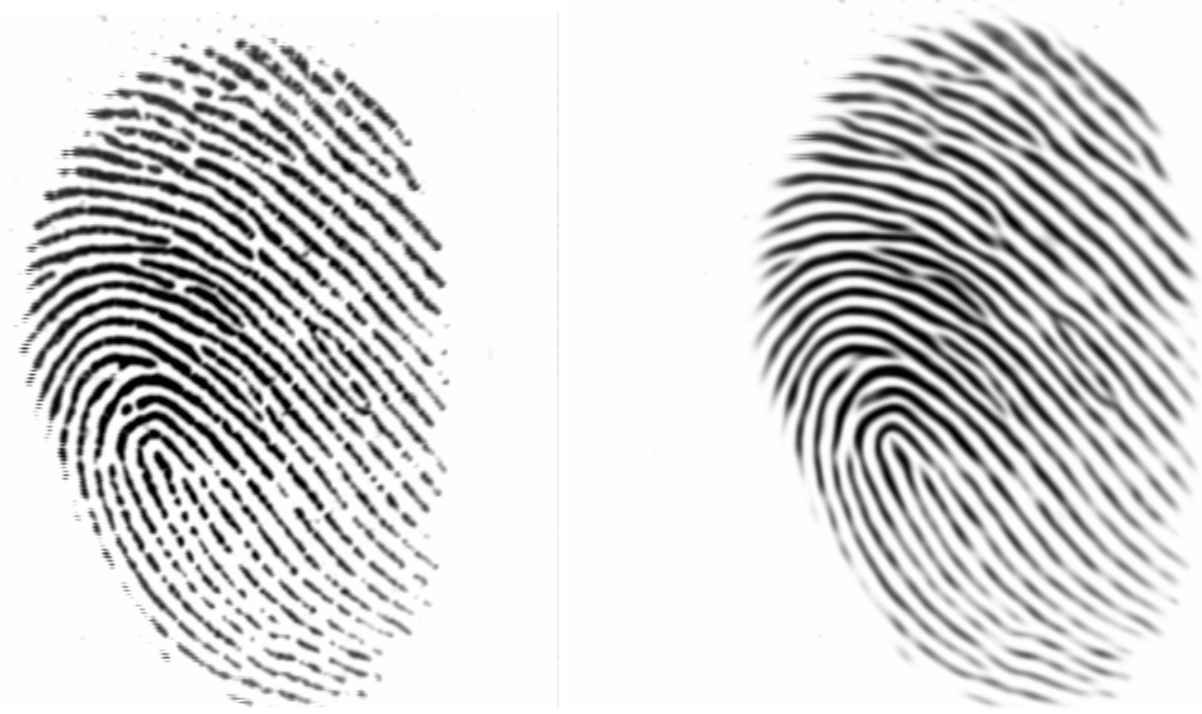

(a) Original Image

(b) Image after Coherence Enhancing Anisotropic Diffusion

Figure 34: Coherence Enhancement

\subsubsection{Image Inpainting}

The objective of image inpainting is to fill up the holes or scars in the fingerprint and thus adds texture information into the fingerprint.

One drawback the image inpainting currently has is that it should have a simple topology. Thus inpainting is not suitable for fingerprints. But when used in conjunction with other algorithms, they give very good results.

The mediocre results for image inpainting can be seen below. 


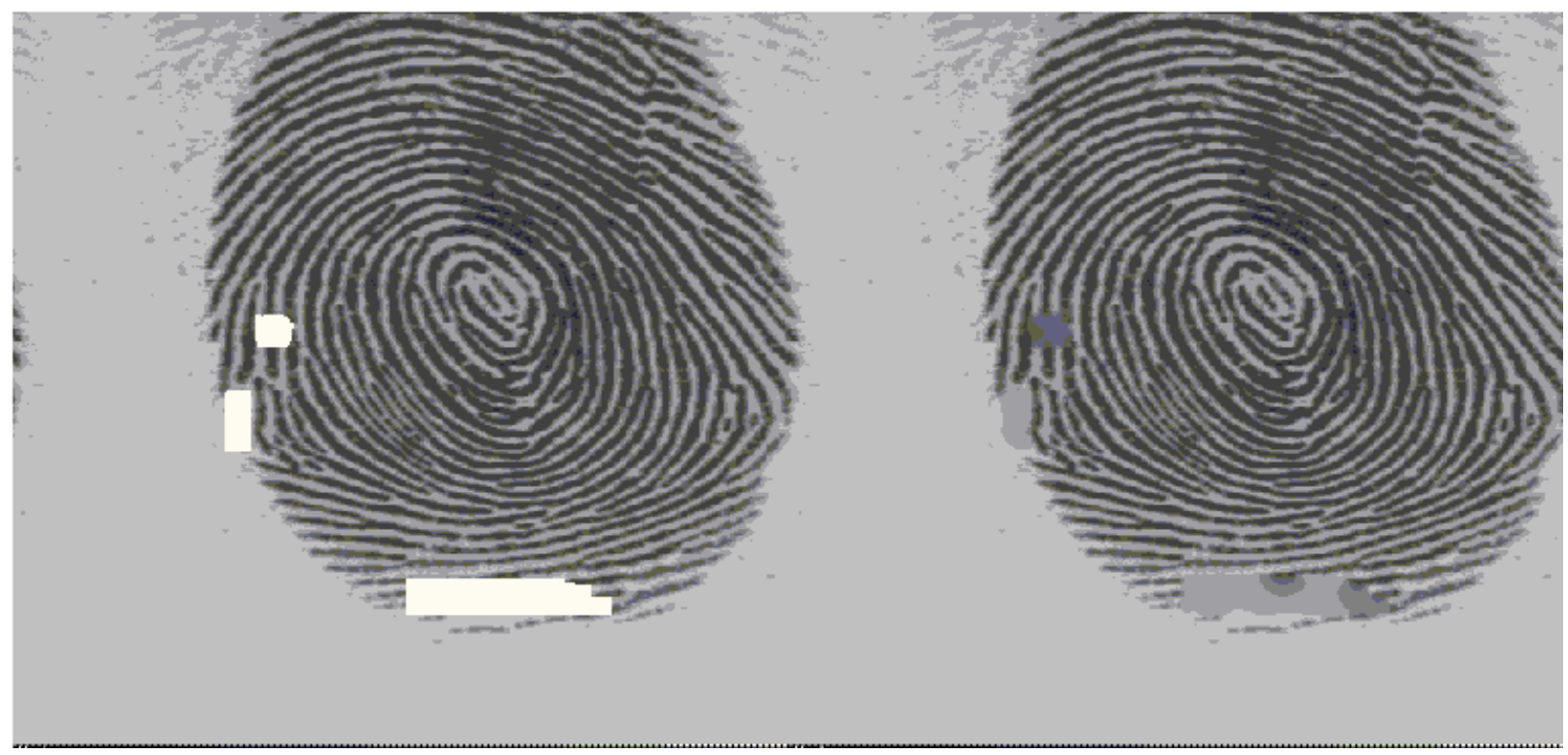

Figure 35: Image inpainting

\subsection{Super Resolution}

For the final super resolution process, we have come up with three methods of presenting the results. In the next sub section we will describe the three problem scenarios and will present the results.

1. Start with multiple copies of the same fingerprint. Some noise (blurring, scratches etc) are added to the image. Now the algorithm increases the resolution using the multiple copies and constructs a high resolution image. This high resolution image then undergoes the enhancement module to give a final image. The improvement in the quality of the image can be seen immediately. These images are also compared with other interpolation methods. 

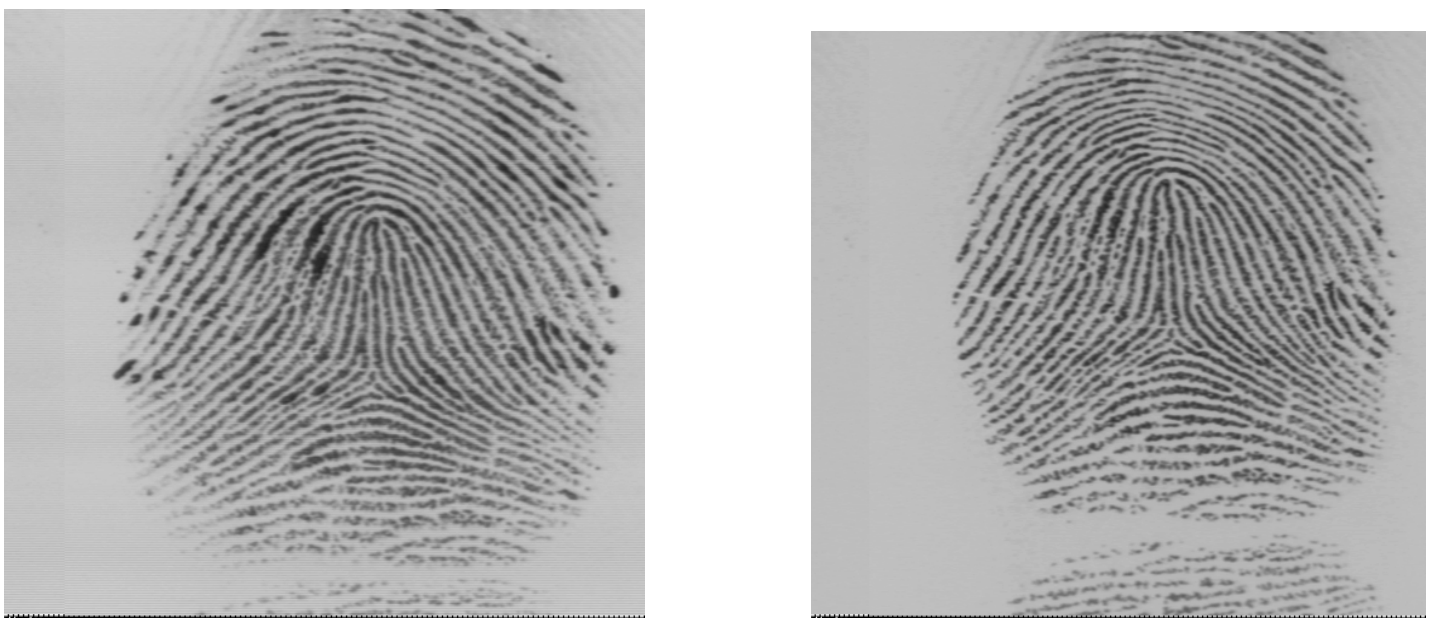

(a) Reference copy of the fingerprint (b) additional copy for resolution enhancement

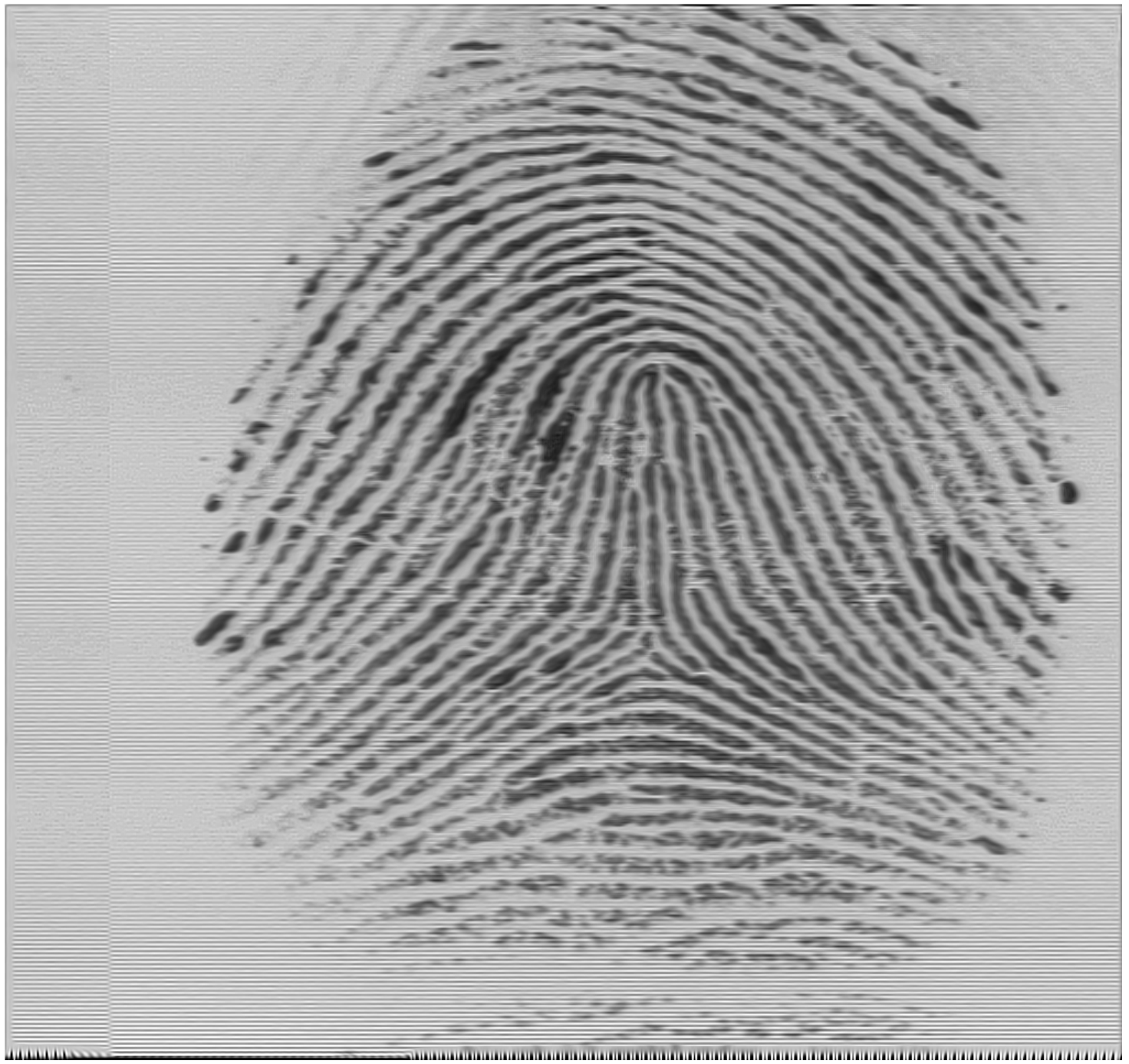

(c) Final enhanced fingerprint image

Figure 36: Super Resolution process for case (i) 


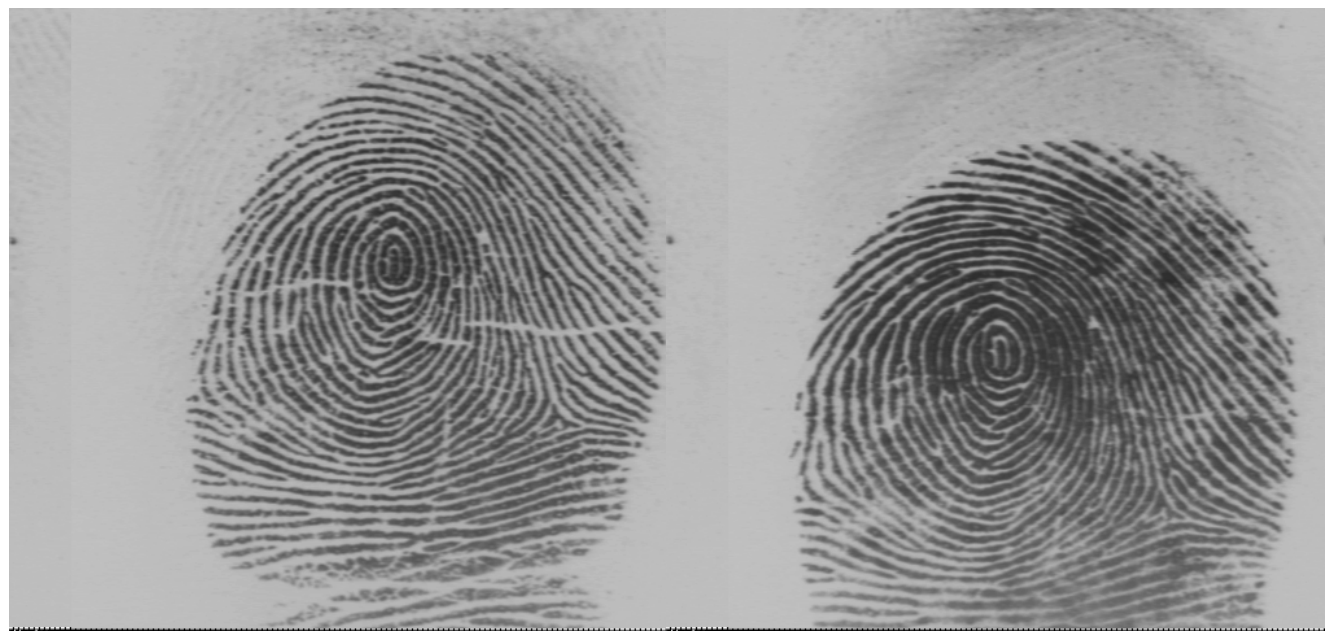

(a) Reference copy of the fingerprint (b) additional copy for resolution enhancement

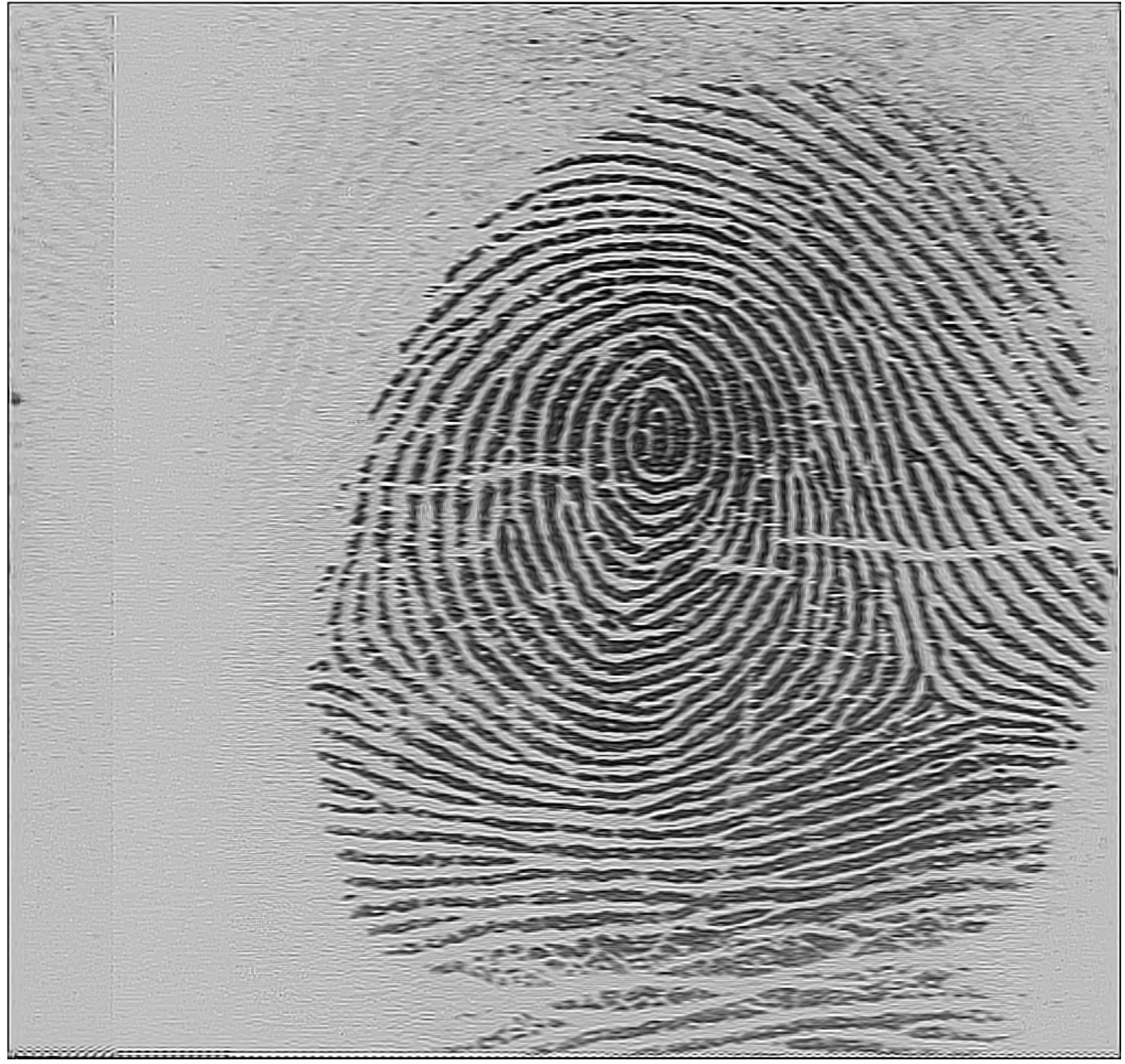

(c) Final enhanced fingerprint image

Figure 37: Super Resolution process for case (i) 

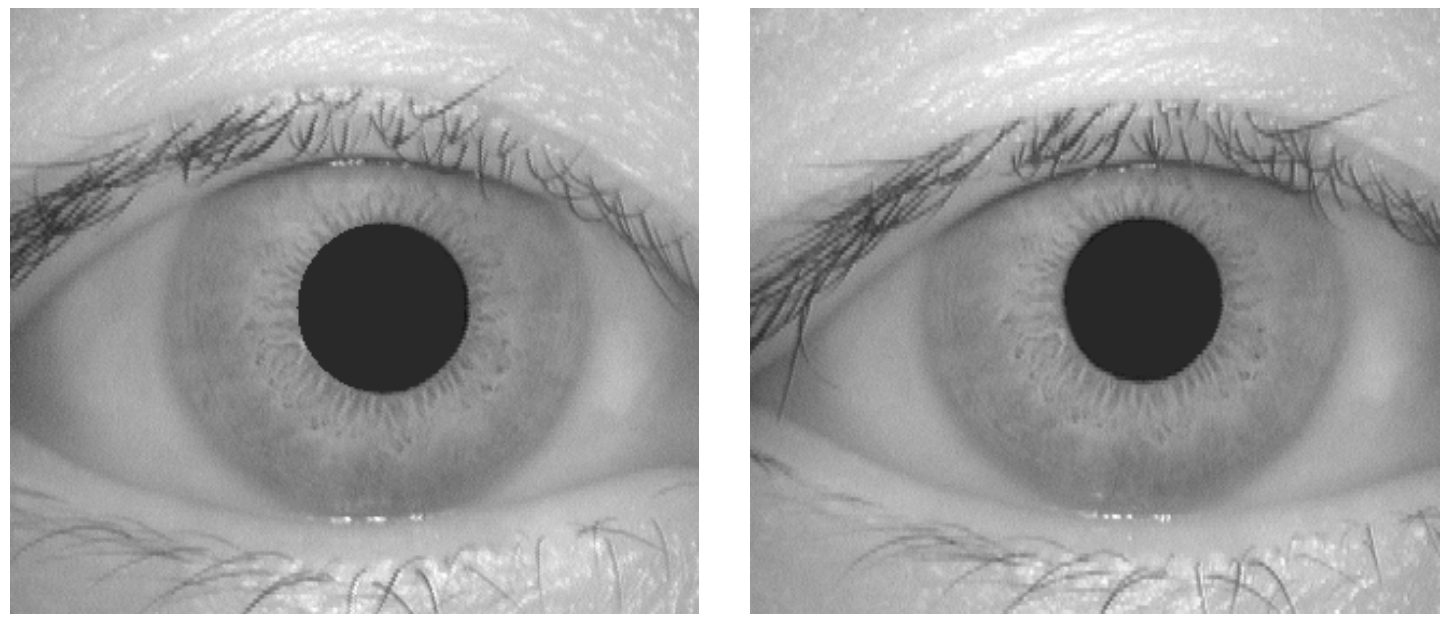

(a) Reference copy of the iris

(b) additional copy for resolution enhancement

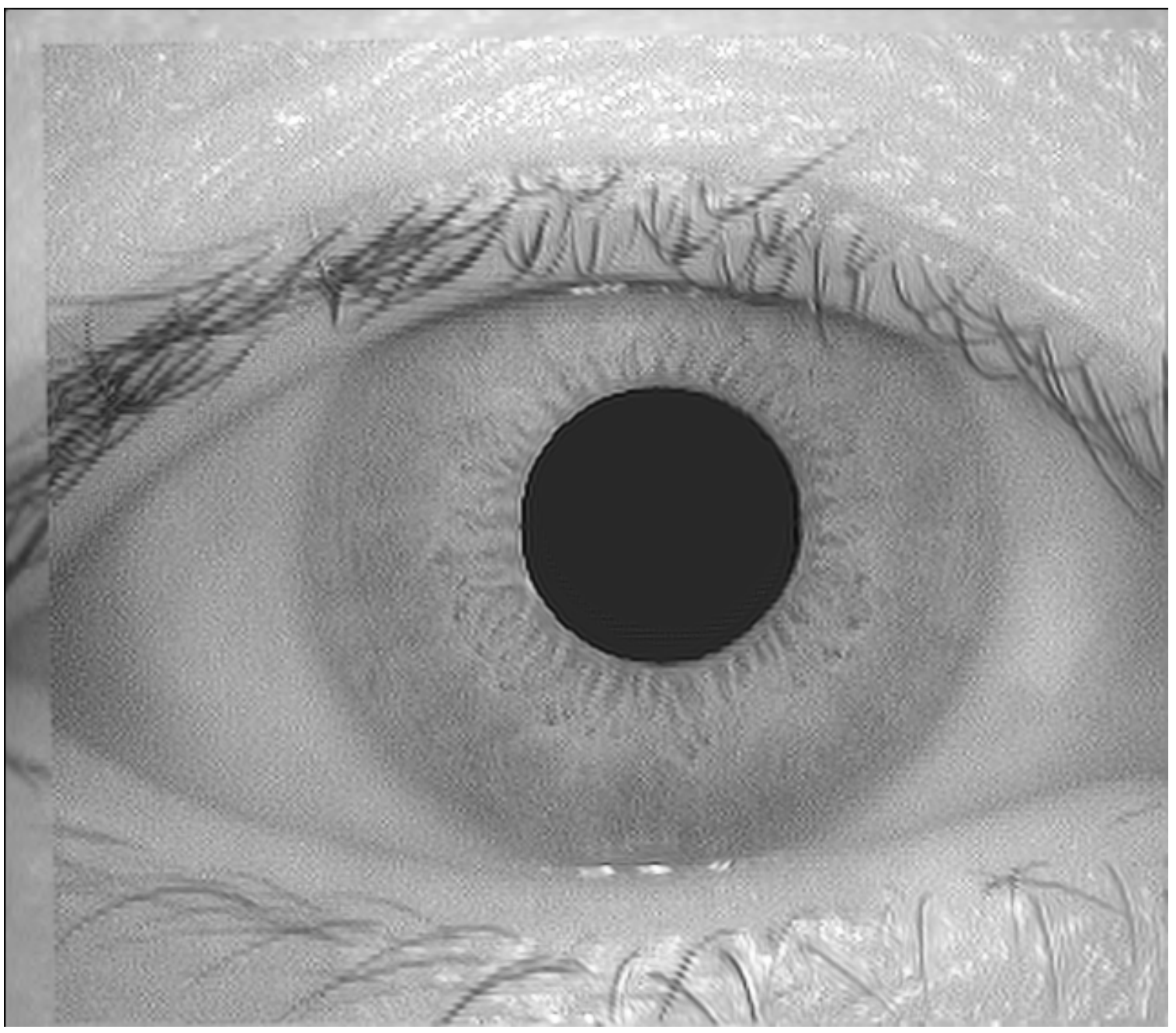

(c) Final enhanced iris image

Figure 38: Super Resolution process for case (i) 
2. Start with a high resolution image as before. But here we consider a noiseless scenario and do not add any external noise. The image is down sampled by a factor of 4 with some affine transformations. Create two or more such low resolution copies. Thus now we have a scenario where the multiple low resolution noisy copies are given to the algorithm. We use the super resolution algorithm to reconstruct the high resolution image and compare the mean square error with other interpolation methods such as the bilinear and the bicubic interpolation.
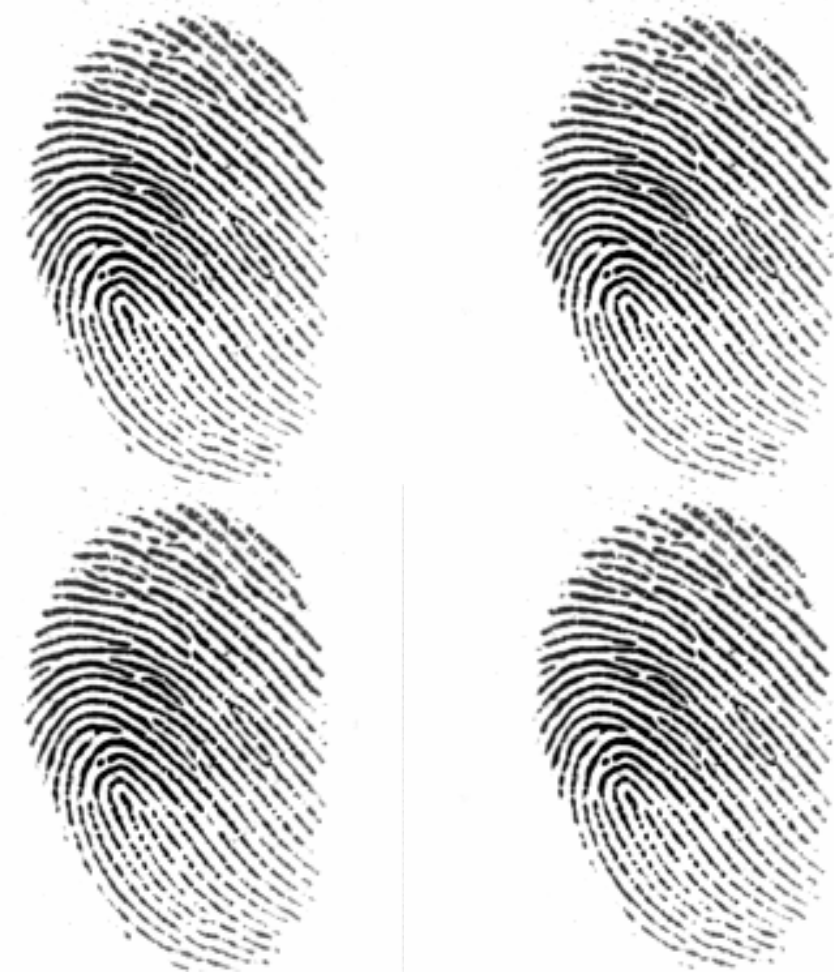

Figure 39: The four copies of the fingerprint which are used to construct the high resolution image

The following comparisons is the comparison of the mean square error when the images are interpolated by the proposed method, the bilinear interpolation an the bicubic interpolation 


\begin{tabular}{|c|c|c|c|c|}
\hline & Fingerprint & $\begin{array}{c}\text { Bilinear } \\
\text { Interpolation }\end{array}$ & $\begin{array}{c}\text { Bicubic } \\
\text { Interpolation }\end{array}$ & $\begin{array}{c}\text { Proposed Super } \\
\text { Resolution }\end{array}$ \\
\hline Mean \\
Square Error
\end{tabular}

Table 3: Comparison of mean square errors for fingerprint image
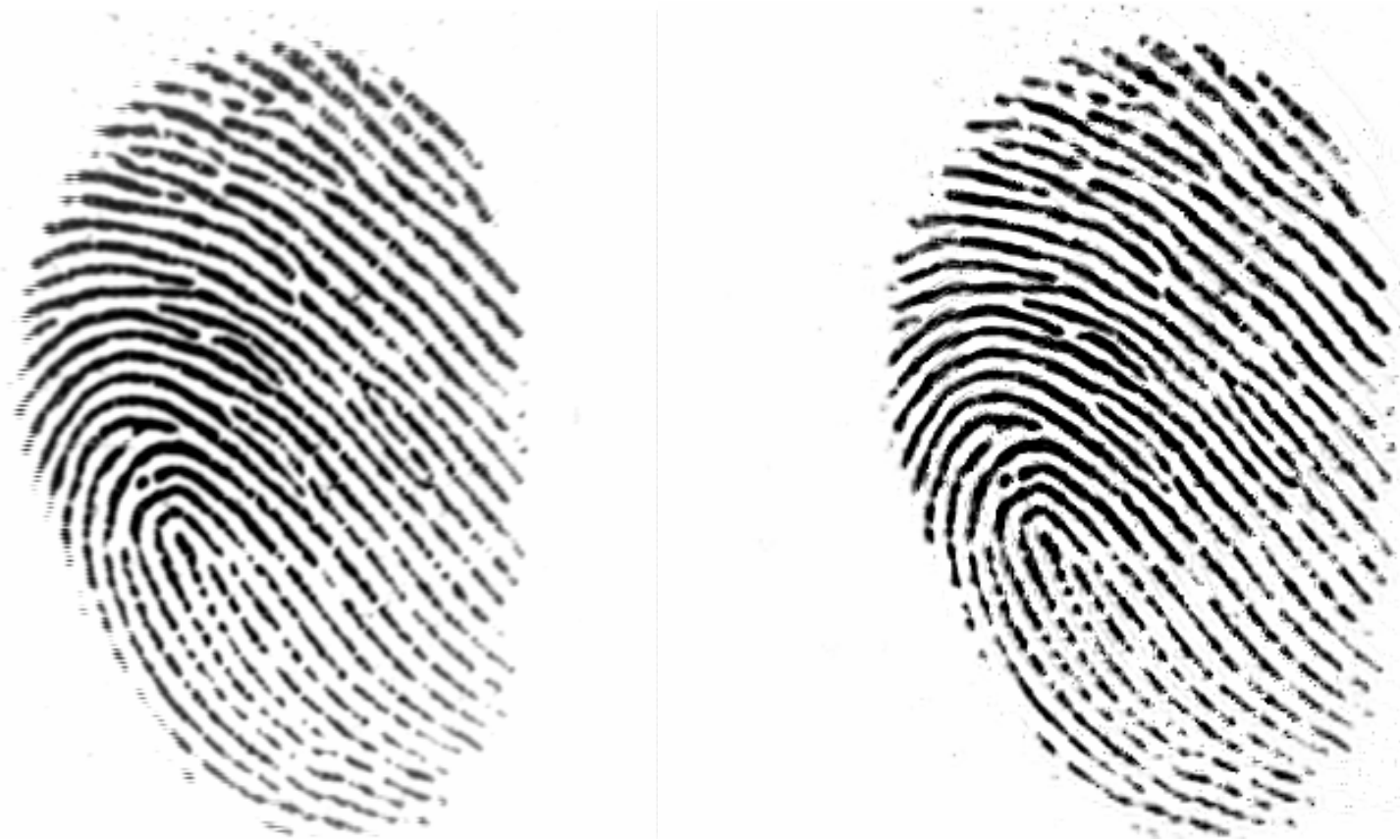

(a) Original image from which the above copies were generated (b) Reconstructed Super Resolution image

Figure 40: Visual comparison between the original image and the reconstructed image from low resolution copies 


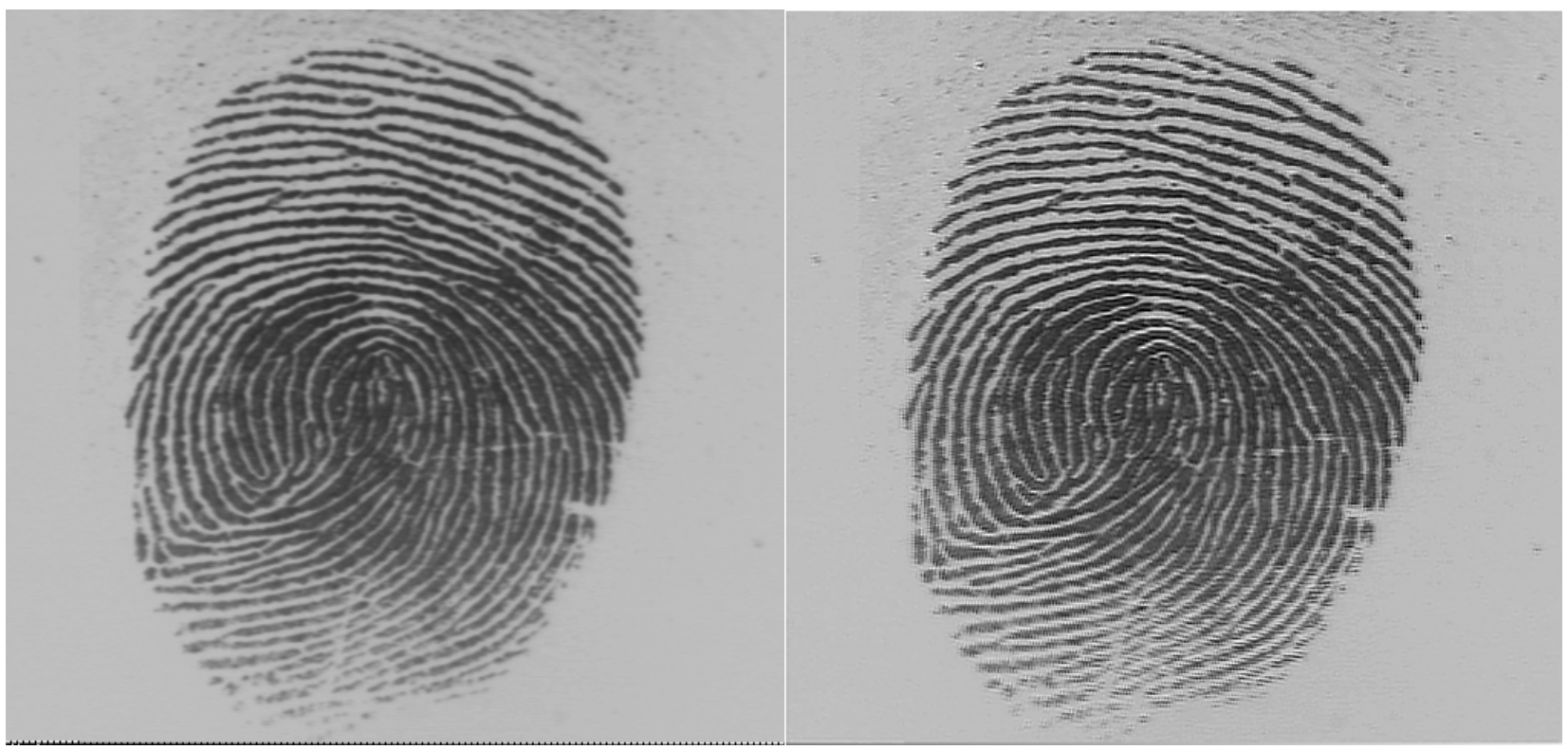

(a) Original image from which the above copies were generated (b) Reconstructed Super Resolution image

Figure 41: Visual comparison between the original image and the reconstructed image from low resolution copies

3. This problem statement is a promising direction to the application of using pores on the fingerprint for the recognition algorithms. We start with multiple copies of the same finger over a period of time to construct the high resolution image. By this we plan to locate the pores on the fingerprint which may not be available in the earlier fingerprints. Thus instead of investing in high quality sensors which are expensive and capture high resolution images, we use the normal of-the-shelf sensors to construct the same quality image which the high quality sensors give. Here we restrain the use of the coherence based enhancement technique and the image inpainting technique as they tend to blur the image and fill up the holes which may erase the pores. Thus we use the super resolution algorithm with the blind deconvolution algorithm for this purpose. 


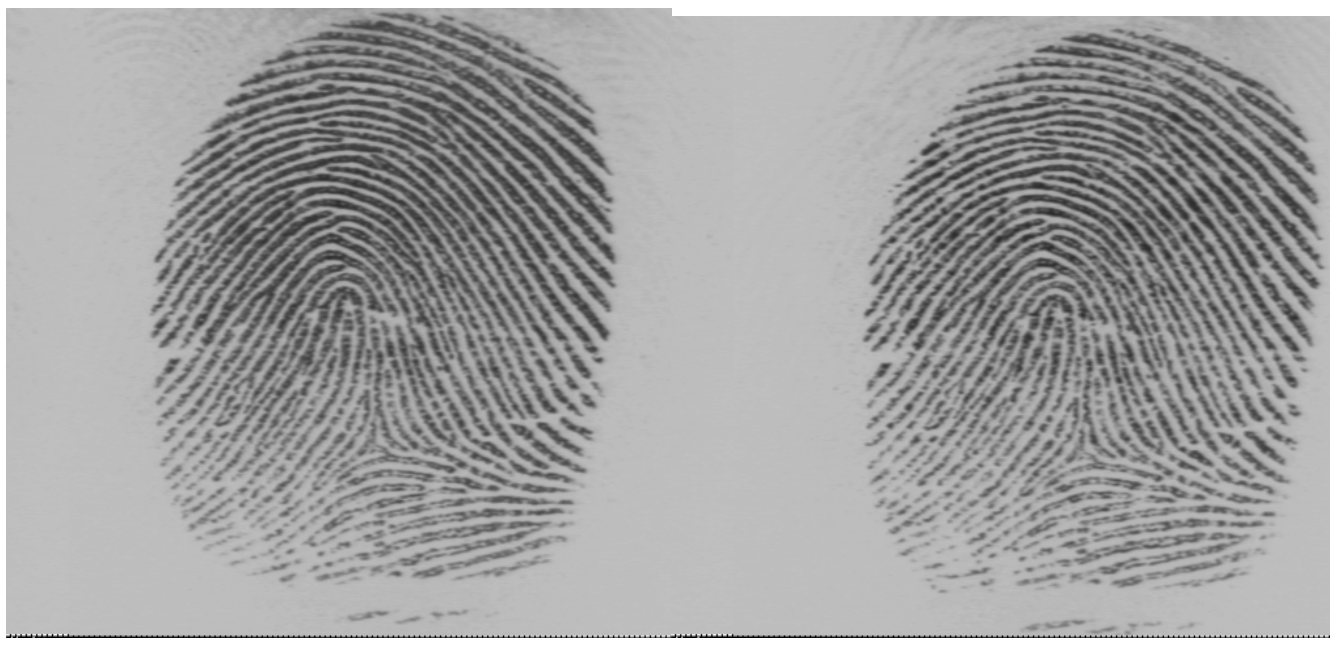

(a) Reference copy of the fingerprint (b) additional copy for pore detection

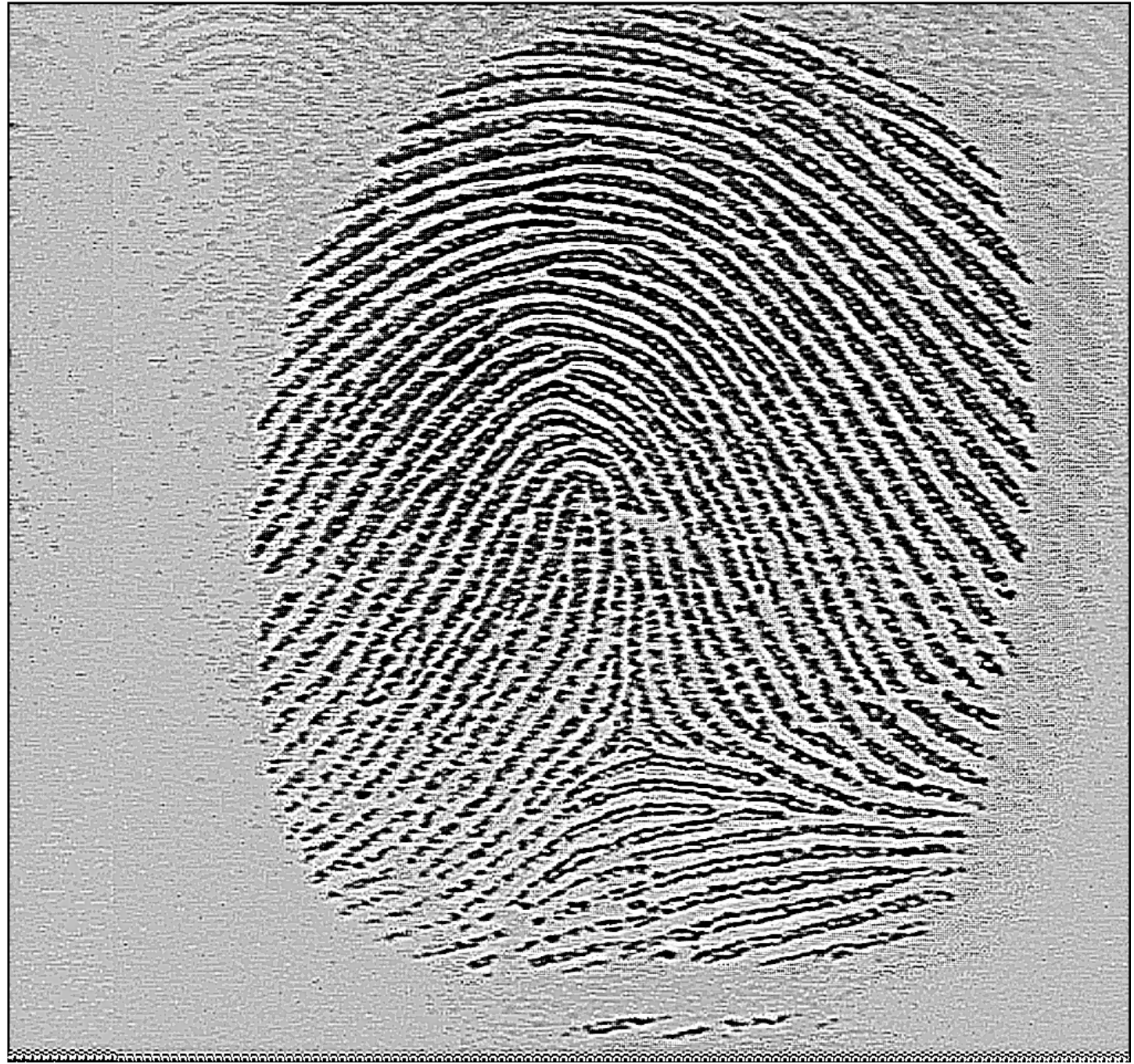

(c) The pores on the fingerprint are now visible which can be extracted.

Figure 42: Resolution improvement for detecting pores 


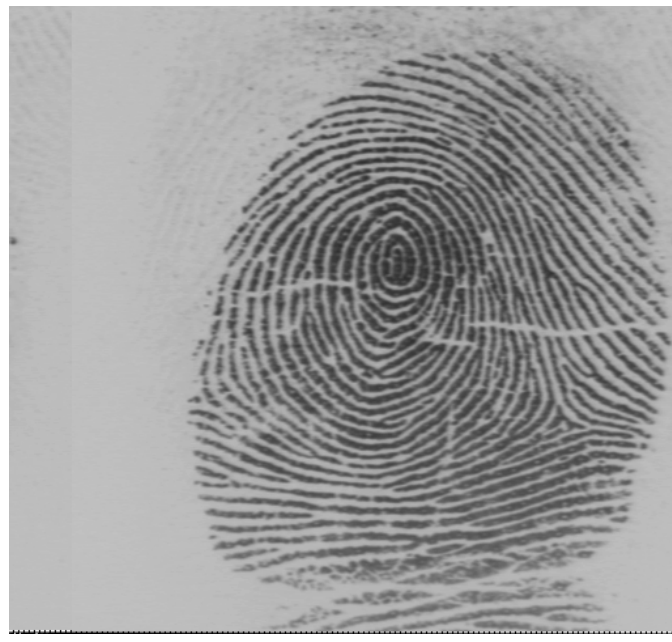

(a) Reference copy of the fingerprint

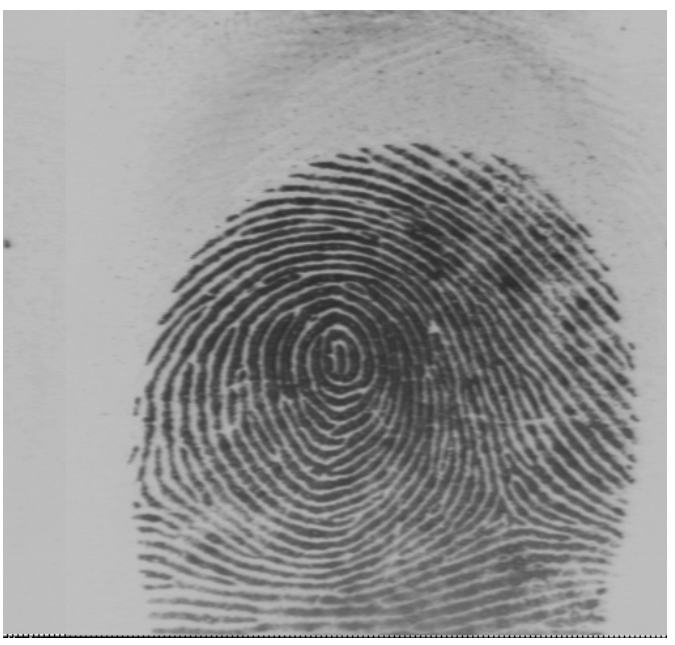

(b) additional copy for pore detection

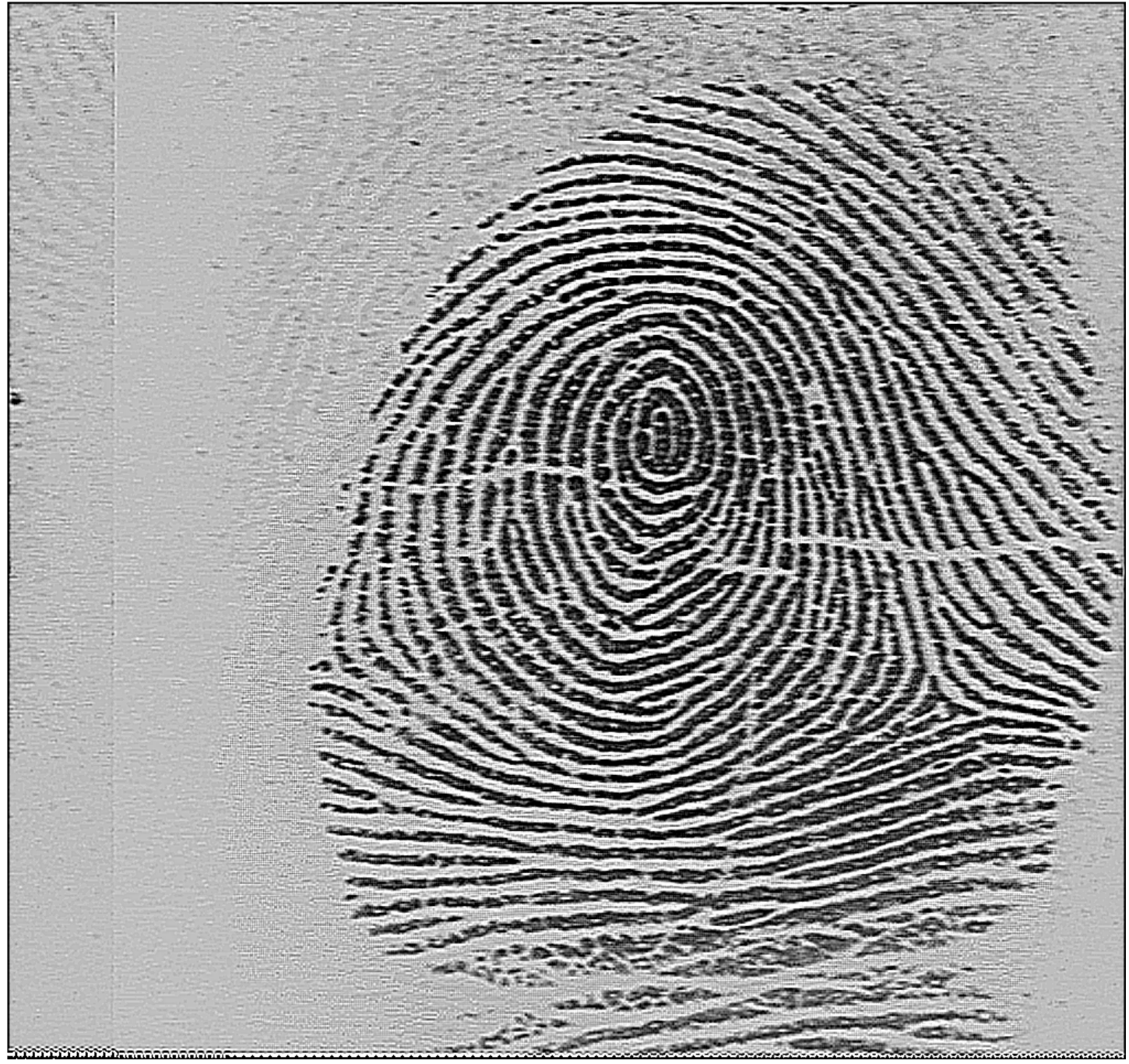

(c) The pores on the fingerprint are now visible which can be extracted.

Figure 43: Resolution improvement for detecting pores 


\section{Chapter 6}

\section{Conclusion}

With the advancement of Biometrics, more and more modalities of measuring the human subject are emerging. Out of these, considering a single biometric application, face, iris and fingerprint recognition are on the forefront. Currently, many researchers are developing many methods to achieve a $100 \%$ recognition rate. An error rate of even $1 \%$ maybe considered as a bad system considering the application. A simple example of this is the application of deploying a biometric verification system at some of the busiest airports in the country. Considering the number of people using the system everyday, an error rate of just $1 \%$ may prove to be disastrous.

One important factor in the development of the perfect system is the quality of the input images. If the input to the recognition module is of good quality, it will definitely aid in the increase of the recognition rate. Recently the need has been felt of enhancing the captured images from the biometric sensor before it is fed to the recognition algorithm. Applications such as face recognition have expressed the need of obtaining a high resolution image as its input. The reason behind this is the fact that the camera sensors are placed at a large distance from the subjects and the face image captured by them may not be enough to facilitate reliable recognition.

Another application that has come to the forefront is the use of pores on the fingerprint to aid in the minutiae matching scheme already developed. The traditional sensors do not have the resolution to capture these pores and thus considerable effort has been made to develop sensors which can capture these high resolution images. The only drawback in this is that the sensors are very expensive. We propose an economical way of solving this

problem. Instead of using the expensive sensors, we propose a way of using the commercial of-the-shelf sensors and obtain multiple images of the same fingerprint. We 
then enhance the resolution of these images which shows the placement of pores on the fingerprint.

The whole process of super resolution can be divided into three main parts. The first part is the image alignment which calculates the affine parameters between a reference image and the other available copies of the same subject. This is useful in mapping the information available in the multiple copies on the high resolution grid. We have chosen to use the phase correlation method for this purpose. The phase correlation method has the advantage of speed and simplicity.

We propose two different spaces for the estimation of the rotational parameters and the translational parameters. For the translational parameters, we directly convert the images into the frequency domain and then conduct the phase correlation process. The estimation of rotational parameters has an intermediate step of first converting the images into the $\log$ polar domain and then using the phase correlation method in this domain. In both the cases, we estimate the parameters in the sub pixel domain as that is useful to map the parameters on the high resolution grid.

The second important process in the super resolution problem is the interpolation step. In this process the available copies are mapped onto the required high resolution grid. As this is a continuous process, some kind of approximation is required for mapping the grayscale values onto the pixel domain. This is done by an iterative process of alternating projections on two or more convex sets defined by certain constraints as seen in the theory. This POCS method of image interpolation guarantees a convergence of the solution and the solution obtained is the best estimate available in the high resolution sub space. This image though is a raw image as it may have undergone certain blurring and may be missing some information.

This is where the image restoration process comes into the picture. There are many ways of restoring an image, but we have chosen three different ways of doing this. The first method is the iterative blind deconvolution method which is similar to the standard 
inverse filtering process, but the major difference is that unlike the case of inverse filtering, we do not assume any knowledge of the blurring kernel. We start with an initial approximation of the blurring kernel and iteratively using the conjugate gradient approach stabilizes to a filter value which is the best for the particular image. This is a very practical way of filtering any image as we are never sure of the blurring kernel.

The second enhancement technique is the use of a certain partial differential equation to enhance the fingerprint coherently. The main advantage of this type of enhancement is that it smoothens the image along the coherent direction, i.e. it leaves the edges intact. The results of this type of an enhancement can be clearly seen in the result section and its impact is over whelming.

The third and the final enhancement procedure is the image inpainting which uses the information in the neighboring area to fill up any holes or discontinuities within the image. This is again a form of the PDE which zeros in on the affected part of the image from all directions and uses the information from the better area to fill up the degraded area.

Thus these three enhancement techniques applied selectively give a better quality enhanced high resolution image which can be used for the applications listed above and certain other applications on similar lines. 


\section{Chapter 7}

\section{Future Work}

Biometrics as we know is a fast and emerging field. Researchers are coming up with extensive methods of attaining the golden goal... To achieve $100 \%$ recognition rate. Until this goal has been achieved, any work contributed to the field of biometrics is always going to be a stepping stone to future works. The same is true for this work.

Though the issue of super resolution has not been addressed in this field, the sub topics which constitute this broad topic have been there for quite a long time. As stated earlier, we have merged different methodologies to construct a robust super resolution algorithm which will help in achieving the golden goal.

Saying this, there are still avenues to be explored further considering this as a starting point. We are going to list a couple of these issues in the following section.

1. We have used multiple copies of the same biometric to construct the high resolution image. Now for this we need a robust alignment or matching algorithm. Using this alignment algorithm, we estimate the affine parameters, rotation and translation to align the multiple images. One issue not addresses here is the scaling. We have assumed the fingerprints to be of the same scale. If they are not, an algorithm to estimate the scaling coefficient along with the other affine parameters is necessary. Another related issue is the fact that the fingerprints are an elastic material. This means that when they are pressed onto the sensor, there is bound to be certain non linear distortion in the captured image. Though this problem is rarely addressed, this we believe is a crucial point in calculating the registration parameters between two fingerprints of the same image. 
2. Another issue which we have stated in the thesis but have not worked extensively on is the issue of estimating the quality of the fingerprints. In the flowchart outlining the process of super resolution, we state that after the interpolation and construction of the raw image, one needs to decide which enhancement module to use depending on the quality of the obtained fingerprint. Given a fingerprint image, a human can clearly point out the areas of bad fingerprint areas and the reason for such an area. But when the system is asked to automatically detect such areas it is a problem which is still open. The fact remains that in this process, there are false triggering which may ultimately lead in the loss of valuable information. There are very few works on this concept and it won't be long before such an automatic way of detecting blurs, scars, holes etc will be developed.

These were the two problems which we have left untouched related to my work and have to be addressed at a later date. There may be others which I may have overlooked and I would thank the reader profusely to make me aware of them. 


\section{References:}

[1] R. Wagner, R. Baraniuk, R. Nowak, "Distributed Image Compression for Sensor Networks Using Correspondence Analysis and Super-resolution," IEEE International Conference on Image Processing, (In-Press, September 2003).

[2] J. Weickert, "Theoretical foundations of anisotropic diffusion in image processing," Theoretical Foundations of Computer Vision, Computing Suppl. 11, W.Kropatsch, R.Klette, and F.Solina, eds., pp. 221-236. Wien: Springer, 1996.

[2a] S. Pankanti, R. M. Bolle, and A. K. Jain, "Guest Editors' Introduction: Biometrics The Future of Identification," IEEE Computer, vol. 33, no. 2. pp. 46-49, February 2000.

[2b] A. K. Jain, Biometrics: Personal Identification in Networked Society, 15 January, 1999 Kluwer Academic Publishers

[2c] A. Ross and A. K. Jain, "Information Fusion in Biometrics", Pattern Recognition Letters, vol. 24, no. 13, pp. 2115-2125, September 2003.

[3] A. Ross, "Information Fusion in Fingerprint Authentication," Ph.D. Dissertation, Michigan State University, 2003.

[4] J. Woodward, Jr., N. Orlans and P. Higgins, Biometrics: The Ultimate Reference, Dreamtech Press, 2003.

[5] A. K. Jain, A. Ross and S. Pankanti, "A Prototype Hand Geometry-based Verification System," Proc. of 2nd International Conference on Audio- and Video-based Biometric Person Authentication (AVBPA), (Washington D.C.), pp.166-171, March 1999.

[6] A. K. Jain, A. Ross and S. Prabhakar, "Fingerprint Matching Using Minutiae and Texture Features", Proc. of IEEE International Conference on Image Processing (ICIP), (Thessaloniki, Greece), pp.282-285, Oct 2001.

[6a] "Hand Geometry," [Online] Available at: http://bias.csr.unibo.it/research/biolab/bio_tree.html, As on: 07/01/2004

[6b] R. Cutler. "Face recognition using infrared images and eigenfaces." April 1996

[7] M. Negin, T. Chmielewski, M. Salganicoff, T. Camus, U. Cahn Von Seelen, P. Venetianer, and G. Zhang, "An Iris Biometric System for Public and Personal Use," IEEE Computer, vol.33, no.2, pp.70-75, February 2000. 
[8] H. C. Lee and R. E. Gaensslen, Advances in Fingerprint Technology, CRC Press, New York, USA, 1991.

[9] L. O'Gorman, "Fingerprint verification," Biometrics- Personal Identification in Networked Society, A. K. Jain, R. Bolle, and S. Pankanti, Eds., pp. 43-64, Kluwer Academic, The Netherlands, November 1999

[10] M. Tico, V. Onnia, and P. Huosmanen, "Fingerprint image enhancement based on second directional derivative of the digital image," EURASIP Journal on Applied Signal Processing, vol.10, pp. 1135-1144, 2002.

[11] N. K. Ratha, S. Chen, and A. K. Jain, "Adaptive flow orientation-based feature extraction in fingerprint images," Pattern Recognition Journal, vol. 28, no. 11, pp. 1657-1672, 1995.

[12] A. K. Jain, L. Hong, S. Pankanti, and R. Bolle, "An identity authentication system using fingerprints," Proceedings of the IEEE, vol. 85, no. 9, pp. 1365-1388, 1997.

[13] L. Hong, Y. Wan, and A. K. Jain, "Fingerprint image enhancement: Algorithm and performance evaluation," IEEE Trans. on Pattern Analysis and Machine Intelligence, vol. 20, no. 8, pp. 777-789, 1998.

[14] B. G. Sherlock, D. M. Monro, and K. Millard, "Fingerprint enhancement by directional Fourier filtering," IEE Proceedings-Vision, Image and Signal Processing, vol. 141, no. 2, pp. 87-94, 1994.

[15] A. J. Willis and L. Myers, "A cost-effective fingerprint recognition system for use with low-quality prints and damaged fingertips," Pattern Recognition Journal, vol. 34, no. 2, pp. 255-270, 2001.

[16] R. Y. Tsai and T. S. Huang, "Multi-frame image resolution and registration," Advanced in Computer Vision and Image Processing, pp. 317-339, 1984.

[17] K. Sauer and J. Allebach, "Iterative reconstruction of band-limited images from nonuniformly spaced samples," IEEE Trans. Circuits System, vol. 34, no. 12, pp. 1497-1505, December 1987.

[18] M. Irani and S. Peleg, "Improving resolution by image registration," CVGIP: Graphic Models and Image Processing, vol. 53, no. 3, pp. 231-239, May 1991.

[19] S. Borman and R. Stevenson, "Simultaneous multi-frame map super-resolution video enhancement using spatio-temporal priors," ICIP, pp. 27-35, 1999.

[20] W.T. Freeman, E.C. Pasztor, and O.T. Carmichael, "Learning low-level vision," ICCV, pp. 1182-1189, 1999. 
[21] W.T. Freeman, R.J. Thouis, and E.C. Pasztor, "Example based super-resolution," IEEE Computer Graphics and Application, vol.22, no. 2, pp.56-65, March-April 2002.

[22] A. Hertsmann, C. E. Jocobs, N. Oliver, B. Curless, and D. H. Salesin, "Image Analogies," ACM SIGGRAPH, pp. 327-340, 2001.

[23] S. Baker and T. Kanade, "Limits on super-resolution and how to break them," IEEE Trans. PAMI, vol.24, no. 9, pp. 1167-1183, September 2002.

[24] C. Liu, H.Y. Shum, and C.S. Zhang, "A two-step approach to hallucinating faces: Global parametric model and local nonparametric model," CVPR, pp. I:193-198, 2001.

[25] J. Z. Huang, L. Ma, T. N. Tan, and Y. H. Wang, "Learning-based Enhancement Model of Iris," Proceedings of British Machine Vision Conference, pp. 153-162, Norwich, U.K., 2003.

[26] D. P. Capel and A. Zisserman, "Super-resolution enhancement of text image sequences," Proceedings of the International Conference on Pattern Recognition, vol. 1, pp. 1600, September 2000.

[26a] L. G. Brown, “A Survey of Image Registration Techniques”. ACM Comput. Surv. vol. 24, no. 4, pp. 325-376, 1992.

[27] D. D. Muresan, T. W. Parks, "Adaptively Quadratic (AQua) Image Interpolation," IEEE Trans. Image Processing, no. 5, pp. 690-698, May 2004.

[28] E. Maeland, "On the comparison of interpolation methods," IEEE Trans. Medical Imaging, vol. 7, pp. 213-217, 1988.

[29] T. M. Lehmann, C. G"onner, and K. Spitzer, "Survey: Interpolation methods in medical image processing," IEEE Trans. Medical Imaging, vol. 18, pp. 1049-1075, 1999.

[30] G. Ramponi, "Warped distance for space-variant linear image interpolation," IEEE Trans. Image Processing, vol. 8, pp. 629-639, 1999.

[31] K. Jensen and D. Anastassio, "Subpixel edge localization and the interpolation of still images," IEEE Trans. Image Processing, vol. 4, pp. 285-295, 1995.

[32] J. Allebach and P. W. Wong, "Edge-directed interpolation," IEEE Proc. ICIP, pp. 707-710, 1996.

[33] S. Mallat and S. Zhong, "Characterization of signals from multiscale edges," IEEE Trans. Pattern Analysis and Machine Intelligence, vol. 14, pp. 710-732, 1992. 
[34] S. G. Chang, Z. Cvetkovic, and M. Vetterli, "Resolution enhancement of images using wavelet transform extrema interpolation," IEEE Proc. ICASSP, pp. 2379-2382, 1995.

[35] W. K. Carey, D. B. Chuang, and S. S. Hemami, "Regularity-preserving image interpolation," IEEE Trans. Image Processing, vol. 8, pp. 1293-1297, 1999.

[36] W. T. Freeman, T. R. Jones, and E. C. Pasztor, "Example-based super-resolution," MERL - Mitsubishi Electronic Research Laboratory, Tech. Rep., 2001.

[37] S. Baker and T. Kanade, "Limits on super-resolution and how to break them," IEEE Trans. On Pattern Analysis and Machine Intelligence, vol. 24, pp. 1167-1183, 2002.

[38] A. Hertzmann, C. E. Jacobs, N. Oliver, B. Curless, and D. H. Salesin, "Image analogies," Proc. SIGGRAPH, 2001.

[39] J.K. Romberg, H. Choi, and R. Baraniuk, "Bayesian tree-structured image modeling using wavelet-domain hidden markov models," IEEE Trans. On Image Processing, vol. 10, no. 7, pp. $1056-1068,2001$.

[40] J. K. Romberg, H. Choi, and B. R.G., "Shift-invariant denoising using waveletdomain hidden markov trees," Conference Record of the Thirty-Third Asilomar Conference on Signals, Systems, and Computers, vol. 2, pp. 1277-1281, 1999.

[41] K. Kinebuchi, D. D. Muresan, and T. W. Parks, "Image interpolation using waveletbased hidden markov trees," IEEE Proc. ICASSP, 2001.

[42] R. Schultz and R. Stevensonz, "A bayesian approach to image expansion for improved definition," IEEE Trans. On Image Processing, vol. 3, no. 3, pp. 233-242, 1994.

[43] R. Hardie, K. Barnard, and E. Armstrong, "Joint map registration and highresolution image estimation using a sequence of undersampled images," IEEE Trans. Image Processing, vol. 6, pp. 1621-1633, 1997.

[44] X. Li, "Edge directed statistical inference with applications to image processing," Ph.D. dissertation, Princeton University, Princeton, NJ, 2000.

[45] D. D. Muresan and T. W. Parks, "Adaptive, optimal-recovery image interpolation," IEEE Proc. ICASSP., 2001.

[46] G. Chen and R. J. P. de Figueiredo, "A unified approach to optimal image interpolation problems based on linear partial differential equation models," IEEE Transactions on Image Processing, vol. 2, no. 1, 1993. 
[47] W. K. Pratt, Digital Image Processing, $3^{\text {rd }}$ edition, John Wiley and Sons, New York, 2002.

[48] G.Sapiro, Geometric Partial Differential Equations and Image Analysis, Cambridge University Press, 2001.

[49] D.Gabor, "Information theory in electron microscopy," Lab. Invest. 14, pp. 801-807 1965.

[50] A.K.Jain, "Partial differential equations and finite-difference methods in image processing, part 1: image representation," J. Optimiz. Theory Appl., vol. 23, pp. 6591, 1977.

[51] J. J. Koenderink, “The structure of images,” Biol. Cyber. vol. 50, pp. 363-370, 1984.

[52] A. P. Witkin, "Scale Space Filtering," Proceedings of the International Joint Conference on Artificial Intelligence, ACM Inc. New York, pp. 1019-1021, 1983.

[53] R. A. Hummel, "Representations based on zero crossings in scale space," Proceedings of the IEEE Conference on Computer Vision and Pattern Recognition, IEEE, New York, pp. 204-209, 1986.

[54] P. Perona and J. Malik, "Scale-space and edge detection using anisotropic diffusion," IEEE Trans. Pattern Anal. Mach. Intell., vol.12, pp. 629-639, 1990.

[55] S. Osher and L. I. Rudin, "Feature-oriented image enhancement using shock filters," SIAM J. Num. Anal., vol. 27, pp. 919-940, 1990.

[56] L. Rudin, S. Osher and E. Fatemi, "Nonlinear total variation based noise removal algorithms," Proc. Modelisations Matematiques pour le Traitment d'Images, INRIA, pp.149-179, 1992.

[57] J. Serra, Image Analysis and Mathematical Morphology: Theoretical Advances, Academic, New York, vol. 2, 1988.

[58] H. Shekarforoush, M, Berthod, and J. Zerubia, "Subpixel Image Registration by Estimating the Polyphase Decomposition of Cross Power Spectrum," CVPR, pp. 532537, 1996.

[59] V. N. Dvorchenko, "Bounds on (deterministic) correlation functions with applications to registration," IEEE Trans. PAMI, vol.5, no. 2, pp. 206-213, 1983.

[60] Q. Tian and M. N. Huhns, "Algorithm for subpixel registration," CVGIP, vol. 35, pp. 220-223, 1986. 
[61] J. J. Pearson, D. C. Hines, S. Golosman and C. G. Kuglin, "Video rate image correlation processor," Proc. SPIE : applications of Digital Image Processing, pp. 197-205, 1977.

[62] C. A. Bernstein, L. N. Kanal, D. Lavin, and E. C. Olson, "A geometric approach to sub pixel registration accuracy," CVGIP, vol.40, pp. 334-360, 1987.

[63] T. S. Huang and R. Y. Tsai, Image Sequence Analysis: Motion Estimation, chapter 1, pp. 1-18, Springer Verlag, 1981.

[64] A. Papoulis, Signal Analysis, McGraw-Hill Book Company, 1977.

[65] A. N. Akansu and R. A. Haddad, Multiresolution Signal Decomposition, Academic Press Inc., 1995

[66] M. Vetterli and J. Kovacevic, Wavelets and Subband Coding, Prentice Hall, 1995.

[67] D. C. Youla and H. Webb, "Image Restoration by the Method of Convex Projections: Part 1-theory," IEEE Transactions on Medical Imaging, vol. MI-1, no. 2, pp. 81-94, Oct. 1982.

[68] P. Oskoui-Fard and H. Stark, "Tomographic image reconstruction using the theory of convex projections," IEEE Transactions on Medical Imaging, vol. 7, no. 1, pp. 4558, Mar. 1988.

[69] M. I. Sezan and H. Stark, "Tomographic Image Reconstruction from Incomplete view Data by Convex Projections and Direct Fourier Inversion," IEEE Transactions on Medical Imaging, vol. MI-3, no. 2, pp. 91-98, June 1984.

[70] H. Stark and P. Oskoui, "High-resolution image recovery from image-plane arrays, using convex projections," Journal of the Optical Society of America A, vol. 6, no. 11, pp. 1715- 1726, 1989.

[71] S. Borman and R. Stevenson, Spatial resolution enhancement of low-resolution image sequences - a comprehensive review with directions for future research, University of Notre Dame, Tech. Rep., 1998.

[72] A. M. Tekalp, M. K. Ozkan, and M. I. Sezan, "High-resolution image reconstruction from lower-resolution image sequences and space-varying image restoration," Proceedings of the IEEE International Conference on Acoustics, Speech and Signal Processing, San Francisco, CA, vol. 3, pp. 169-172, 1992.

[73] A. J. Patti, M. I. Sezan, and A. M. Tekalp, "High resolution standards conversion of low resolution video," Proceedings of the IEEE International Conference of Acoustics, Speech and Signal Processing, Detroit, MI, vol. 4, pp. 2197-2200, 1995. 
[74] A. J. Patti, M. I. Sezan, and A. M. Tekalp, "High-resolution image reconstruction from a low-resolution image sequence in the presence of time-varying motion blur," Proceedings of the IEEE International Conference on Image Processing, Austin, TX, vol. 1, pp. 343-347, 1994.

[75] H. J. Tussel and M. R. Civanlar, "Feasible Solution in Signal Restoration," IEEE Trans. Acoust., Speech, and Signal Proc., vol. ASSP-32, no.2, pp. 201-212, 1984.

[76] D. Kundur, Blind Deconvolution of Still Images using Recursive Inverse Filtering, Master's Thesis, Department of Electrical Engineering, University of Toronto, Toronto, Ontario, Canada, May 1995.

[77] M. Cannon, "Blind deconvolution of spatially invariant image blurs with phase," IEEE Trans. Acoust. , Speech, Signal Process. , vol. 24, no. 1, pp. 58-63, Feb. 1976.

[78] M. M. Chang, A. M. Tekalp, and A. T. Erdem, "Blur identification using the bispectrum," IEEE Trans. Signal Processing, vol. 39, no.10, pp. 2323-2325, Oct. 1991.

[79] R. Fabian and D. Malah, "Robust identification of motion and out-of-focus blur parameters from blurred and noisy images," CVGIP: Graphical Models and Image Processing, vol. 53, no. 5, pp. 403-412, Sept. 1991.

[80] B. Chalmond, "PSF estimation for image deblurring," CVGIP: Graphical Models and Image Processing, vol. 53, no. 4, pp. 364-372, July 1991.

[81] G. R. Ayers and J. C. Dainty, "Iterative blind deconvolution method and its applications," Optics Letters, vol. 13, no. 7, pp. 547-549, July 1988.

[82] B. L. K. Davey, R. G. Lane, and R. H. T. Bates, "Blind deconvolution of noisy complex-valued image," Optics Communications, vol. 69, no. 5, 6, pp. 353-356, Jan. 1989.

[83] J. H. Seldin and J. R. Fienup, "Iterative blind deconvolution algorithm applied to phase retrieval," J. Opt. Soc. Am. A, vol. 7, no. 3, pp. 428-433, Mar. 1990.

[84] N. Miura and N. Baba, "Extended-object reconstruction with sequential use of the iterative blind deconvolution method," Optics Communications, vol. 89, pp. 375-379, 1992.

[85] N. Miura, N. Baba, S. Isobe, M. Noguchi, and Y. Norimoto, "Binary star reconstruction with use of the blind deconvolution method," Journal of Modern Optics, vol. 39, no. 5, pp. 1137-1146, May 1992.

[86] F. Tsumuraya, N. Miura and N. Baba, "Iterative blind deconvolution method using Lucy's algorithm," Astron. Astrophys., vol. 282, no. 2, pp. 699-708, Feb. 1994. 
[87] N. Miura, K. Ohsawa and N. Baba, "Single-frame blind deconvolution by means of frame segmentation," Optics Letters, vol. 19, no. 10, pp. 695-697, May 1994.

[88] R. G. Lane, "Blind deconvolution of speckle images," J. Opt. Soc. Am. A., vol. 9, no. 9, pp. 1508-1514, Sept. 1992.

[89] B. C. McCallum, "Blind deconvolution by simulated annealing," Optics Communications, vol. 75, no. 2, pp. 101-105, Feb. 1990.

[90] R. L. Lagendijk, A. M. Tekalp, and J. Biemond, "Maximum likelihood image and blur identi_cation: a unifying approach," Optical Engineering, vol. 29, no. 5, pp. 422435, May 1990.

[91] R. L. Lagendijk, J. Biemond, and D. E. Boekee, "Identification and restoration of noisy blurred images using the expectation-maximization algorithm," IEEE Trans. Acoust. , Speech, Signal Process. , vol. 38, no. 7, July 1990.

[92] S. J. Reeves and R. M. Mersereau, "Blur identification by the method of generalized cross-validation," IEEE Trans. Image Processing, vol. 1, no. 3, pp. 301-311, July 1992.

[93] R. A. Wiggins, "Minimum entropy deconvolution," Geoexploration, vol. 16, pp. 21$35,1978$.

[94] G. Jacovitti and A. Neri, "A Bayesian approach to 2D non minimum phase AR identification," Fifth AASP Workshop on Spectrum Estimation and Modelling, pp. 7983, 1990.

[95] R. G. Lane and R. H. T. Bates, "Automatic multidimensional deconvolution," J. Opt. Soc. Am. A, vol. 4, no.1, pp. 180-188, Jan. 1987.

[96] D. C. Ghiglia, L. A. Romero, and G. A. Mastin, "Systematic approach to two dimensional blind deconvolution by zero-sheet separation," J. Opt. Soc. Am. A, vol. 10, no. 5, pp. 1024-1036, May 1993.

[97] T. J. Schulz, "Multiframe blind deconvolution of astronomical images," J. Opt. Soc. Am. A, vol. 10, no.5, pp. 1064-1073, May 1993.

[98] R. K. Ward and E. Lam, "Semi-blind restoration from differently blurred versions of an image," Proc. IEEE Int. Conf. Acoustics, Speech, Signal Processing, pp. 29492952, 1991.

[99] A. P. Petropulu and C. L. Nikias, "Blind deconvolution using signal reconstruction from partial higher order cepstral information," IEEE Trans. Signal Processing, vol. 41, no.6, pp. 2088-2095, June 1993. 
[100] J. Weickert, "Nonlinear diffusion filtering," Handbook of Computer Vision and Applications, vol. 2, pp. 423-450, B. Jahne and H. HauBecker, Eds. San Diego: Academic Press, 1999.

[101] P. Perona and J. Malik, "Scale space and edge detection using anisotropic diffusion," Proc. IEEE Comp. Soc. Workshop on Computer Vision, Miami Beach, pp. 16-22, Washington DC: IEEE Computer Society Press, Nov. 30 - Dec. 2, 1987.

[102] G. Ford, R. Estes, and H. Chen, "Scale space analysis for image sampling and interpolation," Proc. IEEE Int. Conf. Acoustics, Speech and Signal Processing, San Fransisco, vol. 3, pp. 165-168, March 23-26, 1992.

[103] J. Weickert, "Theoretical foundations of anisotropic diffusion in image processing," Theoretical Foundations of Computer Vision, Computing Suppl. 11, W.Kropatsch, R.Klette, and F.Solina, eds., pp. 221-236. Wien: Springer, 1996.

[104] Y. -L You and M. Kaveh, "Anisotropic blind image restoration," Proc. IEEE Int. Conf. Image Processing, Lausanne, vol.2, pp. 461-464, Sept. 16-19, 1996.

[105] J. Weickert, "Anisotropic diffusion filters for image processing based quality control," Proc. Seventh European Conf. on Mathematics in Industry, A. Fasano and M.Primicerio, eds., pp. 355-362, Stuttgart: Teubner, 1994.

[106] J. Weickert, "Multiscale texture enhancement," Computer Analysis of Images and Patterns, V.Hlavac and R.Sara, eds., vol. 970 of Lecture notes in Comp. Science, pp.230-237, Berlin:Springer,1995.

[107] R. Whitaker, "Geometry limited diffusion in the characterization of geometric patches in images," CVGIP: Image Understanding, vol. 57, pp.111-120, 1993.

[108] R. Whitaker, and G. Greig, "Vector-valued diffusion," Geometry-Driven Diffusion in Computer Vision, B.ter Haar Romeny, ed., pp. 93-134, Dordrecht: Kluwer, 1994.

[109] F. Catte, P. -L. Lions, J. M. Morel, and T. Coll, "Image selective smoothning and edge detection by non linear diffusion," SIAM J., Numer. Anal. 29, pp. 182-193, 1992.

[110] A.C.Kokaram, R.D.Morris, W.J.Fitzgerald, and P.J.W.Rayner, "Interpolation of missing data in image sequences," IEEE Trans. Image Process. vol.11, pp. 1509$1519,1995$.

[111] A.Hirani and T.Totsuka, "Combining frequency and spatial domain information for fast interactive image noise removal," Computer Graphics (SIGGRAPH Proceedings), ACM Inc., New York, pp. 269-276, 1996, 
[112] A.Efros and T.Leung, "Texture synthesis by non-parametric sampling," Proceedings of the IEEE International Conference on Computer Vision, IEEE, New York, pp.1033-1038, 1999.

[113] D.Heeger and J.Bergen, "Pyramid based texture analysis/synthesism," Computer Graphics (SIGGRAPH Proceedings), ACM Inc., New York, 1995

[114] E.Simoncelli and J.Portilla, "Texture characterization via joint statistics of wavelet coefficient magnitudes," Proceedings of the Fifth IEEE International Conference on Image Processing, New York, 1998.

[115] M.Nitzberg, D.Mumford, and T.Shiota, Filtering, Segmentaion, and Depth, Springer-Verlag, Berlin, 1993.

[116] S.Masnou and J.M.Morel. "Level-lines based disocclusion," Proceedings of the fifth IEEE International Conferrence on Image Processing, IEEE, New York, 1998. 